\title{
QM/MM Studies on the Photophysical Mechanism of a Truncated Octocrylene Model
}

\author{
Xue-Ping Chang ${ }^{1 *}$, Ye-Guang, Fang ${ }^{2}$, and Ganglong Cui ${ }^{2}$ \\ ${ }^{1}$ College of Chemistry and Chemical Engineering, Xinyang Normal University, Xinyang 464000, China \\ ${ }^{2}$ Key Laboratory of Theoretical and Computational Photochemistry, Ministry of Education, Chemistry College, \\ Beijing Normal University, Beijing 100875, China \\ E-mail: xuepingchang@xynu.edu.cn
}

\section{Contents}

1. Active Orbitals

2. Related Linearly Interpolated Cartesian Coordinate Paths

3. Bond Lengths and Dihedral Angles of Intersection Structures

4. Cartesian Coordinates of All Optimized Structures and LICC Structures 


\section{Active Orbitals}
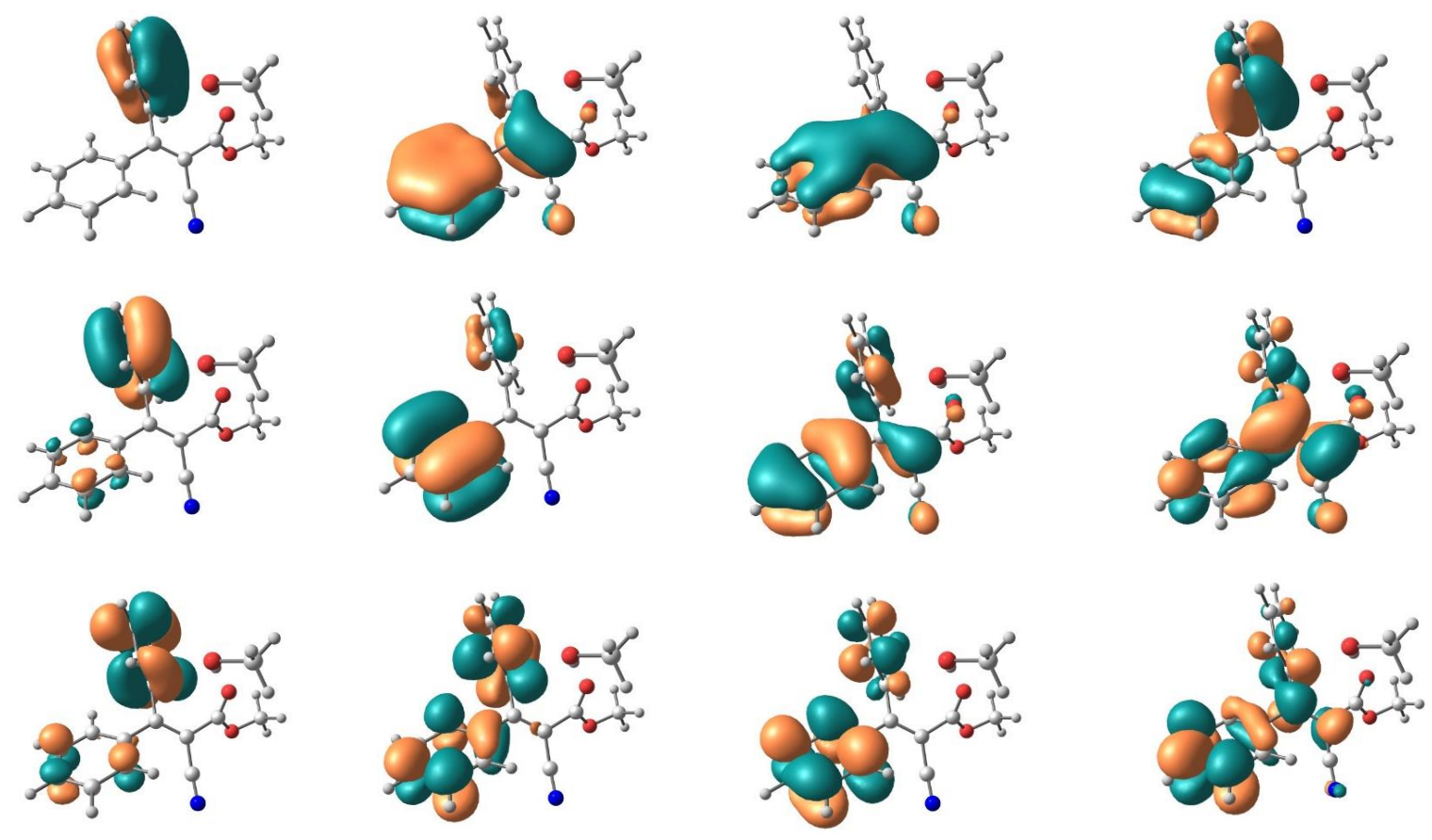

Figure S1. Active space used in QM(CASSCF)/MM and QM(CASPT2)/MM computations of the solvated MCDPA molecule in this work. 


\section{Related Linearly Interpolated Cartesian Coordinate Paths}
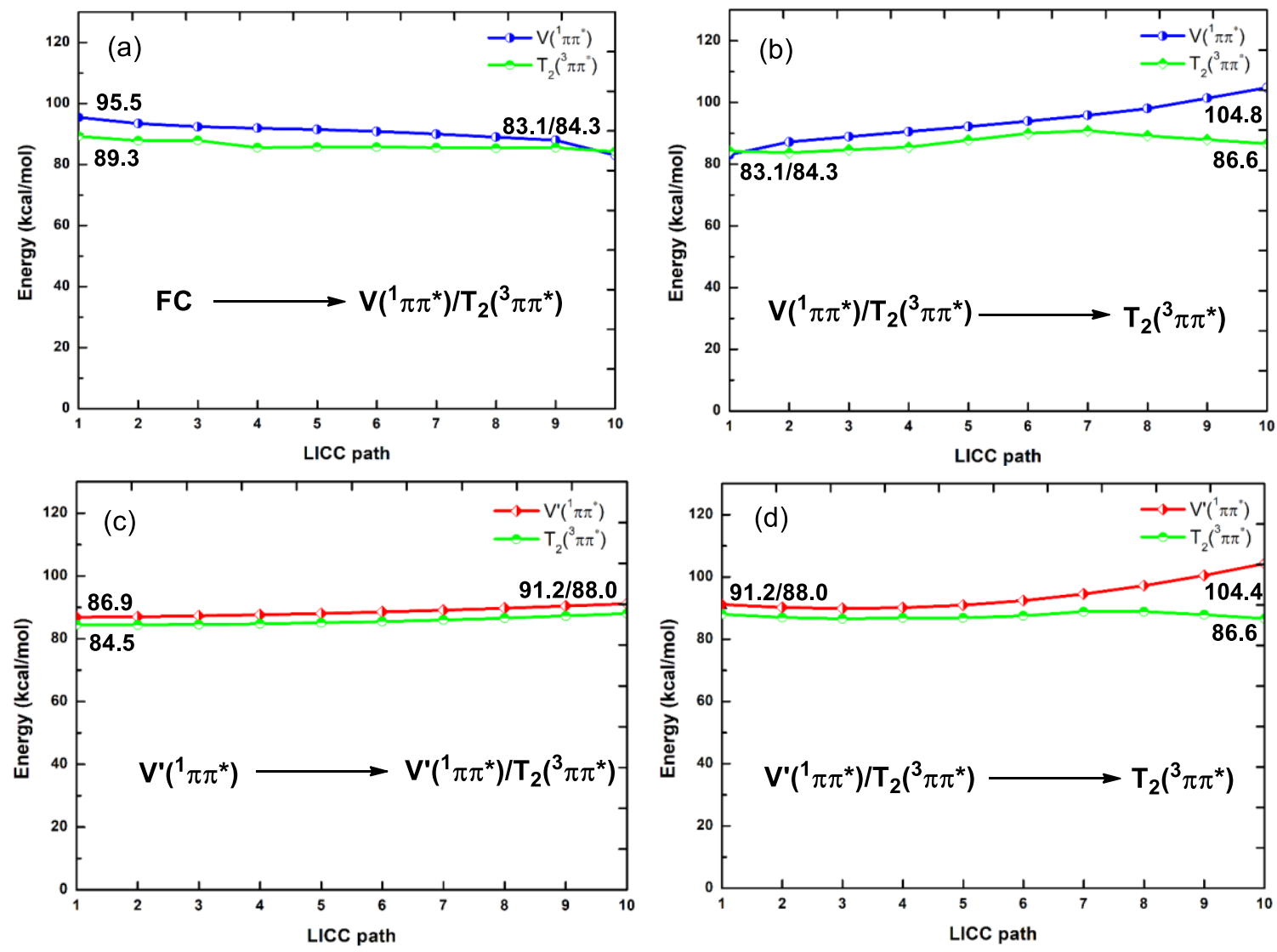

Figure S2. QM(CASPT2)/MM computed linearly interpolated Cartesian coordinate paths (LICCs, in $\mathrm{kcal} / \mathrm{mol}$ ) connecting (a) the Franck-Condon point and the $\mathrm{V}\left({ }^{1} \mathrm{~m} \mathrm{~m}^{*}\right) / \mathrm{T}_{2}\left({ }^{3} \mathrm{~m} \mathrm{~m}^{*}\right)$ crossing point; (b) the $V\left({ }^{1} \pi \pi^{*}\right) / T_{2}\left({ }^{3} \pi \pi^{*}\right)$ crossing point and the $T_{2}\left({ }^{3} \pi \pi^{*}\right)$ minimum; (c) the $V^{\prime}\left({ }^{1} \pi \pi^{*}\right)$ minimum and the $V^{\prime}\left({ }^{1} \pi \pi^{*}\right) / T_{2}\left({ }^{3} \pi \pi^{*}\right)$ crossing point; (d) the $V^{\prime}\left({ }^{1} \pi \pi^{*}\right) / T_{2}\left({ }^{3} \pi \pi^{*}\right)$ crossing point and the $T_{2}\left({ }^{3} \pi \pi^{*}\right)$ minimum. See main text for discussion. 


\section{Bond Lengths and Dihedral Angles of Intersection Structures}
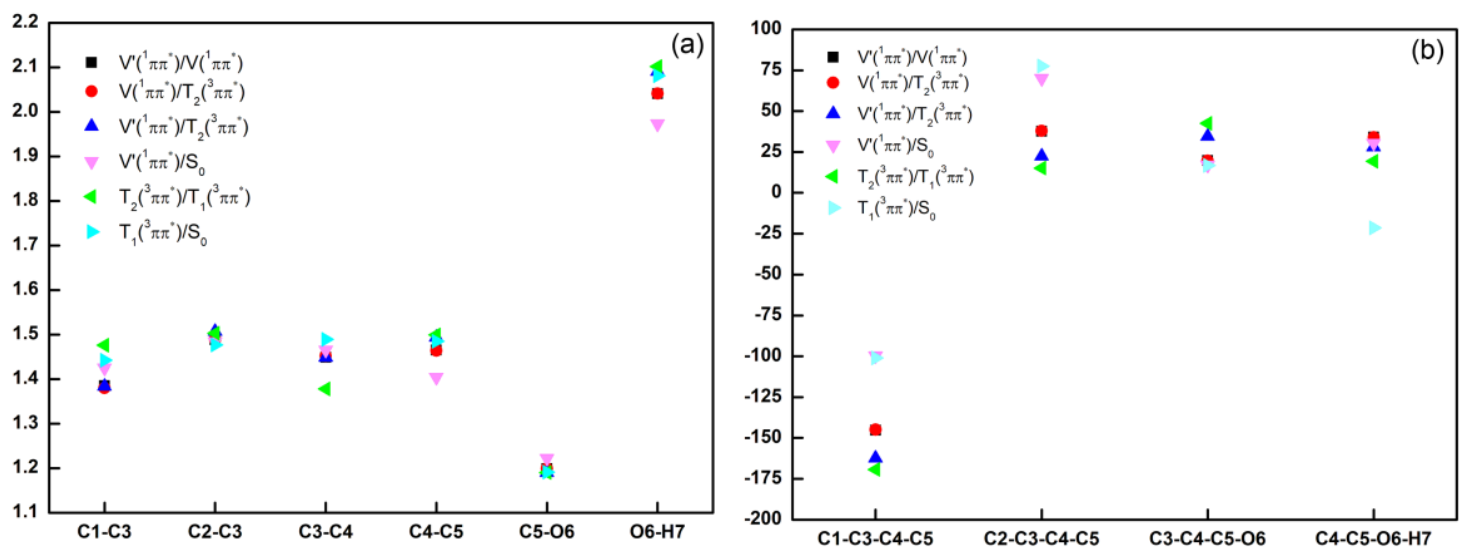

Figure S3. QM(CASSCF)/MM computed (a) bond lengths (in $\AA$ ) and (b) dihedral angles (in degree)

of intersection structures.

\section{Cartesian coordinates of All Optimized Structures and LICC Structures}

In xyz format (unit: Ångstrom)

\section{CASSCF/6-31G*}

$\begin{array}{lrrr}\text { So } & & & \\ \text { C } & -1.681776120 & -0.640966049 & 0.179419012 \\ \text { C } & -2.224746162 & -1.743826126 & 0.832706059 \\ \text { C } & -2.501392180 & 0.133228008 & -0.645971044 \\ \text { C } & -3.558730255 & -2.087840149 & 0.651287048 \\ \text { C } & -3.830556273 & -0.216633016 & -0.845859061 \\ \text { C } & -4.365533315 & -1.330585098 & -0.195822015 \\ \text { C } & 0.028662002 & 1.212328086 & 0.461213036 \\ \text { C } & -0.630727046 & 2.034235145 & 1.392501101 \\ \text { C } & 0.929961069 & 1.786946129 & -0.441234033 \\ \text { C } & -0.367966025 & 3.397525244 & 1.419194103 \\ \text { C } & 1.176309086 & 3.158129229 & -0.405834028 \\ \text { C } & 0.532062038 & 3.954936285 & 0.516519038 \\ \text { C } & -0.268981018 & -0.251633016 & 0.389242030 \\ \text { H } & -1.622261115 & -2.324934169 & 1.503474111 \\ \text { H } & -2.101863151 & 0.999638070 & -1.138553083 \\ \text { H } & -3.962444283 & -2.936192212 & 1.172639083 \\ \text { H } & -4.445592319 & 0.376940030 & -1.496623107 \\ \text { H } & -5.395673389 & -1.599208117 & -0.343334025 \\ \text { H } & -1.314926092 & 1.601517113 & 2.097254149\end{array}$




\begin{tabular}{|c|c|c|c|}
\hline $\mathrm{H}$ & 1.419948102 & 1.174222085 & -1.176900087 \\
\hline 卜 & -0.864001061 & 4.023561292 & 2.137513155 \\
\hline ト & 1.868492137 & 3.591540261 & -1.104577078 \\
\hline & 0.723771054 & 5.012981362 & 0.536940041 \\
\hline & 0.741693054 & -1.154731084 & 0.455984034 \\
\hline & 2.130642154 & -0.797395056 & 0.907369063 \\
\hline & 2.364699172 & -0.149502013 & 1.87666013 \\
\hline & 3.052242222 & -1.331833094 & 0.143662008 \\
\hline C & 4.403694319 & -1.171757082 & 0.5 \\
\hline ト & 4.672502338 & -0.125818008 & 0.55500 \\
\hline r & 4.990962360 & -1.712453123 & -0.1 \\
\hline ト & 4.546072326 & -1.585268112 & 1.53 \\
\hline C & 0.584022042 & -2.549466181 & 0.1 \\
\hline$\Lambda$ & 0.530 & -3.648670263 & -0.1 \\
\hline C & 1.110233078 & -0.338306026 & 5.2 \\
\hline 0 & 0.458 & 0.307 & \\
\hline r & 1.046195074 & 0.346939024 & 3.43 \\
\hline r & 1.3671 & -1.366339097 & \\
\hline ト & 2.019233144 & 0.179870014 & 5.52 \\
\hline & 0.43677 & -0.35 & \\
\hline \multicolumn{4}{|c|}{$V^{\prime}\left({ }^{1} \pi \pi^{*}\right)$} \\
\hline C & -1.6157 & -0.656429047 & 0.271572018 \\
\hline C & -2.103145151 & -1.966966143 & 0.642862048 \\
\hline C & -2.645629192 & 0.238961015 & 146014 \\
\hline C & -3.461291250 & -2.390037173 & 0.403339031 \\
\hline C & -3.999261287 & -0.196583014 & -0.45 \\
\hline $\mathrm{C}$ & -4.416354318 & -1.516542111 & -0.167266012 \\
\hline C & 0.020046001 & 1.216707086 & 0.4 \\
\hline C & -0.453628031 & 2.068871150 & 1.40 \\
\hline C & 0.780459055 & 1.755676125 & -0.63106204 \\
\hline C & -0.167195012 & 3.429511246 & 1.366404101 \\
\hline C & 1.072955075 & 3.118598223 & -0.657238048 \\
\hline C & 0.598750045 & 3.952057285 & 0.3300 \\
\hline C & -0.287519023 & -0.259383018 & 0.42172903 \\
\hline $\mathrm{H}$ & -1.460380106 & -2.644785191 & 1.16069108 \\
\hline $\mathrm{H}$ & -2.397847173 & 1.257658091 & -0.424549032 \\
\hline $\mathrm{H}$ & -3.732463269 & -3.387610245 & 0.68809105 \\
\hline $\mathrm{H}$ & -4.690968338 & 0.511194039 & -0.866051062 \\
\hline t & -5.417658392 & -1.840667134 & -0.36879702 \\
\hline $\mathrm{H}$ & -1.010295075 & 1.664902118 & 2.235058160 \\
\hline $\mathrm{H}$ & 1.152946085 & 1.116348080 & -1.41151310 \\
\hline $\mathrm{H}$ & -0.530240038 & 4.076690291 & 2.14412315 \\
\hline $\mathrm{H}$ & 1.675135120 & 3.514600254 & -1.45472710 \\
\hline $\mathrm{H}$ & 0.824341058 & 5.003416361 & 0.30 \\
\hline C & 0.781194056 & -1.162559082 & 0.494153034 \\
\hline C & 2.113601154 & -0.771361054 & \\
\hline
\end{tabular}




$\begin{array}{lrrr}\mathrm{O} & 2.310019165 & 0.058767007 & 1.837387130 \\ \mathrm{O} & 3.088139220 & -1.464707104 & 0.441251031 \\ \mathrm{C} & 4.405653317 & -1.205425085 & 0.887949063 \\ \mathrm{H} & 4.670853337 & -0.174054012 & 0.705752050 \\ \mathrm{H} & 5.041486362 & -1.864477137 & 0.317931025 \\ \mathrm{H} & 4.496870325 & -1.416983100 & 1.943914140 \\ \mathrm{C} & 0.679138048 & -2.503717178 & 0.005525002 \\ \mathrm{~N} & 0.588236044 & -3.570279258 & -0.384584027 \\ \mathrm{C} & 1.116611082 & -0.301590021 & 5.181102371 \\ \mathrm{O} & 0.434719031 & 0.356166023 & 4.157248298 \\ \mathrm{H} & 1.007624074 & 0.424873032 & 3.401409246 \\ \mathrm{H} & 1.388091102 & -1.318638096 & 4.904811351 \\ \mathrm{H} & 2.021349144 & 0.225804017 & 5.475578392 \\ \mathrm{H} & 0.459340034 & -0.349627027 & 6.039230433\end{array}$

$\begin{array}{lrrr}\mathrm{T}_{2}\left({ }^{3} \pi \pi^{*}\right) & & \\ \mathrm{C} & -1.709936123 & -0.669024048 & 0.194563012 \\ \mathrm{C} & -2.245596163 & -1.823858131 & 0.794847057 \\ \mathrm{C} & -2.562954185 & 0.125829010 & -0.595818043 \\ \mathrm{C} & -3.557087255 & -2.176119154 & 0.603381044 \\ \mathrm{C} & -3.887260277 & -0.246572018 & -0.802401057 \\ \mathrm{C} & -4.392184317 & -1.398926101 & -0.204146015 \\ \mathrm{C} & -0.007244003 & 1.202845086 & 0.463695036 \\ \mathrm{C} & -0.583352040 & 2.020499143 & 1.402012099 \\ \mathrm{C} & 0.903855067 & 1.775581127 & -0.524747035 \\ \mathrm{C} & -0.293794023 & 3.439093249 & 1.424739103 \\ \mathrm{C} & 1.229893087 & 3.201601230 & -0.450956030 \\ \mathrm{C} & 0.663134046 & 3.986550286 & 0.459496036 \\ \mathrm{C} & -0.310412025 & -0.266072019 & 0.415335030 \\ \mathrm{H} & -1.634149119 & -2.424327176 & 1.440008105 \\ \mathrm{H} & -2.186775159 & 1.020415072 & -1.054726078 \\ \mathrm{H} & -3.944085283 & -3.053552221 & 1.088776078 \\ \mathrm{H} & -4.519297328 & 0.360188024 & -1.424128104 \\ \mathrm{H} & -5.415843388 & -1.687483122 & -0.357314025 \\ \mathrm{H} & -1.241903088 & 1.611825116 & 2.144771154 \\ \mathrm{H} & 1.288549094 & 1.172198082 & -1.323704095 \\ \mathrm{H} & -0.765111052 & 4.077607292 & 2.143889153 \\ \mathrm{H} & 1.936347138 & 3.598796260 & -1.157033085 \\ \mathrm{H} & 0.899909066 & 5.034142361 & 0.507058037 \\ \mathrm{C} & 0.727576050 & -1.182781088 & 0.488858033 \\ \mathrm{C} & 2.103276149 & -0.803042060 & 0.944398067 \\ \mathrm{O} & 2.318508168 & -0.087221005 & 1.870472133 \\ \mathrm{O} & 3.039344220 & -1.379986102 & 0.229789018 \\ \mathrm{C} & 4.385875317 & -1.170523083 & 0.629914044 \\ \mathrm{H} & 4.632059332 & -0.120212010 & 0.580915041 \\ \mathrm{H} & 4.985402358 & -1.733428127 & -0.066780004 \\ \mathrm{H} & 4.537119324 & -1.531808113 & 1.636336119\end{array}$




\begin{tabular}{|c|c|c|c|}
\hline C & 0.586932040 & -2.563449182 & 0.102287009 \\
\hline $\mathrm{N}$ & 0.527003040 & -3.655627261 & -0.203302014 \\
\hline C & 1.107701081 & -0.304165024 & 5.234401379 \\
\hline 0 & 0.432300029 & 0.343559026 & 4.198 \\
\hline $\mathrm{H}$ & 1.003139070 & 0.388689029 & 3.441463249 \\
\hline ト & 1.366513101 & -1.329169094 & 4.976276358 \\
\hline ト & 2.018574144 & 0.218177017 & 5.518867398 \\
\hline r & 0.449913032 & -0.328692024 & 6.0925 \\
\hline \multicolumn{4}{|c|}{$\mathrm{T}_{1}\left({ }^{3} \pi \pi^{*}\right)$} \\
\hline C & -1.483897108 & -0.613726047 & 0.254237018 \\
\hline C & -1.567900112 & -1.998227141 & 0.544338040 \\
\hline C & -2.699690194 & 0.087816007 & 0.07 \\
\hline C & -2.776670201 & -2.626607191 & 0.669582049 \\
\hline$C$ & -3.924095284 & -0.560557042 & 0.20 \\
\hline $\mathrm{C}$ & -3.971391284 & -1.918546139 & 0.50 \\
\hline$C$ & -0.001006998 & 1.501887108 & 0.12 \\
\hline$C$ & -0.665273049 & 4166 & \\
\hline C & 0.936720070 & 2.106460150 & -0.7310 \\
\hline $\mathrm{C}$ & -0.413116031 & 3.686008264 & \\
\hline $\mathrm{C}$ & 1.190413088 & 3.455020246 & -0.669265046 \\
\hline$c$ & 07040 & 4.261014305 & 0.24 \\
\hline C & -0.209220015 & 0.040327005 & 0.090774007 \\
\hline $\mathrm{H}$ & -0.668367048 & -2.569154184 & 0.68 \\
\hline $\mathrm{H}$ & -2.680402191 & 1.125722080 & -0.191140013 \\
\hline $\mathrm{H}$ & -2.809334201 & -3.675182266 & 0.903512065 \\
\hline $\mathrm{H}$ & -4.834063348 & -0.007485001 & 0.0 \\
\hline $\mathrm{H}$ & -4.914938354 & -2.422039173 & 750044 \\
\hline $\mathrm{H}$ & -1.349883097 & 1.876794134 & 1.7 \\
\hline $\mathrm{H}$ & 1.455222104 & 1.513651110 & -1.463619105 \\
\hline $\mathrm{H}$ & -0.928719069 & 4.294232311 & 1.826514130 \\
\hline $\mathrm{H}$ & 1.911748138 & 3.888035278 & -1.337905096 \\
\hline $\mathrm{H}$ & 0.719035050 & 5.314910384 & 0.291123022 \\
\hline C & 1.025464073 & -0.771274057 & -0.096049005 \\
\hline C & 1.970523141 & -0.967254068 & 1.032550074 \\
\hline 0 & 1.667134118 & -0.792661057 & 2.17072615 \\
\hline 0 & 3.162804230 & -1.326426093 & 0.623544047 \\
\hline C & 4.181402303 & -1.461683105 & 1.60 \\
\hline $\mathrm{H}$ & 4.329762309 & -0.523386039 & 2.117126152 \\
\hline $\mathrm{H}$ & 5.069238365 & -1.741167127 & 1.06054807 \\
\hline $\mathrm{H}$ & 3.916652284 & -2.228629159 & 2.316143169 \\
\hline C & 1.404809103 & -1.252320090 & -1.38800410 \\
\hline $\mathrm{N}$ & 1.689531122 & -1.648905121 & -2.41470417 \\
\hline C & 1.059459076 & -0.362829026 & 5.48276739 \\
\hline 0 & 0.201877014 & 0.240595019 & 4.562962330 \\
\hline $\mathrm{H}$ & 0.488100034 & 0.009218003 & 3.68915626 \\
\hline & 1.056618077 & -1.447672105 & 5.39 \\
\hline
\end{tabular}




$\begin{array}{llll}\mathrm{H} & 2.084872148 & -0.014221004 & 5.376898387 \\ \mathrm{H} & 0.713753051 & -0.101915008 & 6.474144465\end{array}$

$\begin{array}{lrrr}\mathrm{V}^{\prime}\left({ }^{1} \pi \pi^{*}\right) / \mathrm{V}\left({ }^{1} \pi \pi^{*}\right) & & \\ \mathrm{C} & -1.607759113 & -0.671990049 & 0.330670025 \\ \mathrm{C} & -1.976007142 & -2.038968148 & 0.665493049 \\ \mathrm{C} & -2.703372193 & 0.175766012 & -0.058402007 \\ \mathrm{C} & -3.326184239 & -2.488927177 & 0.570650039 \\ \mathrm{C} & -4.038826288 & -0.288275022 & -0.138470012 \\ \mathrm{C} & -4.360165315 & -1.633361116 & 0.166021011 \\ \mathrm{C} & -0.012360998 & 1.251021092 & 0.310553024 \\ \mathrm{C} & -0.617940044 & 2.120384153 & 1.227741087 \\ \mathrm{C} & 0.909270064 & 1.781026128 & -0.602388043 \\ \mathrm{C} & -0.324995022 & 3.485356253 & 1.210562089 \\ \mathrm{C} & 1.198627089 & 3.125180225 & -0.612651044 \\ \mathrm{C} & 0.581780039 & 3.991330286 & 0.289582021 \\ \mathrm{C} & -0.302863020 & -0.208242016 & 0.324840021 \\ \mathrm{H} & -1.229368090 & -2.717185196 & 1.017838074 \\ \mathrm{H} & -2.496057179 & 1.180602084 & -0.362405028 \\ \mathrm{H} & -3.540784257 & -3.509322251 & 0.823535057 \\ \mathrm{H} & -4.803052345 & 0.395713026 & -0.452656033 \\ \mathrm{H} & -5.366779386 & -1.989125144 & 0.071520004 \\ \mathrm{H} & -1.271254089 & 1.726202127 & 1.984011144 \\ \mathrm{H} & 1.387299098 & 1.128546082 & -1.311136093 \\ \mathrm{H} & -0.790570056 & 4.138864300 & 1.925774140 \\ \mathrm{H} & 1.907377139 & 3.510017251 & -1.323403094 \\ \mathrm{H} & 0.817464061 & 5.040122360 & 0.277094020 \\ \mathrm{C} & 0.822437057 & -1.120012083 & 0.270673020 \\ \mathrm{C} & 2.074264149 & -0.830737059 & 0.973684069 \\ \mathrm{O} & 2.179127156 & -0.056348005 & 1.883128137 \\ \mathrm{O} & 3.096995223 & -1.519589108 & 0.500052037 \\ \mathrm{C} & 4.357529314 & -1.305269095 & 1.104781081 \\ \mathrm{H} & 4.667417336 & -0.277787019 & 0.978260071 \\ \mathrm{H} & 5.040345361 & -1.968540144 & 0.597686044 \\ \mathrm{H} & 4.319960311 & -1.539723112 & 2.158874157 \\ \mathrm{C} & 0.768825057 & -2.297471166 & -0.525959036 \\ \mathrm{~N} & 0.693752051 & -3.249033234 & -1.150958082 \\ \mathrm{C} & 1.042252074 & -0.242761015 & 5.160216369 \\ \mathrm{O} & 0.308643022 & 0.367134026 & 4.142287298 \\ \mathrm{H} & 0.850868062 & 0.405221029 & 3.362343244 \\ \mathrm{H} & 1.329992097 & -1.260700093 & 4.904436355 \\ \mathrm{H} & 1.943665141 & 0.314504025 & 5.406399389 \\ \mathrm{H} & 0.417502032 & -0.280901021 & 6.042773433 \\ & & & \\ \mathrm{~V}\left({ }^{1} \pi \pi^{*}\right) / T_{2}\left(3 \pi \pi \pi^{*}\right) & & \\ \mathrm{C} & -1.603197113 & -0.672395050 & 0.332472022 \\ \mathrm{C} & -1.972212142 & -2.038350149 & 0.664399049\end{array}$




$\begin{array}{lrrr}\mathrm{C} & -2.703012194 & 0.177922012 & -0.053212006 \\ \mathrm{C} & -3.324298241 & -2.487036178 & 0.571898041 \\ \mathrm{C} & -4.041665292 & -0.286158021 & -0.131722007 \\ \mathrm{C} & -4.360582312 & -1.629680117 & 0.170568015 \\ \mathrm{C} & -0.011343999 & 1.250889089 & 0.307118023 \\ \mathrm{C} & -0.613848043 & 2.118705153 & 1.225637089 \\ \mathrm{C} & 0.906811067 & 1.780759126 & -0.608173046 \\ \mathrm{C} & -0.321741022 & 3.484377248 & 1.207437085 \\ \mathrm{C} & 1.196146083 & 3.125609224 & -0.619126046 \\ \mathrm{C} & 0.581911042 & 3.990722288 & 0.284353019 \\ \mathrm{C} & -0.303546024 & -0.209310018 & 0.324431025 \\ \mathrm{H} & -1.225185087 & -2.717867194 & 1.013297071 \\ \mathrm{H} & -2.495087181 & 1.182688085 & -0.356237028 \\ \mathrm{H} & -3.538735256 & -3.507654253 & 0.823549059 \\ \mathrm{H} & -4.806134347 & 0.398530027 & -0.443216030 \\ \mathrm{H} & -5.367050388 & -1.986237144 & 0.077530007 \\ \mathrm{H} & -1.264638093 & 1.724158126 & 1.984000142 \\ \mathrm{H} & 1.383630101 & 1.128715080 & -1.318155095 \\ \mathrm{H} & -0.786084056 & 4.137581299 & 1.923693140 \\ \mathrm{H} & 1.902950138 & 3.510662255 & -1.331666096 \\ \mathrm{H} & 0.817138061 & 5.039649361 & 0.271447021 \\ \mathrm{C} & 0.824532059 & -1.121655083 & 0.270951018 \\ \mathrm{C} & 2.074587148 & -0.833130062 & 0.976358071 \\ \mathrm{O} & 2.177864159 & -0.059252003 & 1.886429134 \\ \mathrm{O} & 3.097904223 & -1.521904111 & 0.503701037 \\ \mathrm{C} & 4.357717314 & -1.306958096 & 1.109655078 \\ \mathrm{H} & 4.666246336 & -0.278856021 & 0.984993068 \\ \mathrm{H} & 5.041559362 & -1.968681143 & 0.601916043 \\ \mathrm{H} & 4.319437310 & -1.543068110 & 2.163358156 \\ \mathrm{C} & 0.772707053 & -2.296117165 & -0.529088040 \\ \mathrm{~N} & 0.698458051 & -3.246720232 & -1.156015085 \\ \mathrm{C} & 1.040448077 & -0.246208018 & 5.159919369 \\ \mathrm{O} & 0.305262024 & 0.363596026 & 4.143071296 \\ \mathrm{H} & 0.847189063 & 0.403424028 & 3.362891244 \\ \mathrm{H} & 1.329375097 & -1.263494091 & 4.902794355 \\ \mathrm{H} & 1.941160138 & 0.312271024 & 5.405929390 \\ \mathrm{H} & 0.416142031 & -0.285983023 & 6.042743434 \\ & & & \\ \mathrm{~V}{ }^{\prime}\left(1 \pi \pi^{*}\right) / \mathrm{T}_{2}\left({ }^{3} \pi \pi^{*}\right) & & \\ \mathrm{C} & -1.642566117 & -0.658801047 & 0.252363016 \\ \mathrm{C} & -2.093472151 & -1.955012141 & 0.594389042 \\ \mathrm{C} & -2.667149190 & 0.233736019 & -0.198062017 \\ \mathrm{C} & -3.460605252 & -2.423945174 & 0.396059029 \\ \mathrm{C} & -4.036828289 & -0.198727012 & -0.445067034 \\ \mathrm{C} & -4.449777323 & -1.545476110 & -0.170421014 \\ \mathrm{C} & 0.011548002 & 1.214941089 & 0.376948025 \\ \mathrm{C} & -0.425179029 & 2.068644149 & 1.395766098\end{array}$




$\begin{array}{lrrr}\mathrm{C} & 0.760502056 & 1.748394127 & -0.666815050 \\ \mathrm{C} & -0.118847008 & 3.426050247 & 1.358163096 \\ \mathrm{C} & 1.079492076 & 3.110302221 & -0.688322052 \\ \mathrm{C} & 0.638320046 & 3.942963284 & 0.311847021 \\ \mathrm{C} & -0.328609023 & -0.252735017 & 0.407157029 \\ \mathrm{H} & -1.428217105 & -2.635256191 & 1.083984076 \\ \mathrm{H} & -2.423041175 & 1.255134091 & -0.404364027 \\ \mathrm{H} & -3.711658268 & -3.418033243 & 0.706058052 \\ \mathrm{H} & -4.731336339 & 0.504831037 & -0.859517062 \\ \mathrm{H} & -5.445507392 & -1.880557134 & -0.380016026 \\ \mathrm{H} & -0.982418071 & 1.667920121 & 2.222726160 \\ \mathrm{H} & 1.100377077 & 1.112649079 & -1.465892106 \\ \mathrm{H} & -0.460298035 & 4.075056292 & 2.144100152 \\ \mathrm{H} & 1.672070120 & 3.503146250 & -1.494503106 \\ \mathrm{H} & 0.881465064 & 4.990737359 & 0.289158023 \\ \mathrm{C} & 0.781340056 & -1.179360084 & 0.500833034 \\ \mathrm{C} & 2.114904152 & -0.780446059 & 1.044271075 \\ \mathrm{O} & 2.270483163 & 0.004710000 & 1.924887138 \\ \mathrm{O} & 3.093957222 & -1.421618100 & 0.450160031 \\ \mathrm{C} & 4.415108318 & -1.174248083 & 0.907640065 \\ \mathrm{H} & 4.672554335 & -0.135784009 & 0.760256052 \\ \mathrm{H} & 5.050490361 & -1.810266129 & 0.312774023 \\ \mathrm{H} & 4.501986326 & -1.424211101 & 1.954858139 \\ \mathrm{C} & 0.724155051 & -2.513472180 & -0.034532001 \\ \mathrm{~N} & 0.702170049 & -3.567774254 & -0.458700031 \\ \mathrm{C} & 1.105952081 & -0.304934024 & 5.249305375 \\ \mathrm{O} & 0.395946028 & 0.356590026 & 4.247089307 \\ \mathrm{H} & 0.957038068 & 0.456853033 & 3.487426251 \\ \mathrm{H} & 1.392813099 & -1.311888096 & 4.952254357 \\ \mathrm{H} & 2.003127143 & 0.236234016 & 5.541895398 \\ \mathrm{H} & 0.460765034 & -0.381210030 & 6.113900438 \\ & & & \\ \mathrm{~T}_{2}\left({ }^{3} \mathrm{~T} \pi^{*}\right) / \mathrm{T}_{1}\left({ }^{3} \mathrm{~m} \pi \pi^{*}\right) & & \\ \mathrm{C} & -1.708031122 & -0.629241047 & 0.206835013 \\ \mathrm{C} & -2.238540162 & -1.777398126 & 0.819850057 \\ \mathrm{C} & -2.565946184 & 0.164201012 & -0.575851042 \\ \mathrm{C} & -3.554183258 & -2.127292155 & 0.645348049 \\ \mathrm{C} & -3.894279279 & -0.205253017 & -0.765988053 \\ \mathrm{C} & -4.395187318 & -1.352188098 & -0.155895009 \\ \mathrm{C} & 0.003921002 & 1.237125088 & 0.460846035 \\ \mathrm{C} & -0.552764039 & 2.054699149 & 1.406560102 \\ \mathrm{C} & 0.902225063 & 1.805609131 & -0.546826041 \\ \mathrm{C} & -0.257279020 & 3.479484248 & 1.422469102 \\ \mathrm{C} & 1.238294088 & 3.236939232 & -0.477438034 \\ \mathrm{C} & 0.691080050 & 4.022721292 & 0.437407029 \\ \mathrm{C} & -0.300763022 & -0.233388016 & 0.412924030 \\ \mathrm{H} & -1.620805116 & -2.375219173 & 1.461532104 \\ & & & \end{array}$




$\begin{array}{rrrr}\mathrm{H} & -2.193109157 & 1.056078078 & -1.042718078 \\ \mathrm{H} & -3.937808283 & -3.001162218 & 1.139544084 \\ \mathrm{H} & -4.531352324 & 0.399944031 & -1.384069101 \\ \mathrm{H} & -5.421516390 & -1.638170120 & -0.295644021 \\ \mathrm{H} & -1.200595088 & 1.649734120 & 2.160680154 \\ \mathrm{H} & 1.272147091 & 1.199667086 & -1.350364098 \\ \mathrm{H} & -0.717676054 & 4.120919296 & 2.145590157 \\ \mathrm{H} & 1.936374137 & 3.628982261 & -1.194454087 \\ \mathrm{H} & 0.933213069 & 5.069225363 & 0.479691032 \\ \mathrm{C} & 0.728590054 & -1.147286084 & 0.473917036 \\ \mathrm{C} & 2.112570153 & -0.774322055 & 0.912902066 \\ \mathrm{O} & 2.342319170 & -0.060913006 & 1.837461130 \\ \mathrm{O} & 3.038002221 & -1.354171100 & 0.187073016 \\ \mathrm{C} & 4.389703317 & -1.152041081 & 0.573535040 \\ \mathrm{H} & 4.640256335 & -0.102732005 & 0.524761037 \\ \mathrm{H} & 4.979283360 & -1.716173122 & -0.130582011 \\ \mathrm{H} & 4.549326327 & -1.516387110 & 1.577594116 \\ \mathrm{C} & 0.578490044 & -2.529145182 & 0.089841004 \\ \mathrm{~N} & 0.511414039 & -3.620645258 & -0.215361017 \\ \mathrm{C} & 1.153798081 & -0.277420019 & 5.211047374 \\ \mathrm{O} & 0.473879036 & 0.371271028 & 4.178956303 \\ \mathrm{H} & 1.040404075 & 0.413945029 & 3.418157246 \\ \mathrm{H} & 1.410062102 & -1.302621092 & 4.951234357 \\ \mathrm{H} & 2.066652146 & 0.243953019 & 5.490916394 \\ \mathrm{H} & 0.500249034 & -0.301340021 & 6.072415434\end{array}$

\begin{tabular}{lrrr}
\multicolumn{4}{l}{$\mathrm{T}_{1}\left({ }^{3} \mathrm{Tm} \mathrm{T}^{*} / \mathrm{S}_{0}\right.$} \\
$\mathrm{C}$ & -1.445999106 & -0.600720045 & 0.242626017 \\
$\mathrm{C}$ & -1.501793110 & -1.994734143 & 0.493684034 \\
$\mathrm{C}$ & -2.676188194 & 0.081069007 & 0.085920007 \\
$\mathrm{C}$ & -2.697264196 & -2.650615190 & 0.605696041 \\
$\mathrm{C}$ & -3.886974278 & -0.594631045 & 0.199457012 \\
$\mathrm{C}$ & -3.906339281 & -1.961546141 & 0.462933031 \\
$\mathrm{C}$ & -0.008956002 & 1.549124110 & 0.171521010 \\
$\mathrm{C}$ & -0.687307048 & 2.326697165 & 1.123846083 \\
$\mathrm{C}$ & 0.906921067 & 2.193845158 & -0.677176050 \\
$\mathrm{C}$ & -0.469692032 & 3.702176264 & 1.200254086 \\
$\mathrm{C}$ & 1.126198080 & 3.547521255 & -0.590438041 \\
$\mathrm{C}$ & 0.437092031 & 4.317832310 & 0.344966024 \\
$\mathrm{C}$ & -0.184889011 & 0.083929004 & 0.103899005 \\
$\mathrm{H}$ & -0.590837040 & -2.551537185 & 0.617514045 \\
$\mathrm{H}$ & -2.678021195 & 1.126320082 & -0.150676009 \\
$\mathrm{H}$ & -2.707643193 & -3.705931269 & 0.810596060 \\
$\mathrm{H}$ & -4.808294348 & -0.055280004 & 0.073826005 \\
$\mathrm{H}$ & -4.839264346 & -2.485749177 & 0.549988039 \\
$\mathrm{H}$ & -1.356557096 & 1.856934133 & 1.819346133 \\
$\mathrm{H}$ & 1.433864103 & 1.628380119 & -1.424069101
\end{tabular}




$\begin{array}{lrrr}\mathrm{H} & -0.995242073 & 4.282892308 & 1.935780141 \\ \mathrm{H} & 1.831163132 & 4.011714289 & -1.255554088 \\ \mathrm{H} & 0.612899043 & 5.375881388 & 0.408752028 \\ \mathrm{C} & 1.067088076 & -0.697646049 & -0.092901008 \\ \mathrm{C} & 1.990935142 & -0.930219069 & 1.046003078 \\ \mathrm{O} & 1.668016119 & -0.778814055 & 2.182403155 \\ \mathrm{O} & 3.185043227 & -1.301310092 & 0.652950045 \\ \mathrm{C} & 4.176558299 & -1.487573107 & 1.652826118 \\ \mathrm{H} & 4.332961313 & -0.567709042 & 2.196725158 \\ \mathrm{H} & 5.070953365 & -1.771163126 & 1.122908079 \\ \mathrm{H} & 3.877654281 & -2.268190164 & 2.336745167 \\ \mathrm{C} & 1.460566107 & -1.140656081 & -1.394136100 \\ \mathrm{~N} & 1.756868128 & -1.497459106 & -2.432324177 \\ \mathrm{C} & 1.043141075 & -0.368120026 & 5.485412395 \\ \mathrm{O} & 0.189891012 & 0.243802018 & 4.567075328 \\ \mathrm{H} & 0.474082034 & 0.010826003 & 3.692912265 \\ \mathrm{H} & 1.015883071 & -1.453535103 & 5.411053392 \\ \mathrm{H} & 2.074649147 & -0.043085003 & 5.365523389 \\ \mathrm{H} & 0.712603054 & -0.088819004 & 6.477030466\end{array}$

$S_{1}\left({ }^{1} \pi \pi^{*}\right)$ state minimum-energy photoisomerization path 20 degree
$-1.613121118$
$-0.661589049$
0.265029022
C -2.097939153
$-1.974228143$
0.632380047
C $\quad-2.644590189$
0.232343018
$-0.215958013$
C $\quad-3.453628251$
$-2.401826173$
0.387030027
C $\quad-3.996075286$
$-0.206850015$
$-0.461565034$
C $\quad-4.409935319$
$-1.529045109$
$-0.182198013$
C $\quad 0.016878003$
1.216048085
0.407126030
C $\quad-0.458655035$
2.064594149
1.414997103
C $\quad 0.773647057$
1.760243125
$-0.627581045$
C $\quad-0.177294011$
3.426397244
1.373697100
C $\quad 1.059589074$
3.124725222
$-0.653240045$
C $\quad 0.584220044$
3.954231283
0.336740027
C $\quad-0.286136018$
$-0.261437019$
0.418874030
$\mathrm{H} \quad-1.456080107$
$-2.649527191$
1.154400084
$\mathrm{H} \quad-2.399445172$
1.252013088
$-0.426941030$
H $\quad-3.721638265$
$-3.401556246$
0.667573049
H $\quad-4.688245335$
0.500371036
$-0.875210062$
$\mathrm{H} \quad-5.409816387$
$-1.855431132$
$-0.386854028$
H $\quad-1.012906073$
1.657608118
2.240619162
H $\quad 1.147729085$
1.123448083
$-1.409445103$
H $\quad-0.541098041$
4.070536293
2.153501156
H $\quad 1.657151121$
3.525131252
$-1.452175102$
H 0.805510058
5.006531358
0.312109022
C $\quad 0.782936055$
$-1.163341084$
0.501695037
C 2.115563153
$-0.766934058$
1.001672074 


$\begin{array}{lrrr}\mathrm{O} & 2.311164167 & 0.065550006 & 1.838041135 \\ \mathrm{O} & 3.090891222 & -1.459771103 & 0.444969030 \\ \mathrm{C} & 4.407376318 & -1.201515084 & 0.894961063 \\ \mathrm{H} & 4.675011337 & -0.171398013 & 0.709528052 \\ \mathrm{H} & 5.043534363 & -1.863280132 & 0.328665025 \\ \mathrm{H} & 4.495677321 & -1.409344104 & 1.951631142 \\ \mathrm{C} & 0.686021052 & -2.505400179 & 0.013677999 \\ \mathrm{~N} & 0.600021044 & -3.572177258 & -0.377052025 \\ \mathrm{C} & 1.119189080 & -0.298249023 & 5.184062372 \\ \mathrm{O} & 0.437939032 & 0.358254024 & 4.159080299 \\ \mathrm{H} & 1.011373073 & 0.427249032 & 3.403645247 \\ \mathrm{H} & 1.391593102 & -1.315354096 & 4.908877354 \\ \mathrm{H} & 2.023154147 & 0.230068017 & 5.479288396 \\ \mathrm{H} & 0.460939033 & -0.346248024 & 6.041425434\end{array}$

30 degree

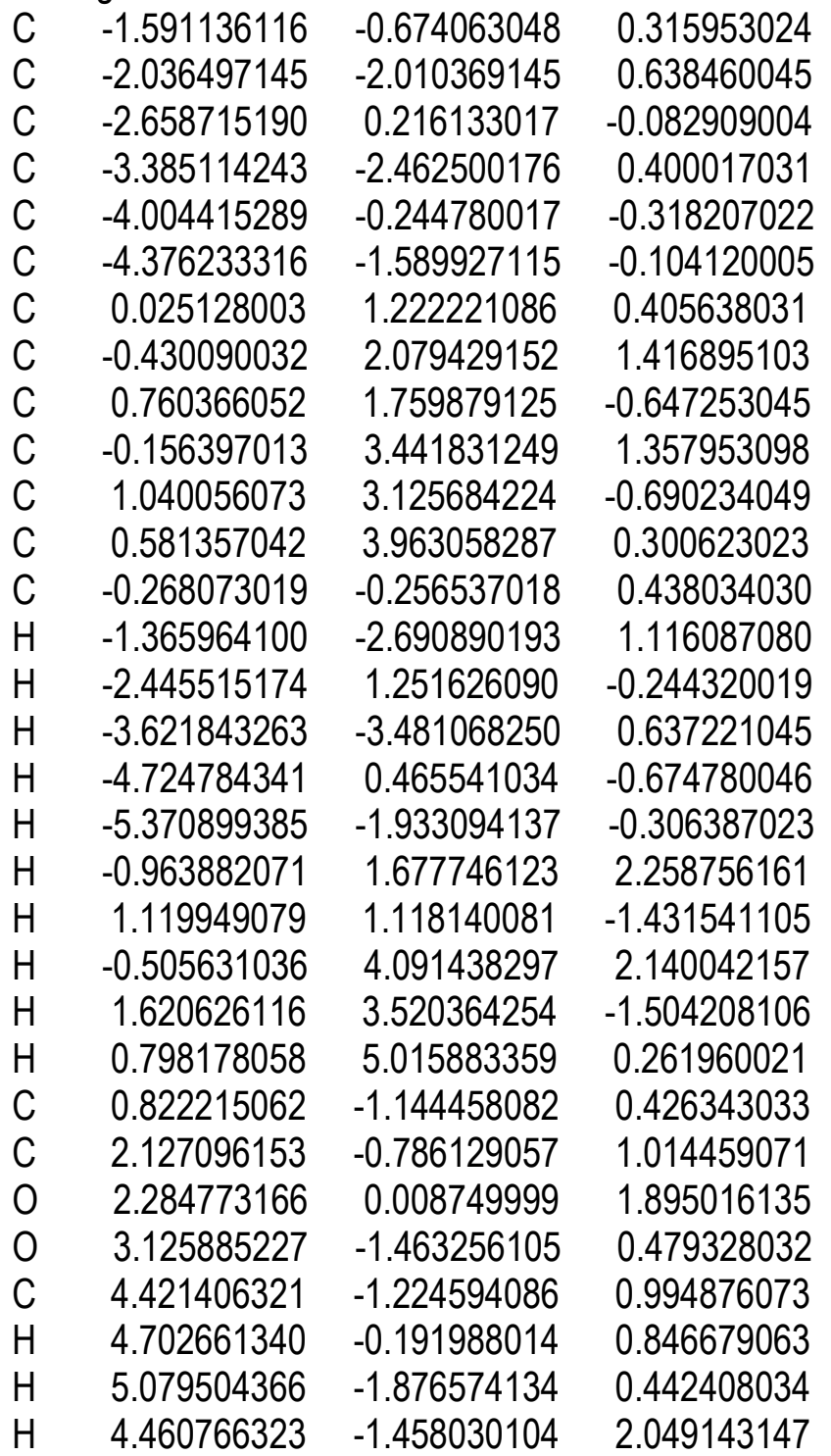




$\begin{array}{rrrr}\mathrm{C} & 0.731492051 & -2.460753177 & -0.125484007 \\ \mathrm{~N} & 0.646902047 & -3.508550250 & -0.565609039 \\ \mathrm{C} & 1.103242080 & -0.312801022 & 5.211334373 \\ \mathrm{O} & 0.411686030 & 0.326505024 & 4.182590300 \\ \mathrm{H} & 0.978953071 & 0.381482027 & 3.421238248 \\ \mathrm{H} & 1.373909099 & -1.334037096 & 4.949917357 \\ \mathrm{H} & 2.009820145 & 0.220602015 & 5.488877394 \\ \mathrm{H} & 0.453829034 & -0.347538026 & 6.076022439\end{array}$

40 degree

$\begin{array}{lrrr}\text { C } & -1.557475114 & -0.685165049 & 0.368705026 \\ \mathrm{C} & -1.958983140 & -2.043496149 & 0.644602047 \\ \mathrm{C} & -2.661538191 & 0.192895013 & 0.055885002 \\ \mathrm{C} & -3.299156236 & -2.523660181 & 0.417991029 \\ \mathrm{C} & -4.001461289 & -0.292964019 & -0.159083011 \\ \mathrm{C} & -4.328421311 & -1.656686117 & -0.010843001 \\ \mathrm{C} & 0.035942005 & 1.236114090 & 0.405716027 \\ \mathrm{C} & -0.401023029 & 2.101692152 & 1.419214101 \\ \mathrm{C} & 0.750394056 & 1.768530126 & -0.663983047 \\ \mathrm{C} & -0.136784011 & 3.464945251 & 1.342906096 \\ \mathrm{C} & 1.021890074 & 3.135618225 & -0.724140054 \\ \mathrm{C} & 0.577673042 & 3.980262284 & 0.266833019 \\ \mathrm{C} & -0.240536016 & -0.244914015 & 0.457507033 \\ \mathrm{H} & -1.255228088 & -2.727069195 & 1.067007075 \\ \mathrm{H} & -2.482494181 & 1.241197091 & -0.055047002 \\ \mathrm{H} & -3.499642250 & -3.560140259 & 0.606006044 \\ \mathrm{H} & -4.752318344 & 0.415439029 & -0.451221032 \\ \mathrm{H} & -5.318523384 & -2.017947143 & -0.203112013 \\ \mathrm{H} & -0.914058065 & 1.705119123 & 2.276570162 \\ \mathrm{H} & 1.098716080 & 1.122057083 & -1.449178107 \\ \mathrm{H} & -0.472728033 & 4.119548298 & 2.126783155 \\ \mathrm{H} & 1.585964112 & 3.524980251 & -1.552074114 \\ \mathrm{H} & 0.788798058 & 5.033650364 & 0.214525017 \\ \mathrm{C} & 0.868168062 & -1.113267078 & 0.354109026 \\ \mathrm{C} & 2.143122153 & -0.812019060 & 1.028930073 \\ \mathrm{O} & 2.263736164 & -0.065249005 & 1.956859138 \\ \mathrm{O} & 3.161468227 & -1.473250104 & 0.510442035 \\ \mathrm{C} & 4.433898317 & -1.263075088 & 1.091063076 \\ \mathrm{H} & 4.722882341 & -0.225804017 & 1.001915072 \\ \mathrm{H} & 5.114135366 & -1.891555136 & 0.538133039 \\ \mathrm{H} & 4.428309316 & -1.544169111 & 2.134348151 \\ \mathrm{C} & 0.780132056 & -2.396385173 & -0.269930018 \\ \mathrm{~N} & 0.693038048 & -3.418098248 & -0.768037053 \\ \mathrm{C} & 1.090119077 & -0.316790023 & 5.249772380 \\ \mathrm{O} & 0.378360029 & 0.277364022 & 4.207830303 \\ \mathrm{H} & 0.939287066 & 0.315575022 & 3.440720247 \\ \mathrm{H} & 1.376620100 & -1.340803098 & 5.017877363\end{array}$




$\begin{array}{rrrr}\mathrm{H} & 1.989657142 & 0.240672020 & 5.501601398 \\ \mathrm{H} & 0.449373034 & -0.335701024 & 6.121387439\end{array}$

50 degree

$\begin{array}{lrrr}\mathrm{C} & -1.504256108 & -0.690485048 & 0.419050029 \\ \mathrm{C} & -1.859244134 & -2.067697150 & 0.648510047 \\ \mathrm{C} & -2.642958191 & 0.168182013 & 0.191179013 \\ \mathrm{C} & -3.191290227 & -2.576518188 & 0.444831031 \\ \mathrm{C} & -3.976199288 & -0.345758027 & 0.004582003 \\ \mathrm{C} & -4.258755306 & -1.721816126 & 0.095645009 \\ \mathrm{C} & 0.055107005 & 1.260928089 & 0.405561031 \\ \mathrm{C} & -0.366395025 & 2.131282151 & 1.422895100 \\ \mathrm{C} & 0.744494053 & 1.793227129 & -0.681079049 \\ \mathrm{C} & -0.118332008 & 3.496436251 & 1.331589096 \\ \mathrm{C} & 1.001189073 & 3.162519226 & -0.755846053 \\ \mathrm{C} & 0.568935040 & 4.010622289 & 0.237214016 \\ \mathrm{C} & -0.195941016 & -0.222824014 & 0.475549035 \\ \mathrm{H} & -1.120913081 & -2.750066196 & 1.009809074 \\ \mathrm{H} & -2.497138182 & 1.225165090 & 0.124500008 \\ \mathrm{H} & -3.355760242 & -3.626455259 & 0.587934043 \\ \mathrm{H} & -4.756852345 & 0.354376028 & -0.223039019 \\ \mathrm{H} & -5.244819376 & -2.103084153 & -0.077236007 \\ \mathrm{H} & -0.854961063 & 1.735683125 & 2.295123164 \\ \mathrm{H} & 1.083342078 & 1.145163083 & -1.468820108 \\ \mathrm{H} & -0.443097034 & 4.153325301 & 2.118315155 \\ \mathrm{H} & 1.546025110 & 3.550432253 & -1.597127117 \\ \mathrm{H} & 0.769881056 & 5.065305366 & 0.173099011 \\ \mathrm{C} & 0.929186068 & -1.066757076 & 0.287446023 \\ \mathrm{C} & 2.170978155 & -0.843803059 & 1.045769075 \\ \mathrm{O} & 2.256108164 & -0.156802014 & 2.023214145 \\ \mathrm{O} & 3.204009230 & -1.491972110 & 0.539378040 \\ \mathrm{C} & 4.451246319 & -1.326528097 & 1.184773085 \\ \mathrm{H} & 4.751943342 & -0.288917019 & 1.164523086 \\ \mathrm{H} & 5.150152371 & -1.930977137 & 0.628258048 \\ \mathrm{H} & 4.398713316 & -1.663407118 & 2.210110162 \\ \mathrm{C} & 0.840735059 & -2.307517167 & -0.415885030 \\ \mathrm{~N} & 0.748805054 & -3.295710239 & -0.977484070 \\ \mathrm{C} & 1.076261078 & -0.308095022 & 5.294522381 \\ \mathrm{O} & 0.345222024 & 0.217271013 & 4.229076304 \\ \mathrm{H} & 0.903672067 & 0.231990015 & 3.459261247 \\ \mathrm{H} & 1.390290098 & -1.333385097 & 5.108673368 \\ \mathrm{H} & 1.960941143 & 0.284761020 & 5.516178395 \\ \mathrm{H} & 0.439173031 & -0.304479022 & 6.169064441\end{array}$

60 degree

$\begin{array}{llll}\text { C } & -1.514273110 & -0.800936056 & 0.260851019 \\ \text { C } & -1.636929120 & -2.196578157 & 0.528646040\end{array}$

S15 


$\begin{array}{lrrr}\text { C } & -2.713745194 & -0.025689000 & 0.242016019 \\ \text { C } & -2.845734203 & -2.750017199 & 0.875300065 \\ \text { C } & -3.931243283 & -0.605319045 & 0.543509041 \\ \text { C } & -3.997037288 & -1.966632143 & 0.881220066 \\ \text { C } & 0.010950000 & 1.206180088 & 0.112993006 \\ \text { C } & -0.552929042 & 1.904511136 & 1.181980083 \\ \text { C } & 0.857437062 & 1.855954134 & -0.744702051 \\ \text { C } & -0.261573018 & 3.264183235 & 1.366385098 \\ \text { C } & 1.130408082 & 3.233540231 & -0.578335043 \\ \text { C } & 0.566631039 & 3.926720284 & 0.475735035 \\ \text { C } & -0.229015017 & -0.257018019 & -0.018153002 \\ \text { H } & -0.758463057 & -2.804104199 & 0.461038032 \\ \text { H } & -2.676425191 & 1.008381073 & -0.033253001 \\ \text { H } & -2.906188211 & -3.794755273 & 1.114668080 \\ \text { H } & -4.830562349 & -0.018738002 & 0.518977035 \\ \text { H } & -4.944387354 & -2.413361174 & 1.115737082 \\ \text { H } & -1.168882083 & 1.399673099 & 1.899543136 \\ \text { H } & 1.314759095 & 1.327034097 & -1.558769111 \\ \text { H } & -0.685463050 & 3.783910272 & 2.205526158 \\ \text { H } & 1.786391128 & 3.724636266 & -1.271581094 \\ \text { H } & 0.777768057 & 4.972509357 & 0.609790042 \\ \text { C } & 0.943216070 & -1.160311084 & -0.044451005 \\ \text { C } & 1.812592133 & -1.164746081 & 1.083805077 \\ \text { O } & 1.508295111 & -0.798820056 & 2.202217160 \\ \text { O } & 3.024015219 & -1.647460118 & 0.819577060 \\ \text { C } & 3.932829280 & -1.739135123 & 1.890145138 \\ \text { H } & 4.110858297 & -0.771802054 & 2.336665166 \\ \text { H } & 4.846279347 & -2.125590151 & 1.463354104 \\ \text { H } & 3.564803258 & -2.416444176 & 2.649565191 \\ \text { C } & 1.571724111 & -1.216873088 & -1.316844095 \\ \text { N } & 2.023023144 & -1.265089089 & -2.366697170 \\ \text { C } & 0.269901019 & -1.259848091 & 5.343946382 \\ \text { O } & -0.435329029 & -0.597042040 & 4.338605311 \\ \text { H } & 0.115011008 & -0.583978041 & 3.561903255 \\ \text { H } & 0.519589039 & -2.281081165 & 5.061765366 \\ \text { H } & 1.191720087 & -0.744982055 & 5.604855404 \\ \text { H } & -0.356924027 & -1.298662094 & 6.225946450\end{array}$

70 degree

$\begin{array}{lrrr}C & -1.500066109 & -0.798481060 & 0.307189023 \\ C & -1.609890115 & -2.185263157 & 0.566578042 \\ C & -2.706450195 & -0.027556000 & 0.276670022 \\ C & -2.828374205 & -2.758781200 & 0.856469059 \\ C & -3.925905282 & -0.620792045 & 0.539971041 \\ C & -3.987220288 & -1.985995140 & 0.846601063 \\ C & 0.000081001 & 1.231014091 & 0.133376009 \\ C & -0.556857040 & 1.962216143 & 1.202989086\end{array}$

S16 


$\begin{array}{rrrr}\text { C } & 0.839385059 & 1.870907132 & -0.748460056 \\ \text { C } & -0.269891018 & 3.326656239 & 1.358429098 \\ \text { C } & 1.103587077 & 3.240827235 & -0.606043044 \\ \text { C } & 0.548649040 & 3.962547287 & 0.447250033 \\ \text { C } & -0.217456013 & -0.235326017 & 0.053114002 \\ \text { H } & -0.722881052 & -2.784634202 & 0.546043038 \\ \text { H } & -2.670562193 & 1.010246074 & 0.015474000 \\ \text { H } & -2.887168210 & -3.809692275 & 1.073253076 \\ \text { H } & -4.828447349 & -0.038799001 & 0.503386034 \\ \text { H } & -4.935799357 & -2.445958175 & 1.051766077 \\ \text { H } & -1.157991081 & 1.466998108 & 1.939850138 \\ \text { H } & 1.289532093 & 1.332124094 & -1.559806114 \\ \text { H } & -0.688000048 & 3.870144277 & 2.185843157 \\ \text { H } & 1.751810125 & 3.725389269 & -1.311048096 \\ \text { H } & 0.764104055 & 5.009792360 & 0.557361039 \\ \mathrm{C} & 0.976952072 & -1.083938080 & -0.056633004 \\ \mathrm{C} & 1.813981133 & -1.177441086 & 1.094253078 \\ \text { O } & 1.491600109 & -0.862225063 & 2.222178160 \\ \text { O } & 3.014814219 & -1.683704119 & 0.827648061 \\ \mathrm{C} & 3.911259280 & -1.826444130 & 1.902929135 \\ \mathrm{H} & 4.108384293 & -0.873041065 & 2.370877173 \\ \mathrm{H} & 4.818822345 & -2.224769160 & 1.475164107 \\ \mathrm{H} & 3.523792254 & -2.511156183 & 2.645348188 \\ \mathrm{C} & 1.550068109 & -1.221421086 & -1.336910095 \\ \mathrm{~N} & 1.984845143 & -1.316572093 & -2.389836171 \\ \mathrm{C} & 0.229561017 & -1.331601097 & 5.333834382 \\ \mathrm{O} & -0.467732034 & -0.654011046 & 4.333153314 \\ \mathrm{H} & 0.086091005 & -0.635694046 & 3.559547257 \\ \mathrm{H} & 0.464105032 & -2.354786168 & 5.046884362 \\ \mathrm{H} & 1.159854081 & -0.831757059 & 5.594289402 \\ \mathrm{H} & -0.395394028 & -1.364046097 & 6.216881448\end{array}$

80 degree

$\begin{array}{lrrr}\text { C } & -1.495775106 & -0.760564055 & 0.332163025 \\ \text { C } & -1.617925116 & -2.150673153 & 0.527965036 \\ \text { C } & -2.691331193 & 0.012654999 & 0.310064022 \\ \text { C } & -2.841356203 & -2.729451196 & 0.766653054 \\ \text { C } & -3.922954283 & -0.581935041 & 0.543442037 \\ \text { C } & -3.997113288 & -1.954686141 & 0.783883054 \\ \text { C } & -0.000894997 & 1.271004090 & 0.163168011 \\ \text { C } & -0.517135038 & 2.030967147 & 1.186197085 \\ \text { C } & 0.848380061 & 1.875648137 & -0.765098056 \\ \text { C } & -0.198033012 & 3.386045246 & 1.291614094 \\ \text { C } & 1.120693081 & 3.249575233 & -0.698295048 \\ \text { C } & 0.615760045 & 4.009222287 & 0.322828026 \\ \text { C } & -0.214941014 & -0.175708014 & 0.100889008 \\ \text { H } & -0.732048053 & -2.753949198 & 0.498925038\end{array}$




$\begin{array}{lrrr}\mathrm{H} & -2.657090192 & 1.061353076 & 0.081798007 \\ \mathrm{H} & -2.904411207 & -3.788286272 & 0.937409069 \\ \mathrm{H} & -4.820071346 & 0.012590000 & 0.533655041 \\ \mathrm{H} & -4.948295354 & -2.418328174 & 0.965331070 \\ \mathrm{H} & -1.113815078 & 1.571424115 & 1.954188143 \\ \mathrm{H} & 1.267130089 & 1.279482091 & -1.552459112 \\ \mathrm{H} & -0.570559042 & 3.958346287 & 2.122022153 \\ \mathrm{H} & 1.740899125 & 3.688768267 & -1.455142103 \\ \mathrm{H} & 0.844346058 & 5.054591363 & 0.390472028 \\ \mathrm{C} & 1.007219074 & -1.001183072 & -0.044989004 \\ \mathrm{C} & 1.762000126 & -1.246277092 & 1.127683084 \\ \mathrm{O} & 1.368845100 & -1.008390074 & 2.241195160 \\ \mathrm{O} & 2.962741214 & -1.756239127 & 0.897453065 \\ \mathrm{C} & 3.800857274 & -1.970607142 & 2.011006142 \\ \mathrm{H} & 3.995114289 & -1.053448078 & 2.548115181 \\ \mathrm{H} & 4.720591342 & -2.364738172 & 1.609146113 \\ \mathrm{H} & 3.356805240 & -2.688789195 & 2.687431194 \\ \mathrm{C} & 1.559302115 & -1.232126088 & -1.313234096 \\ \mathrm{~N} & 1.978599142 & -1.436331101 & -2.371712173 \\ \mathrm{C} & 0.016061000 & -1.652107120 & 5.303168380 \\ \mathrm{O} & -0.647574049 & -0.886990066 & 4.356195316 \\ \mathrm{H} & -0.081348005 & -0.840077059 & 3.576109256 \\ \mathrm{H} & 0.219305017 & -2.657767190 & 4.948639356 \\ \mathrm{H} & 0.969716071 & -1.206268085 & 5.612924405 \\ \mathrm{H} & -0.612145045 & -1.723879123 & 6.177263445\end{array}$

90 degree

$\begin{array}{lrrr}\text { C } & -1.375025097 & -0.704067049 & 0.394895029 \\ \text { C } & -1.420043105 & -2.106547152 & 0.548281040 \\ \text { C } & -2.614741190 & 0.001039002 & 0.339348024 \\ \text { C } & -2.615626186 & -2.770471201 & 0.687250050 \\ \text { C } & -3.815081273 & -0.680259047 & 0.476958033 \\ \text { C } & -3.815731277 & -2.066245151 & 0.658963049 \\ \text { C } & -0.005634002 & 1.418153101 & 0.250587018 \\ \text { C } & -0.614664045 & 2.172527157 & 1.242188092 \\ \text { C } & 0.821917061 & 2.050930147 & -0.664287049 \\ \text { C } & -0.407616027 & 3.555192256 & 1.313872093 \\ \text { C } & 0.997127071 & 3.453379246 & -0.623129045 \\ \text { C } & 0.387892030 & 4.192769300 & 0.364434026 \\ \text { C } & -0.121789006 & -0.050937004 & 0.235998019 \\ \text { H } & -0.497004034 & -2.647503188 & 0.556604040 \\ \text { H } & -2.628372187 & 1.054756077 & 0.155316010 \\ \text { H } & -2.625395190 & -3.835762277 & 0.818100058 \\ \text { H } & -4.744680342 & -0.144167012 & 0.431832030 \\ \text { H } & -4.744763344 & -2.594054185 & 0.760316056 \\ \text { H } & -1.204405085 & 1.686912123 & 1.997469143 \\ \text { H } & 1.321107094 & 1.479505107 & -1.419799101\end{array}$

S18 


$\begin{array}{rrrr}\mathrm{H} & -0.855802063 & 4.119539297 & 2.110250150 \\ \mathrm{H} & 1.624327119 & 3.924226282 & -1.355513098 \\ \mathrm{H} & 0.538739037 & 5.255723380 & 0.412483029 \\ \mathrm{C} & 1.138097080 & -0.792768057 & 0.089778005 \\ \mathrm{C} & 1.826779131 & -1.143952083 & 1.270738094 \\ \mathrm{O} & 1.392326102 & -0.966438071 & 2.394934174 \\ \mathrm{O} & 3.026546216 & -1.684757123 & 1.068406077 \\ \mathrm{C} & 3.801394273 & -1.985527141 & 2.202873160 \\ \mathrm{H} & 3.980085285 & -1.100230077 & 2.796758203 \\ \mathrm{H} & 4.735745343 & -2.371471169 & 1.823958130 \\ \mathrm{H} & 3.316533237 & -2.732150196 & 2.816523201 \\ \mathrm{C} & 1.667219120 & -1.031729072 & -1.189485086 \\ \mathrm{~N} & 2.052873149 & -1.211136087 & -2.252092163 \\ \mathrm{C} & -0.535680038 & -2.231506163 & 5.041701362 \\ \mathrm{O} & -0.979407068 & -1.420070103 & 3.998502289 \\ \mathrm{H} & -0.225935015 & -1.166359082 & 3.474242250 \\ \mathrm{H} & 0.033312004 & -3.087058222 & 4.683680340 \\ \mathrm{H} & 0.080727005 & -1.686335119 & 5.753753415 \\ \mathrm{H} & -1.404842102 & -2.604357187 & 5.569164399\end{array}$

LIIC path connecting the Franck-Condon point and $\mathrm{V}\left({ }^{1} \mathrm{~T} \pi^{*}\right) / \mathrm{T}_{2}\left({ }^{3} \mathrm{~m} \mathrm{~m}^{*}\right)$ crossing point Coord_1
C -1.681776120
$-0.640966049$
0.179419012
0.832706059
C -2.501392180
$-1.743826126$
0.133228008
$-0.645971044$
C -3.558730255
$-2.087840149$
0.651287048
C -3.830556273
$-0.216633016$
$-0.845859061$
$\begin{array}{lll}\text { C } & -4.365533315 & -1.330585098\end{array}$
$-0.195822015$
C $\quad 0.028662002$
1.212328086
0.461213036
C $\quad-0.630727046$
2.034235145
1.392501101
C $\quad 0.929961069$
1.786946129
$-0.441234033$
C $\quad-0.367966025$
3.397525244
1.419194103
C $\quad 1.176309086$
3.158129229
$-0.405834028$
C $\quad 0.532062038$
3.954936285
0.516519038
C $\quad-0.268981018$
$-0.251633016$
0.389242030
H $\quad-1.622261115$
$-2.324934169$
1.503474111
$\mathrm{H} \quad-2.101863151$
0.999638070
$-1.138553083$
H $\quad-3.962444283$
$-2.936192212$
1.172639083
H $\quad-4.445592319$
0.376940030
$-1.496623107$
H $\quad-5.395673389$
$-1.599208117$
$-0.343334025$
H $\quad-1.314926092$
1.601517113
2.097254149
H 1.419948102
1.174222085
$-1.176900087$
H $\quad-0.864001061$
4.023561292
2.137513155
H $\quad 1.868492137$
3.591540261
$-1.104577078$
H $\quad 0.723771054$
5.012981362
0.536940041
C $\quad 0.741693054$
$-1.154731084$
0.455984034
C $\quad 2.130642154$
$-0.797395056$
0.907369063 


$\begin{array}{lrrr}\mathrm{O} & 2.364699172 & -0.149502013 & 1.876660136 \\ \mathrm{O} & 3.052242222 & -1.331833094 & 0.143662008 \\ \mathrm{C} & 4.403694319 & -1.171757082 & 0.552450041 \\ \mathrm{H} & 4.672502338 & -0.125818008 & 0.555008042 \\ \mathrm{H} & 4.990962360 & -1.712453123 & -0.171699015 \\ \mathrm{H} & 4.546072326 & -1.585268112 & 1.539735108 \\ \mathrm{C} & 0.584022042 & -2.549466181 & 0.116858011 \\ \mathrm{~N} & 0.530946040 & -3.648670263 & -0.160241009 \\ \mathrm{C} & 1.110233078 & -0.338306026 & 5.230317374 \\ \mathrm{O} & 0.458505035 & 0.307693022 & 4.178332302 \\ \mathrm{H} & 1.046195074 & 0.346939024 & 3.433872249 \\ \mathrm{H} & 1.367134101 & -1.366339097 & 4.982752360 \\ \mathrm{H} & 2.019233144 & 0.179870014 & 5.528219400 \\ \mathrm{H} & 0.436779033 & -0.353304026 & 6.076412437\end{array}$

\section{Coord_2}

\begin{tabular}{|c|c|c|}
\hline-1.675295122 & -0.625745043 & 01 \\
\hline-2.202724160 & -1.765108127 & 5057 \\
\hline-2.524388181 & 0.171606012 & -0.5846250 \\
\hline-3.539998254 & -2.115568153 & 0.6350300 \\
\hline-3.856546279 & -0.184595011 & \\
\hline-4.370789315 & -1.332731097 & -0.1662 \\
\hline 0.028143001 & 1.229412086 & 0.4516240 \\
\hline-0.624844045 & 2.054362147 & 1.383692099 \\
\hline 0.933813066 & 1.800738128 & -0.448820033 \\
\hline-0.356278023 & 3.417423245 & 1.410 \\
\hline 1.187557087 & 3.168541226 & -0.413192 \\
\hline 0.546637040 & 3.970809284 & 0.509219038 \\
\hline-0.271854022 & -0.233540017 & 0.3841040 \\
\hline-1.585954116 & -2.367361171 & 1.449 \\
\hline-2.142515155 & 1.058041077 & -1.054102077 \\
\hline-3.925190284 & -2.990209218 & 1.125677079 \\
\hline 4325 & 0.429685031 & -1.39220 \\
\hline-5.399250390 & -1.607856117 & -0.31108302 \\
\hline-1.307410093 & 1.624491116 & 2.09194015 \\
\hline 1.422335104 & 1.185386084 & -1.183257083 \\
\hline-0.848744061 & 4.044798291 & 2.1305 \\
\hline $1.883^{3}$ & 3.597902257 & -1.110 \\
\hline 0.745178051 & 5.027538361 & 0.530135039 \\
\hline 0.751361054 & -1.139171082 & 0.435932032 \\
\hline 2.127245153 & -0.786127057 & 0.91664806 \\
\hline 170 & -0.122040011 & $1.8 \varepsilon$ \\
\hline 3.065816222 & -1.336104094 & 0.1833370 \\
\hline 4.407863315 & -1.165486083 & 0.6156320 \\
\hline 4.675271337 & -0.119181009 & 0.6032150 \\
\hline 5.01058 & -1.718886124 & -0.08600 \\
\hline & & \\
\hline
\end{tabular}




$\begin{array}{rrrr}\mathrm{C} & 0.603567045 & -2.516707183 & 0.037623005 \\ \mathrm{~N} & 0.543037041 & -3.604713261 & -0.281786023 \\ \mathrm{C} & 1.104253078 & -0.309207024 & 5.225137374 \\ \mathrm{O} & 0.442195030 & 0.331650024 & 4.176581302 \\ \mathrm{H} & 1.024903072 & 0.371688025 & 3.428058247 \\ \mathrm{H} & 1.366287100 & -1.335807099 & 4.976933358 \\ \mathrm{H} & 2.011769147 & 0.214901013 & 5.517158399 \\ \mathrm{H} & 0.436076032 & -0.327795026 & 6.075406440\end{array}$

Coord_3

$\begin{array}{lrrr}\mathrm{C} & -1.668815119 & -0.613513042 & 0.212053013 \\ \mathrm{C} & -2.180553157 & -1.787266128 & 0.790413059 \\ \mathrm{C} & -2.547449185 & 0.204175014 & -0.522837039 \\ \mathrm{C} & -3.521163253 & -2.144392152 & 0.618407043 \\ \mathrm{C} & -3.882517277 & -0.158931010 & -0.705542051 \\ \mathrm{C} & -4.375956313 & -1.338820096 & -0.135655011 \\ \mathrm{C} & 0.027143004 & 1.244223091 & 0.439922034 \\ \mathrm{C} & -0.619110044 & 2.072670151 & 1.372538101 \\ \mathrm{C} & 0.936913066 & 1.811728129 & -0.459088035 \\ \mathrm{C} & -0.344677024 & 3.435480249 & 1.399358101 \\ \mathrm{C} & 1.198086084 & 3.176119229 & -0.424013029 \\ \mathrm{C} & 0.560848042 & 3.984332287 & 0.498237038 \\ \mathrm{C} & -0.275229019 & -0.217732016 & 0.377614027 \\ \mathrm{H} & -1.549368114 & -2.408695175 & 1.393751102 \\ \mathrm{H} & -2.183489159 & 1.109338080 & -0.969258068 \\ \mathrm{H} & -3.887774278 & -3.043360220 & 1.077935078 \\ \mathrm{H} & -4.527966325 & 0.473983035 & -1.285242090 \\ \mathrm{H} & -5.402757390 & -1.620485117 & -0.277228018 \\ \mathrm{H} & -1.299807091 & 1.646014120 & 2.084696148 \\ \mathrm{H} & 1.423706101 & 1.193350085 & -1.192120087 \\ \mathrm{H} & -0.833326059 & 4.064559294 & 2.120287155 \\ \mathrm{H} & 1.897187138 & 3.601020260 & -1.121149078 \\ \mathrm{H} & 0.766257055 & 5.039703363 & 0.519046039 \\ \mathrm{C} & 0.760162055 & -1.126053080 & 0.414601029 \\ \mathrm{C} & 2.122543154 & -0.779141055 & 0.924729067 \\ \mathrm{O} & 2.324608169 & -0.099998006 & 1.882837137 \\ \mathrm{O} & 3.076515222 & -1.345302094 & 0.222256016 \\ \mathrm{C} & 4.408958315 & -1.166265085 & 0.677829049 \\ \mathrm{H} & 4.677082335 & -0.120335007 & 0.650988047 \\ \mathrm{H} & 5.025766364 & -1.732608124 & -0.000999002 \\ \mathrm{H} & 4.514029326 & -1.540625111 & 1.685373122 \\ \mathrm{C} & 0.622140044 & -2.483948180 & -0.041613001 \\ \mathrm{~N} & 0.555175039 & -3.559072257 & -0.403078031 \\ \mathrm{C} & 1.096726077 & -0.286531023 & 5.218456374 \\ \mathrm{O} & 0.425169032 & 0.350291023 & 4.173611300 \\ \mathrm{H} & 1.003019073 & 0.390898027 & 3.421161247 \\ \mathrm{H} & 1.362923099 & -1.311800094 & 4.969113357\end{array}$




$\begin{array}{rrrr}\mathrm{H} & 2.003157142 & 0.242473016 & 5.505000398 \\ \mathrm{H} & 0.433726030 & -0.308453021 & 6.072758437\end{array}$

Coord_4

\begin{tabular}{|c|c|c|c|}
\hline & -1.662149121 & -0.604123046 & 587015 \\
\hline & -2.158051154 & -1.810237130 & 986054 \\
\hline & -2.570316183 & 0.231032019 & -0.460900033 \\
\hline & -3.501993252 & -2.174380156 & 0.601358042 \\
\hline & -3.908147279 & -0.139661010 & -0.633418044 \\
\hline & -4.380733313 & -1.348950099 & -0.104217009 \\
\hline & 0.025815002 & 1.256885090 & 0.425938 \\
\hline & -0.613381044 & 2.089289153 & 1.358844100 \\
\hline & 0.939428066 & 1.820031132 & -0.472199036 \\
\hline & -0.333019022 & 3.451825248 & 1.384547101 \\
\hline & 1.208060086 & 3.180978230 & -0.438466029 \\
\hline & 0.574851040 & 3.995627290 & 0.483375037 \\
\hline & -0.278944018 & -0.204083016 & 0.369607025 \\
\hline & -1.512369109 & -2.448847176 & 1.335185098 \\
\hline & -2.224548160 & 1.153684081 & -0.884350065 \\
\hline & -3.849979280 & -3.095773221 & 1.029448074 \\
\hline & -4.568825328 & 0.509793037 & -1.176117084 \\
\hline & -5.405864391 & -1.637277118 & -0.241933017 \\
\hline & -1.291975093 & 1.666228119 & 2.075317148 \\
\hline & 1.424233104 & 1.198224088 & -1.203636085 \\
\hline & -0.817607060 & 4.082982293 & 2.106506152 \\
\hline & 1.910295139 & 3.601001257 & -1.135437081 \\
\hline & 0.787 & 5.0 & 035 \\
\hline & 0.768258054 & -1.115268083 & 0.391865029 \\
\hline & 2.116667154 & -0.776321053 & 0.931445067 \\
\hline & 2.303775164 & -0.083181007 & 1.884030135 \\
\hline & 3.084400223 & -1.359377098 & 0.260213017 \\
\hline & 4.407073317 & -1.174012086 & 0.738865052 \\
\hline & 4.677999336 & -0.129173008 & 0.698199050 \\
\hline & 5.036547361 & -1.753599125 & 0.083096005 \\
\hline & 4.492151323 & -1.529575112 & 1.755151128 \\
\hline & 0.639909044 & -2.451189177 & -0.120848007 \\
\hline & 0.567381042 & -3.511762250 & -0.524020036 \\
\hline & 1.087951080 & -0.270087020 & 5.210186376 \\
\hline & 0.407674030 & 0.363799024 & 4.169328298 \\
\hline & 0.980773070 & 0.404775028 & 3.413070244 \\
\hline & 1.357359096 & -1.294138095 & 4.9592163 \\
\hline & 1.993708142 & 0.262811018 & 5.491632397 \\
\hline & 0.430035029 & -0.295106022 & 6.06 \\
\hline
\end{tabular}

Coord_5
C $\quad-1.655164118 \quad-0.597402045$
0.239703017
$\begin{array}{llll}\text { C } & -2.135087153 & -1.833940133 & 0.741950054\end{array}$ 


$\begin{array}{lrrr}\text { C } & -2.592787187 & 0.252304018 & -0.399064031 \\ \text { C } & -3.482301249 & -2.205573160 & 0.583825040 \\ \text { C } & -3.933168282 & -0.126760007 & -0.560348038 \\ \text { C } & -4.384863314 & -1.363181099 & -0.072096003 \\ \text { C } & 0.024273001 & 1.267524093 & 0.409533030 \\ \text { C } & -0.607537043 & 2.104353152 & 1.342455094 \\ \text { C } & 0.941485068 & 1.825763132 & -0.488284035 \\ \text { C } & -0.321171024 & 3.466588251 & 1.366245099 \\ \text { C } & 1.217624086 & 3.183225228 & -0.456684035 \\ \text { C } & 0.588793040 & 4.004810288 & 0.464484034 \\ \text { C } & -0.282894019 & -0.192454013 & 0.359945026 \\ \text { H } & -1.474870106 & -2.487717181 & 1.274171094 \\ \text { H } & -2.265516163 & 1.191267085 & -0.799653055 \\ \text { H } & -3.811632276 & -3.147553225 & 0.980239072 \\ \text { H } & -4.609027331 & 0.537124036 & -1.065177074 \\ \text { H } & -5.408285387 & -1.658370118 & -0.205351015 \\ \text { H } & -1.283802093 & 1.685282120 & 2.063634148 \\ \text { H } & 1.424044103 & 1.200120088 & -1.217922087 \\ \text { H } & -0.801450056 & 4.100203298 & 2.089032153 \\ \text { H } & 1.922787141 & 3.597946258 & -1.153901080 \\ \text { H } & 0.808062057 & 5.057334364 & 0.483241034 \\ \text { C } & 0.775751055 & -1.106703079 & 0.367617027 \\ \text { C } & 2.109689153 & -0.777540055 & 0.936639069 \\ \text { O } & 2.282601163 & -0.071391006 & 1.883771135 \\ \text { O } & 3.089466222 & -1.378261100 & 0.296998022 \\ \text { C } & 4.402226318 & -1.188629084 & 0.798555060 \\ \text { H } & 4.678007337 & -0.145579011 & 0.744709052 \\ \text { H } & 5.042897361 & -1.781813131 & 0.166058012 \\ \text { H } & 4.466552321 & -1.525848111 & 1.822670134 \\ \text { C } & 0.656983048 & -2.418429173 & -0.200083013 \\ \text { N } & 0.579673041 & -3.462805248 & -0.644515045 \\ \text { C } & 1.078159078 & -0.259690020 & 5.200240373 \\ \text { O } & 0.389891029 & 0.372352026 & 4.163649300 \\ \text { H } & 0.958326070 & 0.413517031 & 3.403687243 \\ \text { H } & 1.349845097 & -1.282638094 & 4.947164354 \\ \text { H } & 1.983663141 & 0.276132018 & 5.476942394 \\ \text { H } & 0.425249028 & -0.287592023 & 6.062243436\end{array}$

Coord_6

$\begin{array}{lrrr}C & -1.647773121 & -0.593162044 & 0.251258020 \\ C & -2.111566150 & -1.858279132 & 0.715246051 \\ C & -2.614702190 & 0.268145018 & -0.337546022 \\ C & -3.461932248 & -2.237997162 & 0.565752038 \\ C & -3.957352286 & -0.120178011 & -0.486563034 \\ C & -4.388124315 & -1.381549101 & -0.039441004 \\ C & 0.022608003 & 1.276267091 & 0.390592030 \\ C & -0.601475043 & 2.117999151 & 1.323243097\end{array}$




\begin{tabular}{|c|c|c|c|}
\hline & 36 & 90 & \\
\hline C & .308998021 & 3.479894249 & \\
\hline C & 26907088 & 3.182965227 & -0.4787640 \\
\hline 0 & 0.602819042 & 4.011992287 & 0.44145203 \\
\hline & -0.287013018 & -0.182701012 & 0.34850602 \\
\hline & -1.436819103 & -2.525199182 & 1.21012 \\
\hline & 265166 & 1.222295086 & \\
\hline & -3.772581270 & -3.198793231 & 0.9303150 \\
\hline & -4.648298337 & 0.556017040 & -0.9527220 \\
\hline & 765390 & 878123 & \\
\hline & -1.275189093 & 1.703331123 & 2.04951 \\
\hline & 1.423231101 & 1.199146085 & -1.2350770 \\
\hline & -0.784710058 & 4.116357296 & 2.067736150 \\
\hline & 1.934803137 & 3.591944256 & -1.176630085 \\
\hline & 0.829112061 & 5.06301 & 0.458262 \\
\hline 乙 & 0.782701058 & -1.100240078 & 0.341761 \\
\hline U & 2.101629154 & -0.782663057 & 0.94016306 \\
\hline & 2.261157160 & -0.064425007 & 1.8819301 \\
\hline & 3.091 & -1.4 & 0.3 \\
\hline C & 4.394377317 & -1.209995086 & 0.856702 \\
\hline$\Pi$ & 4.677023337 & -0.169427013 & 0.790364058 \\
\hline & 5.04 & & \\
\hline & & & \\
\hline$C$ & 0.67 & -2.38 & -0.2 \\
\hline$N$ & 0.592065044 & -3.412229243 & -0.7644660 \\
\hline 乙 & 1.067 & -0.25 & 5.1 \\
\hline 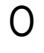 & & & \\
\hline & 0.93 & 0.41 & 3.3 \\
\hline & 1.340583098 & -1.27 & 4.932 \\
\hline $\mathrm{H}_{\text {- }}$ & 1.973207 & 0.282656 & 5.4608 \\
\hline & & & \\
\hline \multicolumn{4}{|c|}{ Coo } \\
\hline 0 & -1.639 & -0.59 & 0.26 \\
\hline C & & & \\
\hline$C$ & -2.63 & $0.27 \varepsilon$ & -0.2 \\
\hline C & -3.440747246 & -2.271667165 & 0.5 \\
\hline C & -3.980499287 & -0.119853011 & -0.4122610 \\
\hline C & -4.390315316 & -1.404082103 & -0.0063819 \\
\hline C & 0.0208 & & \\
\hline C & & 2.13 & 1.3 \\
\hline C & 0.94 & 1.829958132 & -0.52977403 \\
\hline C & -0.296360019 & 3.491870250 & 1.3186960 \\
\hline 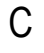 & 1.23603 & 3.180287230 & -0.50478 \\
\hline C & & 4.017272290 & \\
\hline C & -0.2 & -0.174673012 & $0.3351 \varepsilon$ \\
\hline & & & 1.144 \\
\hline
\end{tabular}




$\begin{array}{rrrr}\mathrm{H} & -2.346709167 & 1.246987089 & -0.631748046 \\ \mathrm{H} & -3.732688269 & -3.249574233 & 0.879669063 \\ \mathrm{H} & -4.686398337 & 0.566527040 & -0.839007062 \\ \mathrm{H} & -5.410062391 & -1.713904121 & -0.128884008 \\ \mathrm{H} & -1.266042090 & 1.720539126 & 2.032834148 \\ \mathrm{H} & 1.421858103 & 1.195404087 & -1.255185093 \\ \mathrm{H} & -0.767223057 & 4.131579296 & 2.042519145 \\ \mathrm{H} & 1.946477141 & 3.583067260 & -1.203694088 \\ \mathrm{H} & 0.850502062 & 5.066726366 & 0.428446031 \\ \mathrm{C} & 0.789131059 & -1.095756079 & 0.314211025 \\ \mathrm{C} & 2.092474149 & -0.791548055 & 0.941873066 \\ \mathrm{O} & 2.239471159 & -0.062077005 & 1.878375136 \\ \mathrm{O} & 3.090874225 & -1.430103103 & 0.366220026 \\ \mathrm{C} & 4.383438313 & -1.237972089 & 0.913101064 \\ \mathrm{H} & 4.674915339 & -0.200584012 & 0.834993062 \\ \mathrm{H} & 5.041876361 & -1.859586136 & 0.327601025 \\ \mathrm{H} & 4.404405316 & -1.539651112 & 1.950189140 \\ \mathrm{C} & 0.689298051 & -2.352911172 & -0.358554025 \\ \mathrm{~N} & 0.604569042 & -3.360068242 & -0.883780061 \\ \mathrm{C} & 1.056208076 & -0.256284018 & 5.174983373 \\ \mathrm{O} & 0.353945024 & 0.375293029 & 4.147798298 \\ \mathrm{H} & 0.913238068 & 0.416389029 & 3.380669244 \\ \mathrm{H} & 1.329735097 & -1.277367092 & 4.916282353 \\ \mathrm{H} & 1.962479144 & 0.282605020 & 5.443153390 \\ \mathrm{H} & 0.413135028 & -0.289413022 & 6.044305433\end{array}$

\section{Coord_8}

$\begin{array}{rrrr}\text { C } & -1.631595119 & -0.591293043 & 0.269222021 \\ \text { C } & -2.062583149 & -1.908432139 & 0.656627049 \\ \text { C } & -2.656416190 & 0.284199018 & -0.216143014 \\ \text { C } & -3.418614248 & -2.306596166 & 0.527752037 \\ \text { C } & -4.002435287 & -0.125722010 & -0.337608026 \\ \text { C } & -4.391246318 & -1.430805104 & 0.026960004 \\ \text { C } & 0.019177002 & 1.288592095 & 0.344742023 \\ \text { C } & -0.588288041 & 2.141604156 & 1.275971090 \\ \text { C } & 0.945854066 & 1.828625129 & -0.555339042 \\ \text { C } & -0.283099022 & 3.502636250 & 1.289281094 \\ \text { C } & 1.245132090 & 3.175268227 & -0.534806039 \\ \text { C } & 0.631757047 & 4.020740290 & 0.382642026 \\ \text { C } & -0.295667019 & -0.168213012 & 0.319894024 \\ \text { H } & -1.358942098 & -2.595532188 & 1.076536076 \\ \text { H } & -2.386806170 & 1.265564089 & -0.548845037 \\ \text { H } & -3.691810264 & -3.299975239 & 0.828280057 \\ \text { H } & -4.723115337 & 0.568705043 & -0.724244054 \\ \text { H } & -5.408943387 & -1.748552123 & -0.089249008 \\ \text { H } & -1.256263090 & 1.737075126 & 2.013516146 \\ \text { H } & 1.419964104 & 1.188983087 & -1.278319092\end{array}$




$\begin{array}{rrrr}\mathrm{H} & -0.748796052 & 4.146001297 & 2.013310143 \\ \mathrm{H} & 1.957944142 & 3.571367257 & -1.235148087 \\ \mathrm{H} & 0.872443063 & 5.068542364 & 0.393736031 \\ \mathrm{C} & 0.795036058 & -1.093130079 & 0.284895018 \\ \mathrm{C} & 2.082176148 & -0.804046057 & 0.941629067 \\ \mathrm{O} & 2.217535159 & -0.064135002 & 1.872982137 \\ \mathrm{O} & 3.086988222 & -1.462841103 & 0.398236027 \\ \mathrm{C} & 4.369283314 & -1.272403092 & 0.967536072 \\ \mathrm{H} & 4.671501336 & -0.238904017 & 0.878414061 \\ \mathrm{H} & 5.034179361 & -1.908916135 & 0.405659029 \\ \mathrm{H} & 4.367857314 & -1.556765112 & 2.009796147 \\ \mathrm{C} & 0.704584050 & -2.320151168 & -0.437790031 \\ \mathrm{~N} & 0.617194047 & -3.306366239 & -1.002365074 \\ \mathrm{C} & 1.044291073 & -0.262889018 & 5.159511373 \\ \mathrm{O} & 0.335926025 & 0.370030028 & 4.137488296 \\ \mathrm{H} & 0.890694064 & 0.410917030 & 3.366862243 \\ \mathrm{H} & 1.317427095 & -1.283199091 & 4.897298352 \\ \mathrm{H} & 1.951580140 & 0.276207018 & 5.423832393 \\ \mathrm{H} & 0.406089028 & -0.298416021 & 6.032388436\end{array}$

Coord_9

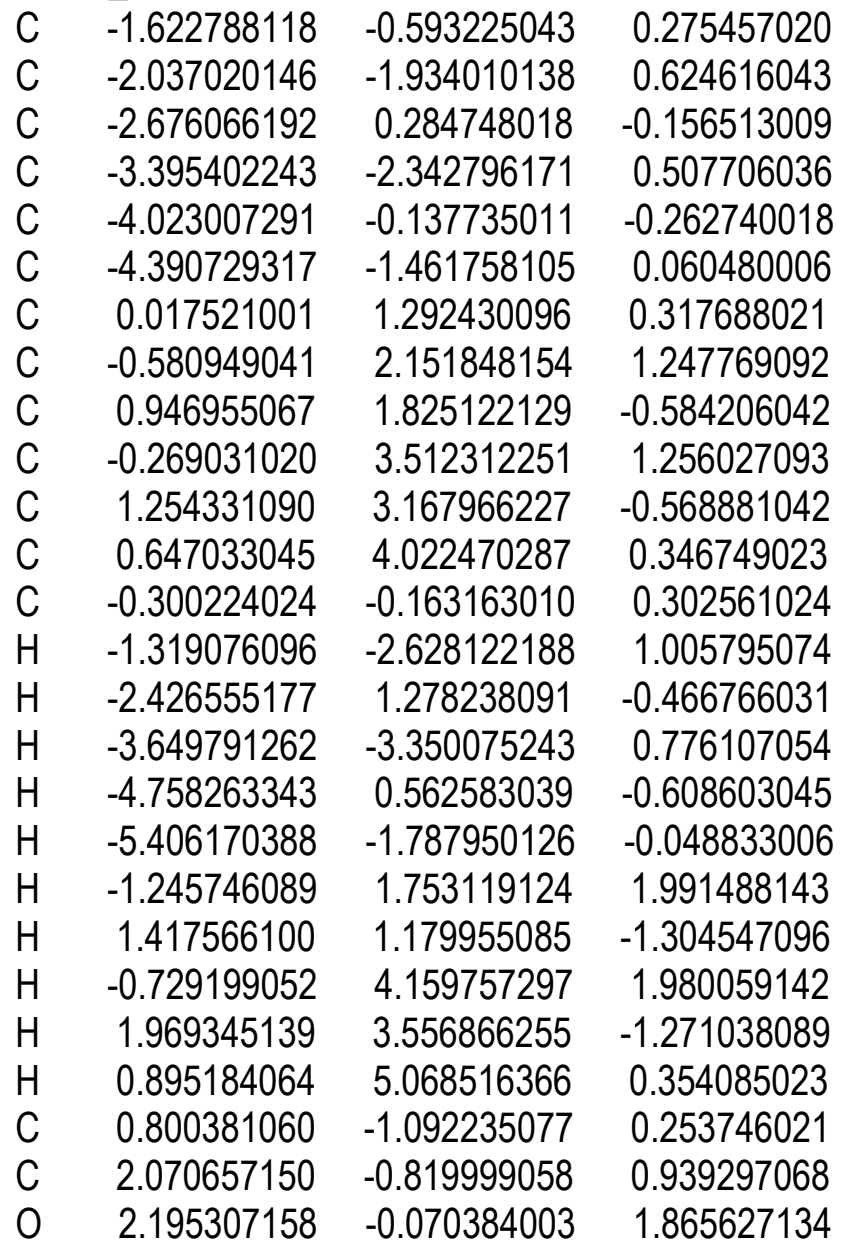




$\begin{array}{lrrr}\text { O } & 3.079835222 & -1.499950108 & 0.428241033 \\ \mathrm{C} & 4.351744311 & -1.313112094 & 1.019781075 \\ \mathrm{H} & 4.666558333 & -0.284238018 & 0.920426067 \\ \mathrm{H} & 5.021406361 & -1.965012141 & 0.481536035 \\ \mathrm{H} & 4.327600313 & -1.580371116 & 2.066359151 \\ \mathrm{C} & 0.719273052 & -2.287392165 & -0.517025037 \\ \mathrm{~N} & 0.629947044 & -3.251175232 & -1.120136079 \\ \mathrm{C} & 1.031854074 & -0.274766021 & 5.142035368 \\ \mathrm{O} & 0.317918023 & 0.360515024 & 4.125504299 \\ \mathrm{H} & 0.868158061 & 0.401107031 & 3.351414241 \\ \mathrm{H} & 1.303757092 & -1.294394095 & 4.875850349 \\ \mathrm{H} & 1.940575137 & 0.263698019 & 5.402740388 \\ \mathrm{H} & 0.398519031 & -0.312581022 & 6.018424432 \\ & & & \\ \mathrm{Coord} & 10 & & \\ \mathrm{C} & -1.603197113 & -0.672395050 & 0.332472022 \\ \mathrm{C} & -1.972212142 & -2.038350149 & 0.664399049 \\ \mathrm{C} & -2.703012194 & 0.177922012 & -0.053212006 \\ \mathrm{C} & -3.324298241 & -2.487036178 & 0.571898041 \\ \mathrm{C} & -4.041665292 & -0.286158021 & -0.131722007 \\ \mathrm{C} & -4.360582312 & -1.629680117 & 0.170568015 \\ \mathrm{C} & -0.011343999 & 1.250889089 & 0.307118023 \\ \mathrm{C} & -0.613848043 & 2.118705153 & 1.225637089 \\ \mathrm{C} & 0.906811067 & 1.780759126 & -0.608173046 \\ \mathrm{C} & -0.321741022 & 3.484377248 & 1.207437085 \\ \mathrm{C} & 1.196146083 & 3.125609224 & -0.619126046 \\ \mathrm{C} & 0.581911042 & 3.990722288 & 0.284353019 \\ \mathrm{C} & -0.303546024 & -0.209310018 & 0.324431025 \\ \mathrm{H} & -1.225185087 & -2.717867194 & 1.013297071 \\ \mathrm{H} & -2.495087181 & 1.182688085 & -0.356237028 \\ \mathrm{H} & -3.538735256 & -3.507654253 & 0.823549059 \\ \mathrm{H} & -4.806134347 & 0.398530027 & -0.443216030 \\ \mathrm{H} & -5.367050388 & -1.986237144 & 0.077530007 \\ \mathrm{H} & -1.264638093 & 1.724158126 & 1.984000142 \\ \mathrm{H} & 1.383630101 & 1.128715080 & -1.318155095 \\ \mathrm{H} & -0.786084056 & 4.137581299 & 1.923693140 \\ \mathrm{H} & 1.902950138 & 3.510662255 & -1.331666096 \\ \mathrm{H} & 0.817138061 & 5.039649361 & 0.271447021 \\ \mathrm{C} & 0.824532059 & -1.121655083 & 0.270951018 \\ \mathrm{C} & 2.074587148 & -0.833130062 & 0.976358071 \\ \mathrm{O} & 2.177864159 & -0.059252003 & 1.886429134 \\ \mathrm{O} & 3.097904223 & -1.521904111 & 0.503701037 \\ \mathrm{C} & 4.357717314 & -1.306958096 & 1.109655078 \\ \mathrm{H} & 4.666246336 & -0.278856021 & 0.984993068 \\ \mathrm{H} & 5.041559362 & -1.968681143 & 0.601916043 \\ \mathrm{H} & 4.319437310 & -1.543068110 & 2.163358156 \\ \mathrm{C} & 0.772707053 & -2.296117165 & -0.529088040 \\ & & & \end{array}$




$\begin{array}{rrrr}\mathrm{N} & 0.698458051 & -3.246720232 & -1.156015085 \\ \mathrm{C} & 1.040448077 & -0.246208018 & 5.159919369 \\ \mathrm{O} & 0.305262024 & 0.363596026 & 4.143071296 \\ \mathrm{H} & 0.847189063 & 0.403424028 & 3.362891244 \\ \mathrm{H} & 1.329375097 & -1.263494091 & 4.902794355 \\ \mathrm{H} & 1.941160138 & 0.312271024 & 5.405929390 \\ \mathrm{H} & 0.416142031 & -0.285983023 & 6.042743434\end{array}$

LIIC path connecting the $V\left({ }^{1} \pi \pi^{*}\right) / T_{2}\left({ }^{3} \pi \pi^{*}\right)$ crossing point and $T_{2}\left({ }^{3} \pi \pi^{*}\right)$ minimum Coord_1

\begin{tabular}{|c|c|c|c|}
\hline & -1.603197113 & -0.672395050 & 72 \\
\hline & -1.972212142 & -2.038350149 & 0.664399049 \\
\hline & -2.703012194 & 0.177922012 & -0.053212006 \\
\hline & -3.324298241 & -2.487036178 & 0.571898041 \\
\hline & -4.041665292 & -0.286158021 & -0.131722007 \\
\hline & -4.360582312 & -1.629680117 & $0.1 / 05000$ \\
\hline & -0.011343999 & 1.250889089 & 0.307118023 \\
\hline & -0.613848043 & 2.118705153 & 1.225637089 \\
\hline & 0.906811067 & 1.780759126 & -0.608173046 \\
\hline & -0.321741022 & 3.484377248 & 1.207437085 \\
\hline & 1.196146083 & 3.125609224 & -0.619126 \\
\hline & 0.581911042 & 3.990722288 & 0.284353019 \\
\hline & -0.303546024 & -0.209310018 & 0.324431025 \\
\hline & -1.225185087 & -2.717867194 & 1.013297071 \\
\hline & -2.495087181 & 1.182688085 & -0.356237028 \\
\hline & -3.538735256 & -3.507654253 & 0.823549059 \\
\hline & -4.806134347 & 0.398530027 & -0.443216030 \\
\hline & -5.367050388 & -1.986237144 & 0.077530007 \\
\hline & -1.264638093 & 1.724158126 & 1.984000142 \\
\hline & 1.383630101 & 1.128715080 & -1.318155095 \\
\hline & -0.786084056 & 4.137581299 & 1.923693140 \\
\hline & 1.902950138 & 3.510662255 & -1.331666096 \\
\hline & 38061 & 49361 & 0.271447021 \\
\hline & 0.824532059 & -1.121655083 & 0.270951018 \\
\hline & 2.074587148 & -0.833130062 & 0.976358071 \\
\hline & 2.177864159 & -0.059252003 & 1.886429134 \\
\hline & 3.097904223 & -1.521904111 & 0.5037 \\
\hline & 4.357717314 & -1.306958096 & 1.109655078 \\
\hline & 4.666246336 & -0.278856021 & 0.984993068 \\
\hline & 5.041559362 & -1.968681143 & 0.601916043 \\
\hline & 4.319437310 & -1.543068110 & 2.163358156 \\
\hline & 0.772707053 & -2.296117165 & -0.529088040 \\
\hline & 0.698458051 & -3.246720232 & -1.156015085 \\
\hline & 1.040448077 & -0.246208018 & 5.159919369 \\
\hline & 0.3052 & 0.363596026 & 4.14307 \\
\hline & 0.847189063 & 0.403 & $3.362 \varepsilon$ \\
\hline & 1.329375097 & -1.263 & 4.9027 \\
\hline
\end{tabular}




$\begin{array}{rrrr}H & 1.941160138 & 0.312271024 & 5.405929390 \\ \mathrm{H} & 0.416142031 & -0.285983023 & 6.042743434\end{array}$

Coord_2

$\begin{array}{lrrr}\text { C } & -1.616176117 & -0.672462049 & 0.331300026 \\ \mathrm{C} & -2.000338146 & -2.020644148 & 0.694292050 \\ \mathrm{C} & -2.693143192 & 0.178054015 & -0.101397007 \\ \mathrm{C} & -3.348372239 & -2.459044178 & 0.589952044 \\ \mathrm{C} & -4.031084293 & -0.275708020 & -0.194711012 \\ \mathrm{C} & -4.366821312 & -1.604469118 & 0.142005011 \\ \mathrm{C} & -0.012065003 & 1.246029088 & 0.337250027 \\ \mathrm{C} & -0.612011047 & 2.106625153 & 1.259455093 \\ \mathrm{C} & 0.904177066 & 1.782584126 & -0.586368043 \\ \mathrm{C} & -0.321976024 & 3.478628251 & 1.246725088 \\ \mathrm{C} & 1.195469086 & 3.137179224 & -0.587276043 \\ \mathrm{C} & 0.586150043 & 3.991607289 & 0.318669021 \\ \mathrm{C} & -0.303751022 & -0.215614016 & 0.346391024 \\ \mathrm{H} & -1.265245091 & -2.695656196 & 1.077090077 \\ \mathrm{H} & -2.468273177 & 1.173992083 & -0.422360033 \\ \mathrm{H} & -3.578990256 & -3.469206250 & 0.868945064 \\ \mathrm{H} & -4.784140343 & 0.404781029 & -0.541869041 \\ \mathrm{H} & -5.375202385 & -1.954669138 & 0.042289004 \\ \mathrm{H} & -1.262554092 & 1.708874122 & 2.016211145 \\ \mathrm{H} & 1.371290100 & 1.136766083 & -1.307913092 \\ \mathrm{H} & -0.787404057 & 4.128896299 & 1.964303144 \\ \mathrm{H} & 1.901096139 & 3.525328254 & -1.299291096 \\ \mathrm{H} & 0.820093061 & 5.040888360 & 0.313285023 \\ \mathrm{C} & 0.818737060 & -1.124416081 & 0.305303025 \\ \mathrm{C} & 2.082388152 & -0.812445058 & 0.984333073 \\ \mathrm{O} & 2.190739157 & -0.041059000 & 1.894762136 \\ \mathrm{O} & 3.105471225 & -1.481683105 & 0.486735038 \\ \mathrm{C} & 4.373903317 & -1.254205088 & 1.071276075 \\ \mathrm{H} & 4.664844334 & -0.220548017 & 0.951040067 \\ \mathrm{H} & 5.057223363 & -1.901038136 & 0.544193040 \\ \mathrm{H} & 4.358158315 & -1.501395106 & 2.122950155 \\ \mathrm{C} & 0.760983057 & -2.328240166 & -0.453299031 \\ \mathrm{~N} & 0.687160047 & -3.299634237 & -1.046396076 \\ \mathrm{C} & 1.047488076 & -0.231052016 & 5.178902375 \\ \mathrm{O} & 0.313469023 & 0.374059025 & 4.158308298 \\ \mathrm{H} & 0.858218064 & 0.417237031 & 3.380458246 \\ \mathrm{H} & 1.341890097 & -1.247405092 & 4.924358355 \\ \mathrm{H} & 1.944634139 & 0.331907025 & 5.427658392 \\ \mathrm{H} & 0.419800032 & -0.272359021 & 6.059187437\end{array}$

Coord_3
C $\quad-1.628576116 \quad-0.673926049$
0.327720026
$\begin{array}{llll}\text { C } & -2.027970147 & -2.003224146 & 0.721834053\end{array}$ 


$\begin{array}{lrrr}\text { C } & -2.682015192 & 0.174641013 & -0.151621008 \\ \text { C } & -3.371594241 & -2.432042173 & 0.606065042 \\ \text { C } & -4.018762289 & -0.269633017 & -0.259407016 \\ \text { C } & -4.371554315 & -1.582296115 & 0.111716006 \\ \text { C } & -0.013115002 & 1.240352090 & 0.364853029 \\ \text { C } & -0.610697047 & 2.093949151 & 1.290339094 \\ \text { C } & 0.901066065 & 1.783476128 & -0.567226040 \\ \text { C } & -0.322998024 & 3.472306250 & 1.282652091 \\ \text { C } & 1.194142084 & 3.147779226 & -0.558438041 \\ \text { C } & 0.589548044 & 3.991776287 & 0.349507026 \\ \text { C } & -0.303989019 & -0.222752014 & 0.366272028 \\ \text { H } & -1.305204096 & -2.672273192 & 1.138410083 \\ \text { H } & -2.440352177 & 1.161075084 & -0.490569038 \\ \text { H } & -3.618600258 & -3.430595245 & 0.912343065 \\ \text { H } & -4.759953345 & 0.405151030 & -0.642050047 \\ \text { H } & -5.381669386 & -1.926462139 & 0.005501999 \\ \text { H } & -1.260957088 & 1.693164120 & 2.045593146 \\ \text { H } & 1.358532097 & 1.143823080 & -1.299881091 \\ \text { H } & -0.789657056 & 4.119810299 & 2.001287146 \\ \text { H } & 1.898573138 & 3.538859253 & -1.270020090 \\ \text { H } & 0.822047058 & 5.041392365 & 0.351287025 \\ \text { C } & 0.812396056 & -1.128403082 & 0.338121026 \\ \text { C } & 2.089442149 & -0.795086060 & 0.989963069 \\ \text { O } & 2.204430158 & -0.026717000 & 1.900930136 \\ \text { O } & 3.110104224 & -1.446104105 & 0.466709034 \\ \text { C } & 4.387339318 & -1.208224088 & 1.029098077 \\ \text { H } & 4.662425337 & -0.169738011 & 0.913978064 \\ \text { H } & 5.068117366 & -1.840924135 & 0.482121035 \\ \text { H } & 4.394457318 & -1.466643104 & 2.078099150 \\ \text { C } & 0.748712056 & -2.360362167 & -0.377511028 \\ \text { N } & 0.675949046 & -3.351481240 & -0.935929066 \\ \mathrm{C} & 1.055857078 & -0.219881016 & 5.195960372 \\ \text { O } & 0.323641022 & 0.382014030 & 4.172071299 \\ \text { H } & 0.871043061 & 0.428111030 & 3.396437245 \\ \text { H } & 1.354216096 & -1.235591088 & 4.943509353 \\ \text { H } & 1.950274141 & 0.346168023 & 5.447462390 \\ \text { H } & 0.424963029 & -0.262220017 & 6.073832439\end{array}$

Coord_4

$\begin{array}{lrrr}C & -1.640359120 & -0.676950049 & 0.321810021 \\ C & -2.055079146 & -1.986197143 & 0.747088053 \\ C & -2.669629190 & 0.167484012 & -0.203771014 \\ C & -3.393964246 & -2.406126173 & 0.620330046 \\ C & -4.004730287 & -0.268126021 & -0.325661024 \\ C & -4.374812317 & -1.563304113 & 0.079835008 \\ C & -0.014509003 & 1.233777089 & 0.389940031 \\ C & -0.609948044 & 2.080615149 & 1.318279097\end{array}$




$\begin{array}{lrrr}\text { C } & 0.897445064 & 1.783338130 & -0.550758039 \\ \mathrm{C} & -0.324897024 & 3.465362248 & 1.315154094 \\ \mathrm{C} & 1.192080087 & 3.157334225 & -0.532682038 \\ \mathrm{C} & 0.592001045 & 3.991172289 & 0.376782028 \\ \mathrm{C} & -0.304227022 & -0.230823015 & 0.384136025 \\ \mathrm{H} & -1.345019096 & -2.647787193 & 1.197288085 \\ \mathrm{H} & -2.411317174 & 1.143716080 & -0.560772041 \\ \mathrm{H} & -3.657554265 & -3.391878245 & 0.953821068 \\ \mathrm{H} & -4.733628343 & 0.399407028 & -0.743581053 \\ \mathrm{H} & -5.386495387 & -1.901753139 & -0.032683003 \\ \mathrm{H} & -1.259877090 & 1.676972121 & 2.072151150 \\ \mathrm{H} & 1.345344095 & 1.149759083 & -1.294053093 \\ \mathrm{H} & -0.792954057 & 4.110284294 & 2.034559144 \\ \mathrm{H} & 1.895287138 & 3.551176255 & -1.243937092 \\ \mathrm{H} & 0.822859060 & 5.041119362 & 0.385329029 \\ \mathrm{C} & 0.805521059 & -1.133713080 & 0.369436026 \\ \mathrm{C} & 2.095820153 & -0.781278058 & 0.993345073 \\ \mathrm{O} & 2.219011161 & -0.016487999 & 1.905018136 \\ \mathrm{O} & 3.111896225 & -1.415444099 & 0.443811031 \\ \mathrm{C} & 4.398125316 & -1.169394083 & 0.983360070 \\ \mathrm{H} & 4.659217333 & -0.126758007 & 0.874064065 \\ \mathrm{H} & 5.074355366 & -1.788747131 & 0.416012028 \\ \mathrm{H} & 4.428307321 & -1.439278105 & 2.028994147 \\ \mathrm{C} & 0.735800052 & -2.392484174 & -0.301722024 \\ \mathrm{~N} & 0.664827048 & -3.402216247 & -0.824668057 \\ \mathrm{C} & 1.065461079 & -0.212903015 & 5.211120374 \\ \mathrm{O} & 0.335749026 & 0.387299029 & 4.184352302 \\ \mathrm{H} & 0.885687063 & 0.435846030 & 3.410846244 \\ \mathrm{H} & 1.366211099 & -1.228293088 & 4.960278356 \\ \mathrm{H} & 1.958021143 & 0.354801026 & 5.465418395 \\ \mathrm{H} & 0.431486033 & -0.255724017 & 6.086679439 \\ & & & \\ \mathrm{C} 00 \mathrm{rd} 55 & -1.651486120 & -0.681707051 & 0.313656024 \\ \mathrm{C} & -2.081631149 & -1.969676140 & 0.770127055 \\ \mathrm{C} & -2.05590 \\ \mathrm{C} & -2.65591191 & 0.156368009 & -0.257717020 \\ \mathrm{C} & -3.415472248 & -2.381396169 & 0.632851047 \\ \mathrm{C} & -3.989027285 & -0.271407020 & -0.393305026 \\ \mathrm{C} & -4.376620314 & -1.547655114 & 0.046514003 \\ \mathrm{C} & -0.016266999 & 1.226219089 & 0.412524030 \\ \mathrm{C} & -0.609802044 & 2.066562149 & 1.343264095 \\ \mathrm{C} & 0.893275062 & 1.782066130 & -0.536981036 \\ \mathrm{C} & -0.327756026 & 3.457747250 & 1.344158098 \\ \mathrm{C} & 1.189199087 & 3.165759230 & -0.510091037 \\ \mathrm{C} & 0.593404042 & 3.989734287 & 0.400390027 \\ \mathrm{C} & -0.304434020 & -0.239927017 & 0.400050031 \\ \mathrm{H} & -1.384635098 & -2.622269186 & 1.253761093\end{array}$




\begin{tabular}{|c|c|c|c|}
\hline $\mathrm{H}$ & -2.381170173 & 121670080 & -0.632860048 \\
\hline $\mathrm{H}$ & -3.695830264 & -3.353113239 & 0.993470070 \\
\hline ト & -4.705228337 & 0.387286027 & -0.846259061 \\
\hline ト & -5.389721389 & -1.880700134 & -0.072098003 \\
\hline & -1.259338092 & 1.660243119 & 2.095895153 \\
\hline & 1.331707097 & 1.154430083 & -1.29043009 \\
\hline ト & -0.797395056 & 4.100284294 & 2.0640241 \\
\hline & 1.891138134 & 3.562187254 & -1.221150089 \\
\hline ト & 0.822394061 & 5.040021362 & 0.41526703 \\
\hline 0 & 0.798118060 & -1.140445082 & 0.399283031 \\
\hline c & 2.101588153 & -0.771258055 & 0.99 \\
\hline C & 2.234551160 & -0.010658000 & 1.907122139 \\
\hline $\mathrm{C}$ & 3.110929222 & -1.389973100 & 0.41 \\
\hline C & 4.406351318 & -1.138104081 & 0.93 \\
\hline 卜 & 4.655440335 & -0.091954009 & 0.83 \\
\hline 7 & 5.076035366 & -1.744915125 & 0.34 \\
\hline $\mathrm{H}$ & 4.459658321 & -1.419791100 & 1.97 \\
\hline C & 0.722147051 & -2.424606174 & -0.2 \\
\hline $\mathrm{N}$ & 0.653793046 & -3.451792249 & -0.71 \\
\hline 0 & 1.076184078 & -0.210354016 & 5.2 \\
\hline 0 & 0.349755025 & 0.389731027 & 4.19515030 \\
\hline ト & 0.902165065 & 034 & 3.42 \\
\hline $\mathrm{H}$ & 1.377710100 & -1.225781089 & 4.97 \\
\hline 卜 & 1.967794142 & 11028 & 5.48 \\
\hline $\mathrm{H}$ & 06030 & 017 & $6.0 s$ \\
\hline \multicolumn{4}{|c|}{ Coord_6 } \\
\hline C & -1.661925120 & -0.688376049 & 0.303359023 \\
\hline C & -2.107587150 & -1.953778142 & 0.7 \\
\hline C & -2.641115188 & 0.141059012 & -0.313312022 \\
\hline C & -3.436108245 & -2.357966169 & 0.64375004 \\
\hline $\mathrm{C}$ & -3.971696286 & -0.279711018 & -0.462149034 \\
\hline C & -4.377006317 & -1.535530113 & 0.011927999 \\
\hline C & -0.018409002 & 1.217594087 & 0.43 \\
\hline C & -0.610294046 & 2.051731147 & 1.365283097 \\
\hline C & 0.888510065 & 1.779544130 & -0.525920036 \\
\hline C & -0.331653025 & 3.449414248 & 1.369582101 \\
\hline$C$ & 1.185407087 & 3.172960226 & -0.4 \\
\hline C & 0.593654041 & 3.987395287 & 0.420214028 \\
\hline C & -0.304584021 & -0.250167020 & 0.41408502 \\
\hline $\mathrm{H}$ & -1.423992101 & -2.595786188 & 1.307876092 \\
\hline 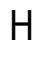 & -2.349928168 & 1.094675081 & -0.70670705 \\
\hline $\mathrm{H}$ & -3.733396266 & -3.314369241 & 1.031395076 \\
\hline 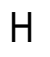 & -4.674826336 & 0.368499028 & -0.949853069 \\
\hline $\mathrm{H}$ & -5.391383387 & -1.863484135 & -0.11255101 \\
\hline $\mathrm{H}$ & -1.259356089 & 1.642934118 & 2.11683815 \\
\hline & 1.317584097 & 1.157678083 & -1.28902209 \\
\hline
\end{tabular}




$\begin{array}{rrrr}\mathrm{H} & -0.803070058 & 4.089781295 & 2.089580153 \\ \mathrm{H} & 1.886015137 & 3.571787260 & -1.201782086 \\ \mathrm{H} & 0.820518059 & 5.038046361 & 0.440934032 \\ \mathrm{C} & 0.790188058 & -1.148696082 & 0.427700029 \\ \mathrm{C} & 2.106802152 & -0.765271055 & 0.993787074 \\ \mathrm{O} & 2.251107164 & -0.009540002 & 1.907347135 \\ \mathrm{O} & 3.107278222 & -1.369966099 & 0.390178028 \\ \mathrm{C} & 4.412090319 & -1.114758083 & 0.882179062 \\ \mathrm{H} & 4.651304334 & -0.065700007 & 0.786727054 \\ \mathrm{H} & 5.073239363 & -1.709835124 & 0.272942021 \\ \mathrm{H} & 4.488435324 & -1.408698100 & 1.918808140 \\ \mathrm{C} & 0.707651050 & -2.456728175 & -0.150144011 \\ \mathrm{~N} & 0.642847046 & -3.500165251 & -0.599987044 \\ \mathrm{C} & 1.087886076 & -0.212509016 & 5.235874375 \\ \mathrm{O} & 0.365614028 & 0.389091029 & 4.204472303 \\ \mathrm{H} & 0.920483065 & 0.440959031 & 3.435074248 \\ \mathrm{H} & 1.388512100 & -1.228348091 & 4.986801360 \\ \mathrm{H} & 1.979485144 & 0.353957026 & 5.496122397 \\ \mathrm{H} & 0.447938032 & -0.254453017 & 6.106983441\end{array}$

\section{Coord_7}

$\begin{array}{lrrr}\text { C } & -1.671648122 & -0.697148051 & 0.291037020 \\ \text { C } & -2.132909154 & -1.938628141 & 0.809916056 \\ \text { C } & -2.625028189 & 0.121309011 & -0.370384026 \\ \text { C } & -3.455855251 & -2.335958169 & 0.653167045 \\ \text { C } & -3.952791286 & -0.293301020 & -0.531971040 \\ \text { C } & -4.376000314 & -1.527127111 & -0.023722000 \\ \text { C } & -0.020962002 & 1.207815087 & 0.450234031 \\ \text { C } & -0.611456045 & 2.036070146 & 1.384326101 \\ \text { C } & 0.883093062 & 1.775648126 & -0.517606037 \\ \text { C } & -0.336665026 & 3.440318247 & 1.391337102 \\ \text { C } & 1.180602084 & 3.178835231 & -0.474822035 \\ \text { C } & 0.592647043 & 3.984086288 & 0.436123033 \\ \text { C } & -0.304657021 & -0.261645018 & 0.426322030 \\ \text { H } & -1.463024103 & -2.568407187 & 1.359686100 \\ \text { H } & -2.317624167 & 1.062444075 & -0.782164054 \\ \text { H } & -3.770212270 & -3.275724236 & 1.067719078 \\ \text { H } & -4.642509334 & 0.342728027 & -1.054098077 \\ \text { H } & -5.391522386 & -1.850310136 & -0.153820010 \\ \text { H } & -1.259942089 & 1.625006117 & 2.135000156 \\ \text { H } & 1.302926093 & 1.159331085 & -1.289844091 \\ \text { H } & -0.810062056 & 4.078749293 & 2.111115153 \\ \text { H } & 1.879798136 & 3.579857260 & -1.185981086 \\ \text { H } & 0.817098060 & 5.035138363 & 0.462147034 \\ \text { C } & 0.781722059 & -1.158567085 & 0.454731033 \\ \text { C } & 2.111509152 & -0.763576057 & 0.991079072 \\ \text { O } & 2.268726162 & -0.013478002 & 1.905812135\end{array}$




$\begin{array}{lrrr}\mathrm{O} & 3.101003222 & -1.355697099 & 0.359854023 \\ \mathrm{C} & 4.415394317 & -1.099778080 & 0.827249061 \\ \mathrm{H} & 4.647006335 & -0.048398001 & 0.739846051 \\ \mathrm{H} & 5.066029365 & -1.683923119 & 0.196637016 \\ \mathrm{H} & 4.514533325 & -1.406544100 & 1.858152135 \\ \mathrm{C} & 0.692199048 & -2.488850182 & -0.074356008 \\ \mathrm{~N} & 0.631984048 & -3.547293253 & -0.486689036 \\ \mathrm{C} & 1.100399080 & -0.219678018 & 5.245546375 \\ \mathrm{O} & 0.383269027 & 0.385133027 & 4.212337301 \\ \mathrm{H} & 0.940635066 & 0.437789031 & 3.444981246 \\ \mathrm{H} & 1.398377101 & -1.236322088 & 4.996624361 \\ \mathrm{H} & 1.992949143 & 0.343747027 & 5.509060394 \\ \mathrm{H} & 0.457465032 & -0.260163020 & 6.114453439\end{array}$

\section{Coord_8}

$\begin{array}{lrrr}C & -1.680637118 & -0.708221053 & 0.276827018 \\ \text { C } & -2.157558156 & -1.924358141 & 0.826883061 \\ \text { C } & -2.607773190 & 0.096848009 & -0.428737031 \\ \text { C } & -3.474697252 & -2.315507167 & 0.661266050 \\ \text { C } & -3.932381284 & -0.312461020 & -0.602520041 \\ \text { C } & -4.373639316 & -1.522665110 & -0.060206004 \\ \text { C } & -0.023962003 & 1.196796088 & 0.465390033 \\ \text { C } & -0.613324046 & 2.019528145 & 1.400385101 \\ \text { C } & 0.876951061 & 1.770247126 & -0.512079035 \\ \text { C } & -0.342869026 & 3.430418245 & 1.409327101 \\ \text { C } & 1.174669086 & 3.183275230 & -0.462395033 \\ \text { C } & 0.590270043 & 3.979732287 & 0.447964030 \\ \text { C } & -0.304644024 & -0.274464019 & 0.436849033 \\ H & -1.501656106 & -2.540199182 & 1.409259102 \\ H & -2.284310162 & 1.024672075 & -0.859058064 \\ H & -3.806231276 & -3.237261231 & 1.102589080 \\ H & -4.608387334 & 0.309622021 & -1.158691082 \\ H & -5.390185387 & -1.841405130 & -0.195646015 \\ H & -1.261107089 & 1.606431117 & 2.150411157 \\ H & 1.287661092 & 1.159202082 & -1.292919092 \\ H & -0.818451061 & 4.067170292 & 2.128512152 \\ H & 1.872347135 & 3.586268258 & -1.173918083 \\ H & 0.811995056 & 5.031237363 & 0.478695036 \\ \text { C } & 0.772700057 & -1.170158083 & 0.480424033 \\ \text { C } & 2.115740152 & -0.766452056 & 0.986589072 \\ \text { O } & 2.287440167 & -0.022856001 & 1.902653137 \\ \text { O } & 3.092147224 & -1.347449099 & 0.327481023 \\ \text { C } & 4.416291320 & -1.093614081 & 0.769788053 \\ H & 4.642728334 & -0.040495003 & 0.691202051 \\ H & 5.054439362 & -1.667605118 & 0.117605008 \\ H & 4.537802328 & -1.413914100 & 1.794093128 \\ \text { C } & 0.675667048 & -2.520972183 & 0.001433001\end{array}$




$\begin{array}{lrrr}\mathrm{N} & 0.621200045 & -3.593133259 & -0.372835027 \\ \mathrm{C} & 1.113514082 & -0.232218016 & 5.253476377 \\ \mathrm{O} & 0.402642031 & 0.377571026 & 4.218773303 \\ \mathrm{H} & 0.962601071 & 0.430372030 & 3.453495247 \\ \mathrm{H} & 1.407014099 & -1.250066091 & 5.004202359 \\ \mathrm{H} & 2.007999145 & 0.326425023 & 5.520536397 \\ \mathrm{H} & 0.467533031 & -0.270494020 & 6.120152440\end{array}$

$\begin{array}{lrrr}\text { Coord_9 } & & & \\ \text { C } & -1.688890119 & -0.721803054 & 0.260889019 \\ \text { C } & -2.181502157 & -1.911106140 & 0.842078063 \\ \text { C } & -2.589417185 & 0.067389003 & -0.488143035 \\ \text { C } & -3.492625254 & -2.296759163 & 0.668239050 \\ \text { C } & -3.910561280 & -0.337500026 & -0.673502046 \\ \text { C } & -4.369974314 & -1.522387112 & -0.097251009 \\ \text { C } & -0.027459002 & 1.184451086 & 0.478102035 \\ \mathrm{C} & -0.615941045 & 2.002061142 & 1.413454102 \\ \mathrm{C} & 0.869991062 & 1.763200125 & -0.509385036 \\ \mathrm{C} & -0.350349026 & 3.419675249 & 1.423446101 \\ \mathrm{C} & 1.167473085 & 3.186161230 & -0.453639033 \\ \mathrm{C} & 0.586398042 & 3.974256287 & 0.455569032 \\ \mathrm{C} & -0.304546021 & -0.288723023 & 0.445765034 \\ \mathrm{H} & -1.539815109 & -2.511232183 & 1.456679104 \\ \mathrm{H} & -2.250074162 & 0.981027070 & -0.937185068 \\ \mathrm{H} & -3.841404274 & -3.199075229 & 1.136178082 \\ \mathrm{H} & -4.572601331 & 0.268801018 & -1.263279091 \\ \mathrm{H} & -5.387433385 & -1.837027130 & -0.237728016 \\ \mathrm{H} & -1.262864090 & 1.587193116 & 2.163106157 \\ \mathrm{H} & 1.271688094 & 1.157090082 & -1.298278092 \\ \mathrm{H} & -0.828317057 & 4.055033294 & 2.141642156 \\ \mathrm{H} & 1.863495132 & 3.590875260 & -1.165793085 \\ \mathrm{H} & 0.805056055 & 5.026278364 & 0.490340036 \\ \mathrm{C} & 0.763080054 & -1.183573086 & 0.504832037 \\ \mathrm{C} & 2.119503152 & -0.774202058 & 0.980462073 \\ \mathrm{O} & 2.307253166 & -0.038110001 & 1.898024138 \\ \mathrm{O} & 3.080724224 & -1.345518099 & 0.293290024 \\ \mathrm{C} & 4.414769316 & -1.096756081 & 0.710082053 \\ \mathrm{H} & 4.638623336 & -0.042495002 & 0.641104048 \\ \mathrm{H} & 5.038463363 & -1.661335119 & 0.036202005 \\ \mathrm{H} & 4.558038330 & -1.431448102 & 1.726881124 \\ \mathrm{C} & 0.657908050 & -2.553094184 & 0.077222005 \\ \mathrm{~N} & 0.610486042 & -3.637645263 & -0.258490021 \\ \mathrm{C} & 1.126973081 & -0.250540016 & 5.259726378 \\ \mathrm{O} & 0.423634032 & 0.366074026 & 4.223825305 \\ \mathrm{H} & 0.986332072 & 0.418328030 & 3.460699249 \\ \mathrm{H} & 1.414064100 & -1.269988091 & 5.009577361 \\ \mathrm{H} & 2.024393146 & 0.301456022 & 5.530679396\end{array}$


$\mathrm{H}$

Coord_10

\begin{tabular}{|c|c|c|c|}
\hline & -1.709936123 & -0.669024048 & 0.194563012 \\
\hline & -2.245596163 & -1.823858131 & 94847057 \\
\hline & -2.562954185 & 0.125829010 & -0.595818043 \\
\hline & -3.557087255 & -2.176119154 & 0.603381044 \\
\hline & -3.887260277 & -0.246572018 & -0.8024010 \\
\hline C & -4.392184317 & -1.398926101 & -0.2041460 \\
\hline & -0.007244003 & 1.202845086 & 0.4636950 \\
\hline & -0.583352040 & 2.020499143 & 1.402012099 \\
\hline & 0.903855067 & 1.775581127 & -0.524747035 \\
\hline & -0.293794023 & 3.439093249 & 1.424739103 \\
\hline & 1.229893087 & 3.201601230 & -0.450956030 \\
\hline & 0.663134046 & 3.986550286 & 0.459496036 \\
\hline & -0.310412025 & -0.266072019 & 0.415335030 \\
\hline & -1.634149119 & -2.424327176 & 1.440008105 \\
\hline & -2.186775159 & 1.020415072 & -1.054726078 \\
\hline & -3.944085283 & -3.053552221 & 1.088776078 \\
\hline & -4.519297328 & 0.360188024 & -1.424128104 \\
\hline & -5.415843388 & -1.687483122 & -0.357314025 \\
\hline & -1.241903088 & 1.611825116 & 771154 \\
\hline & 1.288549094 & 1.172198082 & -1.323704095 \\
\hline & -0.765111052 & 4.077607292 & 2.143889153 \\
\hline & 1.936347138 & 3.598796260 & -1.15703308 \\
\hline & 0.899909066 & 5.0341 & 0.5 \\
\hline & 0.727576050 & -1.182781088 & 0.488858033 \\
\hline & 2.103276149 & -0.803042060 & 0.944398067 \\
\hline & 2.318508168 & -0.087221005 & 1.870472133 \\
\hline & 3.039344220 & -1.379986102 & 0.229789018 \\
\hline & 4.385875317 & -1.170523083 & 0.629914044 \\
\hline & 4.632059332 & -0.120212010 & 0.580915041 \\
\hline & 4.985402358 & -1.733428127 & -0.066780004 \\
\hline & 4.537119324 & -1.531808113 & 1.636336119 \\
\hline & 0.586932040 & -2.563449182 & 0.102287009 \\
\hline & 0.527003040 & -3.655627261 & -0.203302014 \\
\hline & 1.107701081 & -0.304165024 & 5.234401379 \\
\hline & 0.432300029 & 0.343559026 & 4.198798301 \\
\hline & 1.003139070 & 0.388689029 & 3.441463249 \\
\hline & 1.366513101 & -1.329169094 & 4.9762763 \\
\hline & 2.018574144 & 0.218177017 & 5.518867398 \\
\hline & 13032 & -0.32 & 6.0925 \\
\hline
\end{tabular}

LIIC path connecting the $V^{\prime}\left({ }^{1} \pi \pi^{*}\right)$ minimum and $V^{\prime}\left({ }^{1} \pi \pi^{*}\right) / T_{2}\left({ }^{3} \pi \pi^{*}\right)$ crossing point Coord_1
C -1.615763115
$-0.656429047$
0.271572018
$\begin{array}{llll}\text { C } & -2.103145151 & -1.966966143 & 0.642862048\end{array}$ 


$\begin{array}{lrrr}\text { C } & -2.645629192 & 0.238961015 & -0.211146014 \\ \text { C } & -3.461291250 & -2.390037173 & 0.403339031 \\ \text { C } & -3.999261287 & -0.196583014 & -0.452229035 \\ \text { C } & -4.416354318 & -1.516542111 & -0.167266012 \\ \text { C } & 0.020046001 & 1.216707086 & 0.403532028 \\ \text { C } & -0.453628031 & 2.068871150 & 1.409424100 \\ \text { C } & 0.780459055 & 1.755676125 & -0.631062047 \\ \text { C } & -0.167195012 & 3.429511246 & 1.366404101 \\ \text { C } & 1.072955075 & 3.118598223 & -0.657238048 \\ \text { C } & 0.598750045 & 3.952057285 & 0.330097021 \\ \text { C } & -0.287519023 & -0.259383018 & 0.421729031 \\ \text { H } & -1.460380106 & -2.644785191 & 1.160691086 \\ \text { H } & -2.397847173 & 1.257658091 & -0.424549032 \\ \text { H } & -3.732463269 & -3.387610245 & 0.688091050 \\ \text { H } & -4.690968338 & 0.511194039 & -0.866051062 \\ \text { H } & -5.417658392 & -1.840667134 & -0.368797029 \\ \text { H } & -1.010295075 & 1.664902118 & 2.235058160 \\ \text { H } & 1.152946085 & 1.116348080 & -1.411513101 \\ \text { H } & -0.530240038 & 4.076690291 & 2.144123156 \\ \text { H } & 1.675135120 & 3.514600254 & -1.454727107 \\ \text { H } & 0.824341058 & 5.003416361 & 0.303566021 \\ \text { C } & 0.781194056 & -1.162559082 & 0.494153034 \\ \text { C } & 2.113601154 & -0.771361054 & 0.998906070 \\ \text { O } & 2.310019165 & 0.058767007 & 1.837387130 \\ \text { O } & 3.088139220 & -1.464707104 & 0.441251031 \\ \text { C } & 4.405653317 & -1.205425085 & 0.887949063 \\ \text { H } & 4.670853337 & -0.174054012 & 0.705752050 \\ \text { H } & 5.041486362 & -1.864477137 & 0.317931025 \\ \text { H } & 4.496870325 & -1.416983100 & 1.943914140 \\ \mathrm{C} & 0.679138048 & -2.503717178 & 0.005525002 \\ \text { N } & 0.588236044 & -3.570279258 & -0.384584027 \\ \text { C } & 1.116611082 & -0.301590021 & 5.181102371 \\ \text { O } & 0.434719031 & 0.356166023 & 4.157248298 \\ \text { H } & 1.007624074 & 0.424873032 & 3.401409246 \\ \text { H } & 1.388091102 & -1.318638096 & 4.904811351 \\ \text { H } & 2.021349144 & 0.225804017 & 5.475578392 \\ \text { H } & 0.459340034 & -0.349627027 & 6.039230433\end{array}$

Coord_2

$\begin{array}{lrrr}C & -1.619112114 & -0.655995048 & 0.269748018 \\ C & -2.102622149 & -1.964944141 & 0.637731045 \\ C & -2.648284191 & 0.239487017 & -0.208938017 \\ C & -3.462131251 & -2.392585172 & 0.403161027 \\ C & -4.003902288 & -0.195271014 & -0.450350032 \\ C & -4.420881319 & -1.518112111 & -0.166556010 \\ C & 0.019123000 & 1.216918090 & 0.400526031 \\ C & -0.449967035 & 2.069301149 & 1.408113100\end{array}$




\begin{tabular}{|c|c|c|c|}
\hline C & 0.777982056 & 1.755210126 & -0.635338048 \\
\hline C & -0.161139013 & 3.429559248 & 1.365690098 \\
\hline C & 1.073611075 & 3.118005227 & -0.660991047 \\
\hline C & 0.603549042 & 3.951418282 & 0.328022022 \\
\hline C & -0.292324021 & -0.258225019 & 0.420049031 \\
\hline ト & -1.457367103 & -2.643338188 & 1.152155081 \\
\hline $\mathrm{H}$ & -2.400664174 & 1.258443089 & -0.421481032 \\
\hline $\mathrm{H}$ & -3.731352266 & -3.389749243 & 0.690712049 \\
\hline 1 & -4.695824338 & 0.512313037 & -0.863936063 \\
\hline 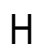 & -5.421749392 & -1.843118135 & -0.368668026 \\
\hline $\mathrm{H}$ & -1.006460075 & 1.665710118 & 64158 \\
\hline 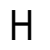 & 1.146487085 & 1.116224078 & -1.418072099 \\
\hline r & -0.521415039 & 4.076965294 & 8153 \\
\hline $\mathrm{H}$ & 1.674506118 & 3.513601252 & -1.459648106 \\
\hline $\mathrm{H}$ & 0.831238058 & 5.002363362 & $0.30^{\prime}$ \\
\hline C & 819055 & -1.164247083 & 0.4 \\
\hline C & 2.113673154 & -0.7725 & 1.0029 \\
\hline$v$ & 2.306190165 & 0.05 & 1.84 \\
\hline 0 & 3.088443222 & -1.460376106 & 0.440770030 \\
\hline 0 & 4.406523316 & -1.202803086 & $0.8 \varepsilon$ \\
\hline $\mathrm{H}$ & 4.671104336 & -0.170633013 & 0.710165049 \\
\hline $\mathrm{H}$ & 5.042014365 & -1.859 & 0.315408024 \\
\hline $\mathrm{H}$ & 4.497417325 & -1.418793103 & 1.943460138 \\
\hline C & 0.683184048 & -2.504658183 & 0.000290000 \\
\hline $\mathrm{N}$ & 0.599426042 & -3.570058258 & -0.393712027 \\
\hline C & 1.115966078 & -0.300505022 & 354375 \\
\hline 0 & 0.431062030 & 0.357411024 & 4.16 \\
\hline $\mathrm{H}$ & 1.002711071 & 0.429149033 & 3.410260244 \\
\hline$\Pi$ & 1.388940098 & -1.316653094 & 212351 \\
\hline $\mathrm{H}$ & 2.020007146 & 5017 & 5.482339393 \\
\hline $\mathrm{H}$ & 0.460016032 & 6025 & 272435 \\
\hline \multicolumn{4}{|c|}{ Coord_3 } \\
\hline C & -1.622 & -0.655562048 & 0.267841017 \\
\hline C & -2.102149150 & -1.962896141 & 0.632554046 \\
\hline C & -2.650987191 & 0.239974019 & -0.206839014 \\
\hline C & -3.462974251 & -2.395155175 & 0.402941027 \\
\hline C & -4.00 & -0.194022013 & 5033 \\
\hline C & -4.425423321 & -1.519746110 & -0.165913012 \\
\hline C & 0.018179001 & 1.217101090 & 0.397420030 \\
\hline C & -0.446320030 & 2.069710150 & 1.406676103 \\
\hline C & 0.775509057 & 1.754696125 & -0.639702045 \\
\hline C & -0.155064010 & 3.429573245 & 1.364838096 \\
\hline C & 1.074302080 & 3.117348222 & -0.664847045 \\
\hline $\mathrm{C}$ & 0.608390045 & 3.950723282 & 0.325819025 \\
\hline C & -0.297169020 & -0.257077017 & 0.418285029 \\
\hline & -1.454405102 & -2.641832192 & 1.14 \\
\hline
\end{tabular}




$\begin{array}{rrrr}\mathrm{H} & -2.403544174 & 1.259186091 & -0.418550030 \\ \mathrm{H} & -3.730208270 & -3.391903244 & 0.693304049 \\ \mathrm{H} & -4.700734336 & 0.513345038 & -0.861933063 \\ \mathrm{H} & -5.425846388 & -1.845656133 & -0.368614029 \\ \mathrm{H} & -1.002660074 & 1.666518119 & 2.232939159 \\ \mathrm{H} & 1.140023084 & 1.116052080 & -1.424695102 \\ \mathrm{H} & -0.512566036 & 4.077215293 & 2.144738155 \\ \mathrm{H} & 1.673930120 & 3.512524255 & -1.464663103 \\ \mathrm{H} & 0.838200062 & 5.001244359 & 0.300130021 \\ \mathrm{C} & 0.780393056 & -1.165952082 & 0.494445034 \\ \mathrm{C} & 2.113679155 & -0.773730053 & 1.006947071 \\ \mathrm{O} & 2.302245165 & 0.046423006 & 1.855088135 \\ \mathrm{O} & 3.088663222 & -1.456042107 & 0.440293029 \\ \mathrm{C} & 4.407318315 & -1.200169085 & 0.888893062 \\ \mathrm{H} & 4.671272334 & -0.167218010 & 0.714516051 \\ \mathrm{H} & 5.042455365 & -1.854397135 & 0.312858025 \\ \mathrm{H} & 4.497912322 & -1.420563101 & 1.942951138 \\ \mathrm{C} & 0.687201048 & -2.505598181 & -0.004945003 \\ \mathrm{~N} & 0.610621046 & -3.569783255 & -0.402821030 \\ \mathrm{C} & 1.115441081 & -0.299821023 & 5.195479376 \\ \mathrm{O} & 0.427461031 & 0.358373026 & 4.175952301 \\ \mathrm{H} & 0.997790072 & 0.433234032 & 3.419093244 \\ \mathrm{H} & 1.389883103 & -1.315027097 & 4.915343355 \\ \mathrm{H} & 2.018802145 & 0.230466016 & 5.488981393 \\ \mathrm{H} & 0.460876035 & -0.353169028 & 6.055219433\end{array}$

\section{Coord_4}

$\begin{array}{rrrr}\text { C } & -1.625937119 & -0.655129049 & 0.265852019 \\ \text { C } & -2.101726152 & -1.960823142 & 0.627332046 \\ \text { C } & -2.653740193 & 0.240424015 & -0.204850017 \\ \text { C } & -3.463818252 & -2.397749175 & 0.402681031 \\ \text { C } & -4.013310287 & -0.192838016 & -0.446872031 \\ \text { C } & -4.429983321 & -1.521445108 & -0.165338013 \\ \text { C } & 0.017215999 & 1.217258086 & 0.394214026 \\ \text { C } & -0.442685032 & 2.070098148 & 1.405113099 \\ \text { C } & 0.773040054 & 1.754134128 & -0.644155045 \\ \text { C } & -0.148970010 & 3.429554247 & 1.363850096 \\ \text { C } & 1.075030080 & 3.116629223 & -0.668806048 \\ \text { C } & 0.613274044 & 3.949974285 & 0.323490021 \\ \text { C } & -0.302054019 & -0.255940016 & 0.416437031 \\ \text { H } & -1.451496104 & -2.640268192 & 1.134982084 \\ \text { H } & -2.406489172 & 1.259888092 & -0.415757028 \\ \text { H } & -3.729031268 & -3.394073246 & 0.695868051 \\ \text { H } & -4.705698336 & 0.514289038 & -0.860042064 \\ \text { H } & -5.429950391 & -1.848281132 & -0.368634026 \\ \text { H } & -0.998895073 & 1.667328120 & 2.231684162 \\ \text { H } & 1.133556083 & 1.115833080 & -1.431384103\end{array}$




$\begin{array}{rrrr}\mathrm{H} & -0.503693036 & 4.077440294 & 2.144815155 \\ \mathrm{H} & 1.673408118 & 3.511369251 & -1.469770108 \\ \mathrm{H} & 0.845229059 & 5.000060362 & 0.298207022 \\ \mathrm{C} & 0.779917056 & -1.167674083 & 0.494523034 \\ \mathrm{C} & 2.113618151 & -0.774936054 & 1.010916070 \\ \mathrm{O} & 2.298185163 & 0.040197002 & 1.863816136 \\ \mathrm{O} & 3.088802221 & -1.451704102 & 0.439820029 \\ \mathrm{C} & 4.408041319 & -1.197519087 & 0.889310064 \\ \mathrm{H} & 4.671359336 & -0.163805013 & 0.718806053 \\ \mathrm{H} & 5.042813364 & -1.849331131 & 0.310281021 \\ \mathrm{H} & 4.498359324 & -1.422292103 & 1.942390141 \\ \mathrm{C} & 0.691189050 & -2.506538180 & -0.010179000 \\ \mathrm{~N} & 0.621822046 & -3.569453254 & -0.411909031 \\ \mathrm{C} & 1.115038081 & -0.299534019 & 5.202479375 \\ \mathrm{O} & 0.423918031 & 0.359054024 & 4.185316299 \\ \mathrm{H} & 0.992862072 & 0.437130031 & 3.427910247 \\ \mathrm{H} & 1.390921100 & -1.313760093 & 4.920204356 \\ \mathrm{H} & 2.017736144 & 0.232141015 & 5.495506398 \\ \mathrm{H} & 0.461922033 & -0.355692023 & 6.063073435\end{array}$

Coord_5

$\begin{array}{lrrr}\text { C } & -1.629414115 & -0.654695050 & 0.263781021 \\ \text { C } & -2.101352151 & -1.958724139 & 0.622065044 \\ \text { C } & -2.656542192 & 0.240837017 & -0.202971014 \\ \text { C } & -3.464663247 & -2.400364173 & 0.402379030 \\ \text { C } & -4.018078291 & -0.191717012 & -0.445273032 \\ \text { C } & -4.434560318 & -1.523208109 & -0.164831014 \\ \text { C } & 0.016232999 & 1.217387089 & 0.390908028 \\ \text { C } & -0.439062031 & 2.070465149 & 1.403424103 \\ \text { C } & 0.770576056 & 1.753526125 & -0.648696048 \\ \text { C } & -0.142855013 & 3.429502245 & 1.362725096 \\ \text { C } & 1.075796080 & 3.115848226 & -0.672867049 \\ \text { C } & 0.618201045 & 3.949171285 & 0.321035025 \\ \text { C } & -0.306979024 & -0.254813017 & 0.414505031 \\ \text { H } & -1.448640103 & -2.638647190 & 1.126345080 \\ \text { H } & -2.409499175 & 1.260552093 & -0.413101029 \\ H & -3.727822268 & -3.396259245 & 0.698403048 \\ \text { H } & -4.710719339 & 0.515146035 & -0.858264060 \\ \text { H } & -5.434060389 & -1.850993134 & -0.368730024 \\ \text { H } & -0.995163072 & 1.668139121 & 2.230300163 \\ \text { H } & 1.127085082 & 1.115566078 & -1.438139104 \\ \text { H } & -0.494795037 & 4.077640291 & 2.144741155 \\ H & 1.672943119 & 3.510137253 & -1.474970105 \\ \text { H } & 0.852326062 & 4.998812361 & 0.296149020 \\ \text { C } & 0.779391054 & -1.169413086 & 0.494554034 \\ \text { C } & 2.113493154 & -0.776157056 & 1.014850074 \\ \text { O } & 2.294012167 & 0.033934004 & 1.872460135\end{array}$




$\begin{array}{lrrr}\mathrm{O} & 3.088859224 & -1.447363103 & 0.439351030 \\ \mathrm{C} & 4.408690318 & -1.194853086 & 0.889690066 \\ \mathrm{H} & 4.671364337 & -0.160393010 & 0.723036053 \\ \mathrm{H} & 5.043088362 & -1.844243134 & 0.307679020 \\ \mathrm{H} & 4.498756323 & -1.423977104 & 1.941778137 \\ \mathrm{C} & 0.695149052 & -2.507478179 & -0.015414002 \\ \mathrm{~N} & 0.633027046 & -3.569068257 & -0.420978028 \\ \mathrm{C} & 1.114756082 & -0.299642024 & 5.209354377 \\ \mathrm{O} & 0.420433028 & 0.359454024 & 4.194690303 \\ \mathrm{H} & 0.987928071 & 0.440838034 & 3.436713248 \\ \mathrm{H} & 1.392052100 & -1.312848093 & 4.924797355 \\ \mathrm{H} & 2.016810148 & 0.233380015 & 5.501914395 \\ \mathrm{H} & 0.463156032 & -0.358712027 & 6.070831438\end{array}$

\section{Coord_6}

$\begin{array}{rrrr}\text { C } & -1.632933118 & -0.654260045 & 0.261628021 \\ \text { C } & -2.101028151 & -1.956599143 & 0.616753046 \\ \text { C } & -2.659393193 & 0.241215019 & -0.201202017 \\ \text { C } & -3.465510248 & -2.403000174 & 0.402036028 \\ \text { C } & -4.022889290 & -0.190658012 & -0.443767030 \\ \text { C } & -4.439153322 & -1.525036110 & -0.164392013 \\ \text { C } & 0.015231002 & 1.217490088 & 0.387502026 \\ \text { C } & -0.435450031 & 2.070810151 & 1.401611099 \\ \text { C } & 0.768118055 & 1.752871125 & -0.653326047 \\ \text { C } & -0.136719012 & 3.429417248 & 1.361465099 \\ \text { C } & 1.076601080 & 3.115005226 & -0.677030050 \\ \text { C } & 0.623173046 & 3.948312282 & 0.318455021 \\ \text { C } & -0.311944024 & -0.253696019 & 0.412489030 \\ \text { H } & -1.445835103 & -2.636966190 & 1.117675082 \\ \text { H } & -2.412574176 & 1.261177088 & -0.410583029 \\ \text { H } & -3.726579267 & -3.398459247 & 0.700908051 \\ \text { H } & -4.715795340 & 0.515916035 & -0.856598062 \\ \text { H } & -5.438177393 & -1.853791132 & -0.368901028 \\ \text { H } & -0.991464070 & 1.668952123 & 2.228787160 \\ \text { H } & 1.120612080 & 1.115253080 & -1.444959103 \\ \text { H } & -0.485870034 & 4.077814295 & 2.144516154 \\ \text { H } & 1.672534118 & 3.508827253 & -1.480263105 \\ \text { H } & 0.859493064 & 4.997498361 & 0.293956020 \\ \text { C } & 0.778814055 & -1.171169087 & 0.494539037 \\ \text { C } & 2.113301153 & -0.777393055 & 1.018749073 \\ \text { O } & 2.289728165 & 0.027635001 & 1.881020133 \\ \text { O } & 3.088835220 & -1.443018102 & 0.438886032 \\ \text { C } & 4.409267317 & -1.192168083 & 0.890034063 \\ \text { H } & 4.671286336 & -0.156979013 & 0.727206054 \\ \text { H } & 5.043282363 & -1.839133134 & 0.305051020 \\ \text { H } & 4.499103326 & -1.425615103 & 1.941115142 \\ \text { C } & 0.699079051 & -2.508418183 & -0.020648999\end{array}$




\begin{tabular}{|c|c|c|c|}
\hline $\mathrm{N}$ & 0.644235046 & -3.568628257 & -0.430027033 \\
\hline C & 1.114597081 & -0.300144023 & 5.216104377 \\
\hline C & 0.417009029 & 0.359576025 & 4.204075304 \\
\hline & 0.982990069 & 0.444359031 & 3.445505248 \\
\hline ト & 1.393278103 & -1.312289096 & 4.929121352 \\
\hline & 2.016025145 & 0.234185016 & 5.50820 \\
\hline & 0.464578032 & -0.362228024 & 6.07 \\
\hline \multicolumn{4}{|c|}{ Coord_7 } \\
\hline C & -1.6364 & -0.653823045 & 3019 \\
\hline C & -2.100753154 & -1.954447138 & 0.6 \\
\hline C & -2.662294190 & 0.241559016 & -0.19 \\
\hline $\mathrm{C}$ & -3.466356249 & -2.405658173 & 0.4 \\
\hline 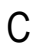 & -4.027743290 & -0.189662016 & -0.4 \\
\hline C & -4.443762318 & -1.526927109 & -0.1 \\
\hline C & 0.014212002 & 1.217567089 & \\
\hline C & -0.431849033 & 2.071135151 & 1.3 \\
\hline C & 0.765665053 & 1.752169124 & -0.6 \\
\hline C & -0.130561008 & 3.429298247 & 1.36 \\
\hline C & 1.077444075 & 3.114100222 & -0.6 \\
\hline C & 0.628191043 & 3.947399282 & 0.31 \\
\hline$C$ & -0.316948025 & -0.252586017 & 0.4 \\
\hline $\mathrm{H}$ & -1.443081106 & -2.635226192 & \\
\hline $\mathrm{H}$ & -2.415715176 & 1.261766089 & -0.40 \\
\hline $\mathrm{H}$ & -3.725301267 & -3.400672245 & \\
\hline $\mathrm{H}$ & -4.720926337 & 0.516599039 & -0.855045059 \\
\hline $\mathrm{H}$ & -5.442298393 & -1.856676133 & -0.3 \\
\hline $\mathrm{H}$ & -0.987798073 & 1.669766119 & 2.22 \\
\hline $\mathrm{H}$ & 1.114138078 & 1.114894081 & -1.4 \\
\hline $\mathrm{H}$ & -0.476918032 & 4.077962295 & 2.14 \\
\hline $\mathrm{H}$ & 1.672182120 & 3.507440253 & -1.48 \\
\hline $\mathrm{H}$ & 0.866730060 & 4.996118362 & 0.29 \\
\hline C & 0.778186054 & -1.172941084 & 0.494477033 \\
\hline C & 2.113043152 & -0.778643057 & 1.02 \\
\hline 0 & 2.285334163 & 0.021300003 & 1.88949613 \\
\hline 0 & 3.088731221 & -1.438667101 & 0.43 \\
\hline C & 4.409770316 & -1.189463088 & 0.890344066 \\
\hline $\mathrm{H}$ & 4.671125334 & -0.153560009 & 0.73131705 \\
\hline $\mathrm{H}$ & 5.043395364 & -1.833997130 & 0.302401022 \\
\hline $\mathrm{H}$ & 4.499401321 & -1.427205101 & 1.94040313 \\
\hline C & 0.702980050 & -2.509358181 & -0.02588300 \\
\hline $\mathrm{N}$ & 0.655448048 & -3.568132259 & -0.439054030 \\
\hline C & 1.114563081 & -0.301038020 & 5.22273037 \\
\hline 0 & 0.413647029 & 0.359421024 & 4.213471301 \\
\hline $\mathrm{H}$ & 0.978050072 & 0.447693033 & 3.45 \\
\hline $\mathrm{H}$ & 1.394597098 & -1.312080093 & 4.93317835 \\
\hline 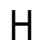 & 2.015384147 & 0.234558017 & 5.51 \\
\hline
\end{tabular}


Coord_8

$\begin{array}{lrrr}\mathrm{C} & -1.640100120 & -0.653382045 & 0.257075017 \\ \mathrm{C} & -2.100527153 & -1.952268140 & 0.605992042 \\ \mathrm{C} & -2.665244194 & 0.241868018 & -0.197994012 \\ \mathrm{C} & -3.467203250 & -2.408335175 & 0.401225027 \\ \mathrm{C} & -4.032640291 & -0.188728013 & -0.441037031 \\ \mathrm{C} & -4.448386322 & -1.528881112 & -0.163720011 \\ \mathrm{C} & 0.013174000 & 1.217618085 & 0.380392027 \\ \mathrm{C} & -0.428258030 & 2.071439147 & 1.397612101 \\ \mathrm{C} & 0.763219053 & 1.751421127 & -0.662850047 \\ \mathrm{C} & -0.124380011 & 3.429145246 & 1.358544099 \\ \mathrm{C} & 1.078328076 & 3.113132224 & -0.685660047 \\ \mathrm{C} & 0.633256047 & 3.946430284 & 0.312923024 \\ \mathrm{C} & -0.321991021 & -0.251485016 & 0.408205028 \\ \mathrm{H} & -1.440379106 & -2.633428190 & 1.100234078 \\ \mathrm{H} & -2.418922176 & 1.262319090 & -0.405959030 \\ \mathrm{H} & -3.723989268 & -3.402898245 & 0.705830051 \\ \mathrm{H} & -4.726114338 & 0.517197036 & -0.853605062 \\ \mathrm{H} & -5.446425393 & -1.859647135 & -0.369471026 \\ \mathrm{H} & -0.984164070 & 1.670582121 & 2.225376158 \\ \mathrm{H} & 1.107662080 & 1.114490081 & -1.458796104 \\ \mathrm{H} & -0.467937032 & 4.078083292 & 2.143619157 \\ \mathrm{H} & 1.671889120 & 3.505976253 & -1.491122108 \\ \mathrm{H} & 0.874038062 & 4.994672358 & 0.289173019 \\ \mathrm{C} & 0.777508056 & -1.174729084 & 0.494368033 \\ \mathrm{C} & 2.112719153 & -0.779907055 & 1.026441072 \\ \mathrm{O} & 2.280831166 & 0.014929000 & 1.897888139 \\ \mathrm{O} & 3.088547221 & -1.434312105 & 0.437968031 \\ \mathrm{C} & 4.410202315 & -1.186735084 & 0.890621064 \\ \mathrm{H} & 4.670881336 & -0.150134010 & 0.735370053 \\ \mathrm{H} & 5.043428363 & -1.828833133 & 0.299728021 \\ \mathrm{H} & 4.499648325 & -1.428745102 & 1.939642140 \\ \mathrm{C} & 0.706850050 & -2.510299180 & -0.031118004 \\ \mathrm{~N} & 0.666663049 & -3.567580258 & -0.448059033 \\ \mathrm{C} & 1.114654078 & -0.302320021 & 5.229229375 \\ \mathrm{O} & 0.410351027 & 0.358991025 & 4.222879305 \\ \mathrm{H} & 0.973108070 & 0.450843035 & 3.463062248 \\ \mathrm{H} & 1.396008102 & -1.312218097 & 4.936966357 \\ \mathrm{H} & 2.014889144 & 0.234502019 & 5.520435398 \\ \mathrm{H} & 0.467995035 & -0.370732029 & 6.093522436 \\ & & & \end{array}$

Coord_9

$\begin{array}{lrrr}C & -1.643747119 & -0.652938049 & 0.254674018 \\ \text { C } & -2.100350153 & -1.950061142 & 0.600542045 \\ \text { C } & -2.668245190 & 0.242145016 & -0.196555015\end{array}$




\begin{tabular}{|c|c|c|c|}
\hline & 51 & -2.4 & 0.40075702 \\
\hline$C$ & -4.037579293 & -0.187855013 & -0 \\
\hline$C$ & -4.453026323 & -1.530899108 & -0.1634870 \\
\hline & 0.012118000 & 1.217643089 & 0.3766890 \\
\hline U & -0.424677030 & 2.071722151 & 395428102 \\
\hline & 0.760781054 & 1.750627128 & \\
\hline 0 & -0.118175006 & 3.428958245 & 3568850 \\
\hline & 1.079253078 & 3.112103223 & -0.69012504 \\
\hline & 0.638368048 & 3.945406284 & 0.309973024 \\
\hline 乙 & -0.327074022 & -0.250392016 & 0.405935 \\
\hline & -1.437728103 & -2.631569190 & \\
\hline & -2.422196174 & 1.262837091 & -0.403853 \\
\hline & -3.722642268 & -3.405137247 & 0.7082440 \\
\hline & 58342 & 9036 & -0.85 \\
\hline & -5.450555394 & -1.86 & -0.3 \\
\hline & -0.980561071 & 1.671399118 & 2.223479 \\
\hline & 1.101185078 & 41079 & -1.465813106 \\
\hline & -0.458927032 & 78295 & \\
\hline & 1.671 & 3.5 & -1.49 \\
\hline$\theta$ & 0.8814 & 4.993159361 & 0.2865840 \\
\hline 乙 & 0.776778056 & -1.176533087 & 0.4942130 \\
\hline & 2.11 & 36055 & \\
\hline & 2.27 & 03 & 1.9 \\
\hline 0 & 34220 & -1.4 & 0.4 \\
\hline $\mathrm{C}$ & 4.410560320 & 81087 & 0.8908640 \\
\hline П & & & \\
\hline & & & \\
\hline & 4.499 & -1.4 & 1.9 \\
\hline$C$ & 0.710689051 & -2.51 & -0.0363 \\
\hline IN & 0.67788 & -3.5 & -0.4 \\
\hline 乙 & & -0.3 & 79 \\
\hline 0 & & & 4.2 \\
\hline $\mathrm{H}$ & 0.968 & 0.453808031 & 3.47183225 \\
\hline$H$ & 1.397513103 & -1.31 & 4.940487 \\
\hline $\mathrm{H}$ & & & 5.52 \\
\hline & 0.46999 & -0.3757 & \\
\hline \multicolumn{4}{|c|}{ Coord_10 } \\
\hline C & -1.64 & & 0.252 \\
\hline C & -2.0 & 012141 & 389042 \\
\hline C & -2.66 & 736019 & -0.1980620 \\
\hline$C$ & -3.46060 & -2.423945174 & 0.3960590 \\
\hline C & -4.036828289 & -0.198727012 & -0.4450670 \\
\hline$c$ & -4.4 & -1.545476110 & -0.170421014 \\
\hline C & 0.011 & & \\
\hline C & $-0.42517 \varsigma$ & 2.068644149 & \\
\hline & 0.7605 & 1.74839 & -0.66 \\
\hline
\end{tabular}




$\begin{array}{rrrr}\text { C } & -0.118847008 & 3.426050247 & 1.358163096 \\ \text { C } & 1.079492076 & 3.110302221 & -0.688322052 \\ \text { C } & 0.638320046 & 3.942963284 & 0.311847021 \\ \text { C } & -0.328609023 & -0.252735017 & 0.407157029 \\ \text { H } & -1.428217105 & -2.635256191 & 1.083984076 \\ \text { H } & -2.423041175 & 1.255134091 & -0.404364027 \\ \text { H } & -3.711658268 & -3.418033243 & 0.706058052 \\ \text { H } & -4.731336339 & 0.504831037 & -0.859517062 \\ \text { H } & -5.445507392 & -1.880557134 & -0.380016026 \\ \text { H } & -0.982418071 & 1.667920121 & 2.222726160 \\ \text { H } & 1.100377077 & 1.112649079 & -1.465892106 \\ \text { H } & -0.460298035 & 4.075056292 & 2.144100152 \\ \text { H } & 1.672070120 & 3.503146250 & -1.494503106 \\ \text { H } & 0.881465064 & 4.990737359 & 0.289158023 \\ \text { C } & 0.781340056 & -1.179360084 & 0.500833034 \\ \text { C } & 2.114904152 & -0.780446059 & 1.044271075 \\ \text { O } & 2.270483163 & 0.004710000 & 1.924887138 \\ \text { O } & 3.093957222 & -1.421618100 & 0.450160031 \\ \text { C } & 4.415108318 & -1.174248083 & 0.907640065 \\ \text { H } & 4.672554335 & -0.135784009 & 0.760256052 \\ \text { H } & 5.050490361 & -1.810266129 & 0.312774023 \\ \text { H } & 4.501986326 & -1.424211101 & 1.954858139 \\ \text { C } & 0.724155051 & -2.513472180 & -0.034532001 \\ \text { N } & 0.702170049 & -3.567774254 & -0.458700031 \\ \text { C } & 1.105952081 & -0.304934024 & 5.249305375 \\ \text { O } & 0.395946028 & 0.356590026 & 4.247089307 \\ \text { H } & 0.957038068 & 0.456853033 & 3.487426251 \\ \text { H } & 1.392813099 & -1.311888096 & 4.952254357 \\ \text { H } & 2.003127143 & 0.236234016 & 5.541895398 \\ \text { H } & 0.460765034 & -0.381210030 & 6.113900438\end{array}$

LIIC path connecting the $V^{\prime}\left({ }^{1} \pi \pi^{*}\right) / T_{2}\left({ }^{3} \pi \pi^{*}\right)$ crossing point and $T_{2}\left({ }^{3} \pi \pi^{*}\right)$ minimum Coord_1

$\begin{array}{lrrr}\text { C } & -1.642566117 & -0.658801047 & 0.252363016 \\ \text { C } & -2.093472151 & -1.955012141 & 0.594389042 \\ \text { C } & -2.667149190 & 0.233736019 & -0.198062017 \\ \text { C } & -3.460605252 & -2.423945174 & 0.396059029 \\ \text { C } & -4.036828289 & -0.198727012 & -0.445067034 \\ \text { C } & -4.449777323 & -1.545476110 & -0.170421014 \\ \text { C } & 0.011548002 & 1.214941089 & 0.376948025 \\ \text { C } & -0.425179029 & 2.068644149 & 1.395766098 \\ \text { C } & 0.760502056 & 1.748394127 & -0.666815050 \\ \text { C } & -0.118847008 & 3.426050247 & 1.358163096 \\ \text { C } & 1.079492076 & 3.110302221 & -0.688322052 \\ \text { C } & 0.638320046 & 3.942963284 & 0.311847021 \\ \text { C } & -0.328609023 & -0.252735017 & 0.407157029 \\ \text { H } & -1.428217105 & -2.635256191 & 1.083984076\end{array}$




$\begin{array}{rrrr}\mathrm{H} & -2.423041175 & 1.255134091 & -0.404364027 \\ \mathrm{H} & -3.711658268 & -3.418033243 & 0.706058052 \\ \mathrm{H} & -4.731336339 & 0.504831037 & -0.859517062 \\ \mathrm{H} & -5.445507392 & -1.880557134 & -0.380016026 \\ \mathrm{H} & -0.982418071 & 1.667920121 & 2.222726160 \\ \mathrm{H} & 1.100377077 & 1.112649079 & -1.465892106 \\ \mathrm{H} & -0.460298035 & 4.075056292 & 2.144100152 \\ \mathrm{H} & 1.672070120 & 3.503146250 & -1.494503106 \\ \mathrm{H} & 0.881465064 & 4.990737359 & 0.289158023 \\ \mathrm{C} & 0.781340056 & -1.179360084 & 0.500833034 \\ \mathrm{C} & 2.114904152 & -0.780446059 & 1.044271075 \\ \mathrm{O} & 2.270483163 & 0.004710000 & 1.924887138 \\ \mathrm{O} & 3.093957222 & -1.421618100 & 0.450160031 \\ \mathrm{C} & 4.415108318 & -1.174248083 & 0.907640065 \\ \mathrm{H} & 4.672554335 & -0.135784009 & 0.760256052 \\ \mathrm{H} & 5.050490361 & -1.810266129 & 0.312774023 \\ \mathrm{H} & 4.501986326 & -1.424211101 & 1.954858139 \\ \mathrm{C} & 0.724155051 & -2.513472180 & -0.034532001 \\ \mathrm{~N} & 0.702170049 & -3.567774254 & -0.458700031 \\ \mathrm{C} & 1.105952081 & -0.304934024 & 5.249305375 \\ \mathrm{O} & 0.395946028 & 0.356590026 & 4.247089307 \\ \mathrm{H} & 0.957038068 & 0.456853033 & 3.487426251 \\ \mathrm{H} & 1.392813099 & -1.311888096 & 4.952254357 \\ \mathrm{H} & 2.003127143 & 0.236234016 & 5.541895398 \\ \mathrm{H} & 0.460765034 & -0.381210030 & 6.113900438\end{array}$

\section{Coord 2}

$\begin{array}{lrrr}\text { C } & -1.647392119 & -0.663822050 & 0.250498016 \\ \text { C } & -2.106041153 & -1.948462138 & 0.621054045 \\ \text { C } & -2.654832193 & 0.223356017 & -0.239450017 \\ \text { C } & -3.467144247 & -2.405272176 & 0.424190028 \\ \text { C } & -4.018914290 & -0.204241013 & -0.482486035 \\ \text { C } & -4.440383320 & -1.535052112 & -0.170190013 \\ \text { C } & 0.008754999 & 1.212468085 & 0.390434028 \\ \text { C } & -0.445954030 & 2.061524149 & 1.400994100 \\ \text { C } & 0.774029054 & 1.751636126 & -0.648968045 \\ \text { C } & -0.144375011 & 3.426518246 & 1.369376098 \\ \text { C } & 1.091175076 & 3.121590223 & -0.661143048 \\ \text { C } & 0.633441048 & 3.948259285 & 0.329634023 \\ \text { C } & -0.324161023 & -0.256325019 & 0.412163030 \\ \text { H } & -1.446034106 & -2.621415189 & 1.128122084 \\ \text { H } & -2.397628173 & 1.235529089 & -0.475100033 \\ \text { H } & -3.732106270 & -3.389663242 & 0.754321054 \\ \text { H } & -4.707313337 & 0.490961036 & -0.921199068 \\ \text { H } & -5.438684392 & -1.867490134 & -0.373504029 \\ \text { H } & -1.013700072 & 1.658899120 & 2.219670161 \\ \text { H } & 1.119954079 & 1.119701080 & -1.447927105\end{array}$




$\begin{array}{rrrr}\mathrm{H} & -0.502219034 & 4.073894293 & 2.148737153 \\ \mathrm{H} & 1.695825125 & 3.516090253 & -1.457491105 \\ \mathrm{H} & 0.873882065 & 4.996699361 & 0.314111021 \\ \mathrm{C} & 0.779272058 & -1.180243085 & 0.503010037 \\ \mathrm{C} & 2.117451151 & -0.781034054 & 1.036071077 \\ \mathrm{O} & 2.278929165 & -0.002656999 & 1.921695136 \\ \mathrm{O} & 3.093322225 & -1.413784102 & 0.428123032 \\ \mathrm{C} & 4.417062320 & -1.167683084 & 0.878903063 \\ \mathrm{H} & 4.670540334 & -0.126862011 & 0.741708051 \\ \mathrm{H} & 5.050000364 & -1.794991131 & 0.272336018 \\ \mathrm{H} & 4.512155324 & -1.429480103 & 1.922488140 \\ \mathrm{C} & 0.714075050 & -2.520547179 & -0.016327002 \\ \mathrm{~N} & 0.688814049 & -3.579902256 & -0.427237031 \\ \mathrm{C} & 1.111552078 & -0.302299023 & 5.251516379 \\ \mathrm{O} & 0.403871029 & 0.356353024 & 4.245656306 \\ \mathrm{H} & 0.965587070 & 0.450947034 & 3.485767249 \\ \mathrm{H} & 1.396605098 & -1.311100093 & 4.959068355 \\ \mathrm{H} & 2.009680146 & 0.238217018 & 5.542393396 \\ \mathrm{H} & 0.465482036 & -0.373356028 & 6.115899442\end{array}$

\section{Coord_3}

$\begin{array}{lrrr}\text { C } & -1.652388118 & -0.669002050 & 0.248349016 \\ \text { C } & -2.118728151 & -1.941252140 & 0.647684049 \\ \text { C } & -2.642499188 & 0.211074014 & -0.280802023 \\ \text { C } & -3.473635252 & -2.386226171 & 0.452265035 \\ \text { C } & -4.000998285 & -0.211748017 & -0.519562035 \\ \text { C } & -4.430974321 & -1.525367110 & -0.169786012 \\ \text { C } & 0.006046998 & 1.209710087 & 0.403493027 \\ \text { C } & -0.466172035 & 2.054123150 & 1.405618103 \\ \text { C } & 0.787781059 & 1.754506124 & -0.631239046 \\ \text { C } & -0.169082011 & 3.426591246 & 1.380038099 \\ \text { C } & 1.103292082 & 3.132385227 & -0.633898045 \\ \text { C } & 0.629508044 & 3.953080285 & 0.347338024 \\ \text { C } & -0.319942025 & -0.260041018 & 0.416813033 \\ \text { H } & -1.463881106 & -2.606264188 & 1.172215085 \\ \text { H } & -2.372137171 & 1.212922087 & -0.545678038 \\ \text { H } & -3.752453273 & -3.360227244 & 0.802522058 \\ \text { H } & -4.683330337 & 0.474214032 & -0.982281072 \\ \text { H } & -5.431894392 & -1.854945136 & -0.366665027 \\ \text { H } & -1.044408074 & 1.649671121 & 2.215834161 \\ \text { H } & 1.139586084 & 1.126403083 & -1.430109104 \\ \text { H } & -0.542959041 & 4.072317292 & 2.152607153 \\ \text { H } & 1.719965122 & 3.528449252 & -1.420185104 \\ \text { H } & 0.867420060 & 5.002089360 & 0.339048023 \\ \text { C } & 0.776921056 & -1.181291083 & 0.504934036 \\ \text { C } & 2.119647152 & -0.781884056 & 1.027585074 \\ \text { O } & 2.287071166 & -0.010365000 & 1.918145140\end{array}$




$\begin{array}{lrrr}\mathrm{O} & 3.092052221 & -1.406265102 & 0.405847030 \\ \mathrm{C} & 4.418409320 & -1.161676086 & 0.849854063 \\ \mathrm{H} & 4.668223337 & -0.118680010 & 0.722941050 \\ \mathrm{H} & 5.048627366 & -1.780265129 & 0.231651019 \\ \mathrm{H} & 4.521701327 & -1.435310101 & 1.889594138 \\ \mathrm{C} & 0.703820050 & -2.527621184 & 0.001878002 \\ \mathrm{~N} & 0.675460050 & -3.591862260 & -0.395700030 \\ \mathrm{C} & 1.116767078 & -0.300073023 & 5.253279380 \\ \mathrm{O} & 0.411442032 & 0.355725028 & 4.243792306 \\ \mathrm{H} & 0.973786068 & 0.444669034 & 3.483725249 \\ \mathrm{H} & 1.400005099 & -1.310688092 & 4.965415355 \\ \mathrm{H} & 2.015844145 & 0.239766015 & 5.542491400 \\ \mathrm{H} & 0.469801032 & -0.365934027 & 6.117406439\end{array}$

\section{Coord_4}

$\begin{array}{lrrr}\text { C } & -1.657536118 & -0.674360049 & 0.245911017 \\ \text { C } & -2.131518154 & -1.933371141 & 0.674249049 \\ \text { C } & -2.630158187 & 0.196872013 & -0.322060025 \\ \text { C } & -3.480075249 & -2.366781172 & 0.480255035 \\ \text { C } & -3.983094287 & -0.221237017 & -0.556242041 \\ \text { C } & -4.421554316 & -1.516394111 & -0.169205013 \\ \text { C } & 0.003424999 & 1.206667085 & 0.416125028 \\ \text { C } & -0.485851036 & 2.046451149 & 1.409639099 \\ \text { C } & 0.801752058 & 1.757001126 & -0.613628043 \\ \text { C } & -0.193003013 & 3.426290245 & 1.390146098 \\ \text { C } & 1.115816083 & 3.142687229 & -0.606589044 \\ \text { C } & 0.626473043 & 3.957438282 & 0.364957028 \\ \text { C } & -0.315936021 & -0.263890021 & 0.421109031 \\ \text { H } & -1.481744108 & -2.589788186 & 1.216209087 \\ \text { H } & -2.346586170 & 1.187284084 & -0.615999043 \\ \text { H } & -3.772695271 & -3.329687239 & 0.850611062 \\ \text { H } & -4.659411334 & 0.454610031 & -1.042679077 \\ \text { H } & -5.425142392 & -1.842871131 & -0.359508026 \\ \text { H } & -1.074554079 & 1.640251120 & 2.211221159 \\ \text { H } & 1.159280083 & 1.132753083 & -1.412433102 \\ \text { H } & -0.582560041 & 4.070356293 & 2.155710153 \\ \text { H } & 1.744456127 & 3.540219254 & -1.382590098 \\ \text { H } & 0.862015065 & 5.006925362 & 0.363965027 \\ \text { C } & 0.774308058 & -1.182512086 & 0.506610035 \\ \text { C } & 2.121513153 & -0.783002054 & 1.018825074 \\ \text { O } & 2.294920166 & -0.018423999 & 1.914253136 \\ \text { O } & 3.090171223 & -1.399065100 & 0.383349028 \\ \text { C } & 4.419171319 & -1.156228084 & 0.820511058 \\ \text { H } & 4.665626336 & -0.111238006 & 0.703968050 \\ \text { H } & 5.046400366 & -1.766089126 & 0.190743014 \\ \text { H } & 4.530638326 & -1.441704102 & 1.856194131 \\ \text { C } & 0.693421050 & -2.534695183 & 0.020083002\end{array}$




$\begin{array}{rrrr}\mathrm{N} & 0.662109045 & -3.603655261 & -0.364093024 \\ \mathrm{C} & 1.121578082 & -0.298276022 & 5.254601376 \\ \mathrm{O} & 0.418648029 & 0.354686025 & 4.241499306 \\ \mathrm{H} & 0.981632073 & 0.438003031 & 3.481307253 \\ \mathrm{H} & 1.402998099 & -1.310674095 & 4.971302356 \\ \mathrm{H} & 2.021594148 & 0.240860015 & 5.542205401 \\ \mathrm{H} & 0.473693036 & -0.358968027 & 6.118421438\end{array}$

$\begin{array}{lrrr}\text { Coord_5 } & & & \\ \mathrm{C} & -1.662816122 & -0.679919051 & 0.243180018 \\ \mathrm{C} & -2.144397153 & -1.924804137 & 0.700716050 \\ \mathrm{C} & -2.617816186 & 0.180733012 & -0.363164027 \\ \mathrm{C} & -3.486459248 & -2.346915170 & 0.508131039 \\ \mathrm{C} & -3.965217283 & -0.232693016 & -0.592470044 \\ \mathrm{C} & -4.412127319 & -1.508103110 & -0.168441013 \\ \mathrm{C} & 0.000887002 & 1.203339089 & 0.428328030 \\ \mathrm{C} & -0.505009036 & 2.038521147 & 1.413058103 \\ \mathrm{C} & 0.815930060 & 1.759116126 & -0.596133041 \\ \mathrm{C} & -0.216169017 & 3.425631244 & 1.399698102 \\ \mathrm{C} & 1.128720081 & 3.152493227 & -0.579221044 \\ \mathrm{C} & 0.624293044 & 3.961342287 & 0.382490030 \\ \mathrm{C} & -0.312126024 & -0.267885018 & 0.425053031 \\ \mathrm{H} & -1.499607106 & -2.571971185 & 1.260048089 \\ \mathrm{H} & -2.320993169 & 1.158589083 & -0.685965049 \\ \mathrm{H} & -3.792824274 & -3.298009239 & 0.898539063 \\ \mathrm{H} & -4.635578334 & 0.432165031 & -1.102307082 \\ \mathrm{H} & -5.418431387 & -1.831222130 & -0.352038028 \\ \mathrm{H} & -1.104147079 & 1.630654115 & 2.205835161 \\ \mathrm{H} & 1.179040085 & 1.138743084 & -1.394897099 \\ \mathrm{H} & -0.621062047 & 4.068042291 & 2.158047153 \\ \mathrm{H} & 1.769261126 & 3.551394255 & -1.344714099 \\ \mathrm{H} & 0.857607060 & 5.011217360 & 0.388857027 \\ \mathrm{C} & 0.771453057 & -1.183912088 & 0.508042037 \\ \mathrm{C} & 2.123065150 & -0.784392054 & 1.009803073 \\ \mathrm{O} & 2.302485168 & -0.026843003 & 1.910033138 \\ \mathrm{O} & 3.087702220 & -1.392187098 & 0.360646027 \\ \mathrm{C} & 4.419369316 & -1.151338085 & 0.790891055 \\ \mathrm{H} & 4.662772335 & -0.104534007 & 0.684802050 \\ \mathrm{H} & 5.043346362 & -1.752461124 & 0.149632011 \\ \mathrm{H} & 4.538976329 & -1.448662106 & 1.822301133 \\ \mathrm{C} & 0.682905050 & -2.541768182 & 0.038289001 \\ \mathrm{~N} & 0.648762047 & -3.615277258 & -0.332419025 \\ \mathrm{C} & 1.125962083 & -0.296930022 & 5.255486377 \\ \mathrm{O} & 0.425475029 & 0.353216024 & 4.238775303 \\ \mathrm{H} & 0.989117074 & 0.430933032 & 3.478516250 \\ \mathrm{H} & 1.405567101 & -1.311077095 & 4.976730360 \\ \mathrm{H} & 2.026908146 & 0.241480015 & 5.541548401\end{array}$


Coord_6

\begin{tabular}{|c|c|c|c|}
\hline & -1.668213121 & -0.685697047 & 0.240156010 \\
\hline & -2.157350153 & -1.915539137 & 0.727054053 \\
\hline & -2.605484186 & 0.162643014 & -0.404054029 \\
\hline & -3.492778249 & -2.326602167 & 0.535865039 \\
\hline & -3.947380284 & -0.246106019 & -0.628191043 \\
\hline & -4.402697317 & -1.500469109 & -0 \\
\hline & -0.001570000 & 1.199724089 & 0.440104034 \\
\hline & -0.523660036 & 2.030342147 & 1.415877104 \\
\hline & 0.830307060 & 1.760846128 & -0.578755040 \\
\hline & -0.238609017 & 3.424630248 & 1.408695100 \\
\hline & 1.141975082 & 3.161802227 & -0.001190041 \\
\hline & 0.622925047 & 3.964799286 & 0.399939031 \\
\hline & -0.308495022 & -0.272033021 & 0.428648029 \\
\hline & -1.517452111 & -2.552799183 & 1.303675096 \\
\hline & -2.295380164 & 1.126812079 & -0.755475052 \\
\hline & -3.812831275 & -3.265158233 & 0.946254066 \\
\hline & -4.611859334 & 0.406900029 & -1.161079084 \\
\hline & -5.411762389 & -1.819950131 & -0.344262027 \\
\hline & -1.133197084 & 1.620892118 & 2.199681158 \\
\hline & 1.198868087 & 1.144371080 & -1.377496100 \\
\hline & -0.658501046 & 4.065398294 & 2.159620154 \\
\hline & 1.794346128 & 3.561968254 & -1.306563092 \\
\hline & 8060 & 5.014978361 & 0.413718028 \\
\hline & 0.768375055 & -1.185496084 & 0.509234035 \\
\hline & 2.124320152 & -0.786059058 & 1.000529072 \\
\hline & 2.309774166 & -0.035631002 & 1.905498137 \\
\hline & 3.084667224 & -1.38 & 0.337752025 \\
\hline & 4.419021319 & -1.147007081 & 0.761009056 \\
\hline & 4.659682337 & -0.098568005 & 0.665455049 \\
\hline & 5.039490364 & -1.739379127 & 0.108340009 \\
\hline & 4.546724325 & -1.456184106 & 1.787932129 \\
\hline & 0.672298046 & -2.548842181 & 0.056494005 \\
\hline & 0.635419043 & -3.626730262 & -0.300681021 \\
\hline & 1.129900082 & -0.296054022 & 5.255936379 \\
\hline & 0.431910031 & 0.351296026 & 4.235620306 \\
\hline & 0.996235074 & 0.423444031 & 3.475356253 \\
\hline & 1.407696103 & -1.311918096 & 4.9817033 \\
\hline & 2.031760146 & 0.241608018 & 5.540534397 \\
\hline & & & \\
\hline
\end{tabular}

Coord_7

$\begin{array}{lrrr}C & -1.673711120 & -0.691716052 & 0.236836019 \\ \text { C } & -2.170359155 & -1.905565136 & 0.753228055 \\ \text { C } & -2.593172185 & 0.142587011 & -0.444667034\end{array}$




\begin{tabular}{|c|c|c|c|}
\hline & J & -2.305822165 & 0.563428040 \\
\hline$C$ & -3.929600283 & -0.261460017 & \\
\hline$C$ & -4.393266315 & -1.493466109 & -0.1663 \\
\hline $\mathrm{U}$ & -0.003946001 & 1.195822084 & 0.45145203 \\
\hline U & -0.541819039 & 2.021921148 & .418100104 \\
\hline & 0.844871060 & 1.762186127 & -0.5014950 \\
\hline$C$ & -0.260352021 & 3.423299245 & 1.417134 \\
\hline 0 & 5554082 & 3.170612230 & -0.5243170 \\
\hline U & 622327045 & 3.967813284 & 0.417301029 \\
\hline & -0.305030022 & -0.276346022 & \\
\hline & -1.535260110 & -2.532256184 & $1.34 / 0340$ \\
\hline & -2.269774161 & 1.091932080 & -0.8244270 \\
\hline & -3.832705278 & -3.231099232 & 0.993705072 \\
\hline & -4.588279329 & 0.378836029 & -1.218908087 \\
\hline & -5.40 & -1.80 & -0.3 \\
\hline$\Pi$ & -1.161712086 & 1.610974114 & 2.1927651 \\
\hline & 1.218765088 & 1.14963 & -1.360227099 \\
\hline & -0.694914050 & 45294 & 2.1 \\
\hline & 1.8 & 3.57 & -1.26 \\
\hline & 0.851550061 & 5.018215359 & 0.43854603 \\
\hline c & 0.765093055 & -1.187269087 & 0.51019103 \\
\hline 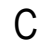 & 2.12 & & \\
\hline 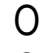 & & & \\
\hline 0 & 3.08 & -1. & 0.3 \\
\hline$C$ & 4.418 & -1.143 & 0.7308800 \\
\hline H & 4.656 & $-0.0 s$ & 0.6 \\
\hline 11 & & & \\
\hline & 4.553 & -1.46 & 1.7 \\
\hline C & 0.661626049 & -2.55591 & 0.0746990 \\
\hline$N$ & 0.622 & -3.63801 & -0.268883020 \\
\hline 乙 & 33 & -0.2 & 5. \\
\hline 0 & 0.43 & & 4.2 \\
\hline$H$ & 1.0029 & 0.415520030 & 3.47 \\
\hline$H_{2}$ & 1.409366102 & -1.313214093 & 4.986223 \\
\hline$H$ & 2.03 & & \\
\hline & & -0.341056023 & 6.1184 \\
\hline \multicolumn{4}{|c|}{ Coord_8 } \\
\hline C & -1.67 & -0.6 & 0.23 \\
\hline C & -2.18 & -1.894868135 & 0.779207054 \\
\hline$C$ & -2.580893187 & 0.120556007 & -0.4849400 \\
\hline C & -3.505189250 & -2.284552166 & 0.5907880 \\
\hline C & -3.911895283 & -0.278739020 & -0.6978870 \\
\hline C & -4.383836318 & -1.487068108 & -0.164999011 \\
\hline 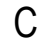 & -0.00 & 1.19163 & 0.46237 \\
\hline C & & 2.013265147 & 1.419731 \\
\hline & & 1.76313 & -0.5443 \\
\hline
\end{tabular}




\begin{tabular}{|c|c|c|c|}
\hline & 018 & 244 & 1.425018101 \\
\hline C & 1.169429083 & 3.178918227 & \\
\hline$C$ & 0.622457043 & 3.970390287 & 0.4345790 \\
\hline 0 & -0.301718023 & -0.280832022 & 0.43480403 \\
\hline$\Pi$ & -1.553012113 & -2.510329179 & .39006610 \\
\hline & -2.244200162 & 1.053933074 & \\
\hline & -3.852434276 & -3.195802231 & 1.0408 \\
\hline & -4.564868326 & 0.347996024 & -1.2757060 \\
\hline & -5.398551389 & -1.798364128 & -0.327830026 \\
\hline & 698085 & 1.600909115 & \\
\hline & 1.238732088 & 1.154519085 & -1.3430 \\
\hline & -0.730333053 & 4.059204290 & 2.16048 \\
\hline & 1.845211132 & 3.581287256 & -1.229459088 \\
\hline & 0.849787059 & 5.020935362 & 0.463337032 \\
\hline C & 21054 & -1.18 & 0.5 \\
\hline C & 2.126000153 & -0.790244056 & 0.981265 \\
\hline 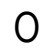 & 2.323554170 & -0.054345006 & 1.8955411 \\
\hline & 3.076982221 & -1.373496097 & 0.2 \\
\hline & 3319 & -1.14 & \\
\hline П & 4.652869333 & -0.088850008 & 0.626256 \\
\hline$\Pi$ & 5.029465360 & -1.714848126 & 0.0252830 \\
\hline & & & \\
\hline & & & \\
\hline & 0.6 & -3.64 & -0.2 \\
\hline $\mathrm{C}$ & 1.136 & -0.29579 & 5.2555 \\
\hline U & 0.443 & 0.34 & 4.2 \\
\hline & & & \\
\hline & 1.41 & -1.3 & 4.9 \\
\hline H & 2.039 & 15 & 5.53 \\
\hline & & & \\
\hline \multicolumn{4}{|c|}{ Coord_9 } \\
\hline C & -1.684950119 & -0.704561052 & 0.2293110 \\
\hline C & -2.196487160 & -1.883439134 & 0.8049540 \\
\hline C & & 0.0965 & -0.52480 \\
\hline C & -3.5 & -2.2627 & 0.6 \\
\hline C & -3.89 & -0.297928019 & -0.7317470 \\
\hline C & -4.374410317 & -1.481250106 & -0.1634500 \\
\hline C & -0.008469000 & 1.187146085 & 0.472873 \\
\hline C & & 2.00 & $1.4 t$ \\
\hline C & & 1.76367 & -0.5273090 \\
\hline C & -0.30 & 3.419691246 & 1.432347 \\
\hline C & 1.183572086 & 3.186720232 & -0.4692090 \\
\hline C & 0.623276 & 3.972533285 & 0.4517730 \\
\hline C & -0.298546024 & -0.285501021 & 0.43737 \\
\hline $\mathrm{H}$ & -1.570687114 & -2.487007179 & 1.43271 \\
\hline & & & -0.9 \\
\hline
\end{tabular}

S52 


$\begin{array}{rrrr}\mathrm{H} & -3.872003277 & -3.159236226 & 1.087600077 \\ \mathrm{H} & -4.541653325 & 0.314408022 & -1.331384098 \\ \mathrm{H} & -5.392008387 & -1.787964128 & -0.319193023 \\ \mathrm{H} & -1.217165088 & 1.590704117 & 2.176673155 \\ \mathrm{H} & 1.258765093 & 1.159029083 & -1.326066094 \\ \mathrm{H} & -0.764791054 & 4.055691294 & 2.159779156 \\ \mathrm{H} & 1.870918134 & 3.590021258 & -1.190521088 \\ \mathrm{H} & 0.848796063 & 5.023144359 & 0.488086038 \\ \mathrm{C} & 0.757976055 & -1.191405084 & 0.511420034 \\ \mathrm{C} & 2.126453155 & -0.792769057 & 0.971297072 \\ \mathrm{O} & 2.330056165 & -0.064287003 & 1.890144138 \\ \mathrm{O} & 3.072369219 & -1.367919098 & 0.268076019 \\ \mathrm{C} & 4.414884317 & -1.137361079 & 0.669927046 \\ \mathrm{H} & 4.649180333 & -0.085096004 & 0.606418045 \\ \mathrm{H} & 5.023341361 & -1.703393121 & -0.016449004 \\ \mathrm{H} & 4.566522328 & -1.482131106 & 1.682089123 \\ \mathrm{C} & 0.640179046 & -2.570064184 & 0.111109008 \\ \mathrm{~N} & 0.595425044 & -3.660048262 & -0.205118013 \\ \mathrm{C} & 1.138802082 & -0.296460023 & 5.254713376 \\ \mathrm{O} & 0.448715033 & 0.342646026 & 4.223552302 \\ \mathrm{H} & 1.015302071 & 0.398303026 & 3.463693249 \\ \mathrm{H} & 1.411249100 & -1.317258097 & 4.993913359 \\ \mathrm{H} & 2.043273148 & 0.238847019 & 5.535470398 \\ \mathrm{H} & 0.485743037 & -0.331847026 & 6.116051438\end{array}$

\begin{tabular}{lrrr}
\multicolumn{4}{l}{ Coord_10 } \\
C & -1.709936123 & -0.669024048 & 0.194563012 \\
C & -2.245596163 & -1.823858131 & 0.794847057 \\
C & -2.562954185 & 0.125829010 & -0.595818043 \\
C & -3.557087255 & -2.176119154 & 0.603381044 \\
C & -3.887260277 & -0.246572018 & -0.802401057 \\
C & -4.392184317 & -1.398926101 & -0.204146015 \\
C & -0.007244003 & 1.202845086 & 0.463695036 \\
C & -0.583352040 & 2.020499143 & 1.402012099 \\
C & 0.903855067 & 1.775581127 & -0.524747035 \\
C & -0.293794023 & 3.439093249 & 1.424739103 \\
C & 1.229893087 & 3.201601230 & -0.450956030 \\
C & 0.663134046 & 3.986550286 & 0.459496036 \\
C & -0.310412025 & -0.266072019 & 0.415335030 \\
H & -1.634149119 & -2.424327176 & 1.440008105 \\
H & -2.186775159 & 1.020415072 & -1.054726078 \\
H & -3.944085283 & -3.053552221 & 1.088776078 \\
H & -4.519297328 & 0.360188024 & -1.424128104 \\
H & -5.415843388 & -1.687483122 & -0.357314025 \\
H & -1.241903088 & 1.611825116 & 2.144771154 \\
H & 1.288549094 & 1.172198082 & -1.323704095 \\
H & -0.765111052 & 4.077607292 & 2.143889153
\end{tabular}




$\begin{array}{rrrr}\mathrm{H} & 1.936347138 & 3.598796260 & -1.157033085 \\ \mathrm{H} & 0.899909066 & 5.034142361 & 0.507058037 \\ \mathrm{C} & 0.727576050 & -1.182781088 & 0.488858033 \\ \mathrm{C} & 2.103276149 & -0.803042060 & 0.944398067 \\ \mathrm{O} & 2.318508168 & -0.087221005 & 1.870472133 \\ \mathrm{O} & 3.039344220 & -1.379986102 & 0.229789018 \\ \mathrm{C} & 4.385875317 & -1.170523083 & 0.629914044 \\ \mathrm{H} & 4.632059332 & -0.120212010 & 0.580915041 \\ \mathrm{H} & 4.985402358 & -1.733428127 & -0.066780004 \\ \mathrm{H} & 4.537119324 & -1.531808113 & 1.636336119 \\ \mathrm{C} & 0.586932040 & -2.563449182 & 0.102287009 \\ \mathrm{~N} & 0.527003040 & -3.655627261 & -0.203302014 \\ \mathrm{C} & 1.107701081 & -0.304165024 & 5.234401379 \\ \mathrm{O} & 0.432300029 & 0.343559026 & 4.198798301 \\ \mathrm{H} & 1.003139070 & 0.388689029 & 3.441463249 \\ \mathrm{H} & 1.366513101 & -1.329169094 & 4.976276358 \\ \mathrm{H} & 2.018574144 & 0.218177017 & 5.518867398 \\ \mathrm{H} & 0.449913032 & -0.328692024 & 6.092514438\end{array}$

LIIC path connecting the $T_{2}\left({ }^{3} \pi \pi^{*}\right)$ minimum and $T_{2}\left({ }^{3} \pi \pi^{*}\right) / T_{1}\left({ }^{3} \pi \pi^{*}\right)$ conical intersection Coord_1
C $\quad-1.709936123 \quad-0.669024048$
0.194563012
C $\quad-2.245596163-1.823858131$
0.794847057
C -2.562954185
0.125829010
$-0.595818043$
C $\quad-3.557087255$
$-2.176119154$
0.603381044
C -3.887260277
$-0.246572018$
$-0.802401057$
C $\quad-4.392184317 \quad-1.398926101$
$-0.204146015$
C -0.007244003
1.202845086
0.463695036
C -0.583352040
2.020499143
1.402012099
C $\quad 0.903855067$
1.775581127
$-0.524747035$
C $\quad-0.293794023$
3.439093249
1.424739103
C $\quad 1.229893087$
3.201601230
$-0.450956030$
C $\quad 0.663134046$
3.986550286
0.459496036
C $\quad-0.310412025$
$-0.266072019$
0.415335030
H $\quad-1.634149119$
$-2.424327176$
1.440008105
H $\quad-2.186775159$
1.020415072
$-1.054726078$
$\mathrm{H} \quad-3.944085283$
$-3.053552221$
1.088776078
H $\quad-4.519297328$
0.360188024
$-1.424128104$
H $\quad-5.415843388$
$-1.687483122$
$-0.357314025$
H $\quad-1.241903088$
1.611825116
2.144771154
H $\quad 1.288549094$
1.172198082
$-1.323704095$
H $\quad-0.765111052$
4.077607292
2.143889153
H $\quad 1.936347138$
3.598796260
$-1.157033085$
H 0.899909066
5.034142361
0.507058037
$-1.182781088$
0.488858033
C $\quad 2.103276149$
$-0.803042060$
0.944398067
O 2.318508168
$-0.087221005$
1.870472133 


$\begin{array}{lrrr}\mathrm{O} & 3.039344220 & -1.379986102 & 0.229789018 \\ \mathrm{C} & 4.385875317 & -1.170523083 & 0.629914044 \\ \mathrm{H} & 4.632059332 & -0.120212010 & 0.580915041 \\ \mathrm{H} & 4.985402358 & -1.733428127 & -0.066780004 \\ \mathrm{H} & 4.537119324 & -1.531808113 & 1.636336119 \\ \mathrm{C} & 0.586932040 & -2.563449182 & 0.102287009 \\ \mathrm{~N} & 0.527003040 & -3.655627261 & -0.203302014 \\ \mathrm{C} & 1.107701081 & -0.304165024 & 5.234401379 \\ \mathrm{O} & 0.432300029 & 0.343559026 & 4.198798301 \\ \mathrm{H} & 1.003139070 & 0.388689029 & 3.441463249 \\ \mathrm{H} & 1.366513101 & -1.329169094 & 4.976276358 \\ \mathrm{H} & 2.018574144 & 0.218177017 & 5.518867398 \\ \mathrm{H} & 0.449913032 & -0.328692024 & 6.092514438\end{array}$

\section{Coord_2}

$\begin{array}{lrrr}\text { C } & -1.709843125 & -0.669026048 & 0.194542014 \\ \text { C } & -2.245209160 & -1.823387132 & 0.795496056 \\ \text { C } & -2.562759183 & 0.125378008 & -0.595981045 \\ \text { C } & -3.556791255 & -2.175915156 & 0.604261045 \\ \text { C } & -3.887115277 & -0.247229018 & -0.802407058 \\ \text { C } & -4.391869313 & -1.399238099 & -0.203546013 \\ \text { C } & -0.007220999 & 1.202920087 & 0.464244031 \\ \text { C } & -0.582670041 & 2.020260145 & 1.402754101 \\ \text { C } & 0.903463063 & 1.775634129 & -0.525177039 \\ \text { C } & -0.293062022 & 3.439662247 & 1.425195100 \\ \text { C } & 1.229936088 & 3.202377232 & -0.451352035 \\ \text { C } & 0.663901046 & 3.987120289 & 0.458968033 \\ \text { C } & -0.309887023 & -0.266297020 & 0.415448030 \\ \text { H } & -1.633614116 & -2.423358173 & 1.440986104 \\ \text { H } & -2.186746155 & 1.019855075 & -1.055243073 \\ \text { H } & -3.943661285 & -3.053150221 & 1.090091078 \\ \text { H } & -4.519193323 & 0.359143026 & -1.424468101 \\ \text { H } & -5.415526389 & -1.687921122 & -0.356513026 \\ \text { H } & -1.240790090 & 1.611671114 & 2.145941155 \\ \text { H } & 1.287754095 & 1.172183085 & -1.324227097 \\ \text { H } & -0.764318054 & 4.078253295 & 2.144262155 \\ \text { H } & 1.936177140 & 3.599344260 & -1.157749083 \\ \text { H } & 0.900786066 & 5.034687361 & 0.506322036 \\ \text { C } & 0.727438051 & -1.182273083 & 0.488781033 \\ \text { C } & 2.103352149 & -0.802748059 & 0.944216067 \\ \text { O } & 2.318758167 & -0.087174009 & 1.870453135 \\ \text { O } & 3.039368218 & -1.379575100 & 0.229464019 \\ \text { C } & 4.385903316 & -1.170398086 & 0.629753047 \\ \text { H } & 4.632195336 & -0.120091009 & 0.581167040 \\ \text { H } & 4.985423361 & -1.733140127 & -0.067075005 \\ \text { H } & 4.536988326 & -1.532036109 & 1.636077118 \\ \text { C } & 0.586791041 & -2.563103185 & 0.102203008\end{array}$




$\begin{array}{rrrr}\mathrm{N} & 0.526899040 & -3.655209264 & -0.203506012 \\ \mathrm{C} & 1.106360082 & -0.304828025 & 5.233954377 \\ \mathrm{O} & 0.431466030 & 0.342802022 & 4.197955300 \\ \mathrm{H} & 1.002744071 & 0.387955029 & 3.440942247 \\ \mathrm{H} & 1.365635100 & -1.329727095 & 4.975882359 \\ \mathrm{H} & 2.016886143 & 0.217764016 & 5.519075397 \\ \mathrm{H} & 0.447993035 & -0.329627022 & 6.091615440\end{array}$

\section{Coord_3}

$\begin{array}{lrrr}\text { C } & -1.709748122 & -0.669027048 & 0.194520016 \\ \mathrm{C} & -2.244821162 & -1.822914132 & 0.796144060 \\ \mathrm{C} & -2.562564186 & 0.124927011 & -0.596144042 \\ \mathrm{C} & -3.556494254 & -2.175709158 & 0.605141045 \\ \mathrm{C} & -3.886969277 & -0.247886018 & -0.802412058 \\ \mathrm{C} & -4.391551315 & -1.399548102 & -0.202944015 \\ \mathrm{C} & -0.007196001 & 1.202994087 & 0.464793031 \\ \mathrm{C} & -0.581988043 & 2.020020147 & 1.403494102 \\ \mathrm{C} & 0.903070064 & 1.775686126 & -0.525605038 \\ \mathrm{C} & -0.292330022 & 3.440229250 & 1.425650103 \\ \mathrm{C} & 1.229977089 & 3.203152233 & -0.451747034 \\ \mathrm{C} & 0.664666046 & 3.987688287 & 0.458440031 \\ \mathrm{C} & -0.309361020 & -0.266522021 & 0.415562031 \\ \mathrm{H} & -1.633077117 & -2.422388176 & 1.441964103 \\ \mathrm{H} & -2.186716156 & 1.019294073 & -1.055759074 \\ \mathrm{H} & -3.943235281 & -3.052746221 & 1.091406078 \\ \mathrm{H} & -4.519088324 & 0.358099028 & -1.424807102 \\ \mathrm{H} & -5.415207391 & -1.688358121 & -0.355710026 \\ \mathrm{H} & -1.239677088 & 1.611516118 & 2.147109155 \\ \mathrm{H} & 1.286959091 & 1.172168083 & -1.324749093 \\ \mathrm{H} & -0.763527056 & 4.078897293 & 2.144633156 \\ \mathrm{H} & 1.936006142 & 3.599891260 & -1.158463086 \\ \mathrm{H} & 0.901660066 & 5.035230360 & 0.505585035 \\ \mathrm{C} & 0.727300052 & -1.181763083 & 0.488705037 \\ \mathrm{C} & 2.103427150 & -0.802455059 & 0.944034067 \\ \mathrm{O} & 2.319007166 & -0.087126007 & 1.870433132 \\ \mathrm{O} & 3.039390221 & -1.379164099 & 0.229141014 \\ \mathrm{C} & 4.385929315 & -1.170274084 & 0.629593045 \\ \mathrm{H} & 4.632330334 & -0.119970007 & 0.581419040 \\ \mathrm{H} & 4.985442359 & -1.732852122 & -0.067369005 \\ \mathrm{H} & 4.536856328 & -1.532265110 & 1.635818118 \\ \mathrm{C} & 0.586651042 & -2.562757182 & 0.102118006 \\ \mathrm{~N} & 0.526794036 & -3.654790262 & -0.203710015 \\ \mathrm{C} & 1.105019078 & -0.305491020 & 5.233504376 \\ \mathrm{O} & 0.430632031 & 0.342045023 & 4.197110300 \\ \mathrm{H} & 1.002349071 & 0.387220028 & 3.440419246 \\ \mathrm{H} & 1.364756100 & -1.330284097 & 4.975485360 \\ \mathrm{H} & 2.015197147 & 0.217350014 & 5.519279400 \\ & & & \\ & & & \end{array}$




\section{$\begin{array}{llll}H & 0.446072031 & -0.330562025 & 6.090713437\end{array}$}

Coord_4

\begin{tabular}{|c|c|c|c|}
\hline & -1.709653124 & -0.669028048 & 0.194 \\
\hline & -2.244432164 & -1.822441132 & 0.796791058 \\
\hline & -2.562367184 & 0.124477010 & -0.596307045 \\
\hline & -3.556196258 & -2.175502155 & 0.606022046 \\
\hline C & -3.886822277 & -0.248542018 & -0.802416059 \\
\hline & -4.391234317 & -1.399857099 & 42013 \\
\hline & -0.007171003 & 1.203069087 & 0.465343031 \\
\hline & -0.581307040 & 2.019780144 & 1.404234099 \\
\hline & 0.902677066 & 1.775739128 & -0.526034037 \\
\hline & -0.291600022 & 3.440796247 & 1.426105100 \\
\hline & 1.230018089 & 3.203926229 & -0.452142033 \\
\hline & 0.665429050 & 3.988256285 & 0.457912033 \\
\hline & -0.308835023 & -0.266747017 & 0.415675032 \\
\hline & -1.632540119 & -2.421416172 & 1.442940102 \\
\hline & -2.186686157 & 1.018733071 & -1.056276075 \\
\hline & -3.942809283 & -3.052341220 & 1.092722078 \\
\hline & -4.518982324 & 0.357055025 & -1.425144104 \\
\hline & -5.414887392 & -1.688794121 & -0.354906026 \\
\hline & -1.238563090 & 1.611361117 & 2.148277155 \\
\hline & 1.286164092 & 1.172153086 & -1.325270095 \\
\hline & -0.762737057 & 4.079541291 & 2.145004156 \\
\hline & 1.935835138 & 3.600438260 & -1.159177083 \\
\hline & 0.902 & 5.035 & 0.504847034 \\
\hline & 0.727163053 & -1.181254083 & 0.488629037 \\
\hline & 2.103502150 & -0.802161059 & 0.943853067 \\
\hline & 2.319257166 & -0.087079006 & 1.870414135 \\
\hline & 3.039413219 & -1.378754098 & 0.228818015 \\
\hline & 4.385955313 & -1.170149082 & 0.629434043 \\
\hline & 4.632466333 & -0.119850011 & 0.581673040 \\
\hline & 4.985462356 & -1.732565123 & -0.067662005 \\
\hline & 4.536723325 & -1.532494112 & 1.635559117 \\
\hline & 0.586510043 & -2.562411185 & 0.102034010 \\
\hline & 0.526689036 & -3.654372264 & -0.203914013 \\
\hline & 1.103677079 & -0.306154021 & 5.233053379 \\
\hline & 0.429798032 & 0.341289025 & 4.196264304 \\
\hline & 1.001954072 & 0.386485027 & 3.439897249 \\
\hline & 1.363876099 & -1.330842098 & 4.975088360 \\
\hline & 2.013507145 & 0.216935017 & 5.519483398 \\
\hline & & & \\
\hline
\end{tabular}

Coord_5

$\begin{array}{lrrr}C & -1.709558121 & -0.669029048 & 0.194477015 \\ C & -2.244042160 & -1.821967132 & 0.797439057 \\ C & -2.562171182 & 0.124027008 & -0.596469042\end{array}$




\begin{tabular}{|c|c|c|c|}
\hline & 7257 & -2175294156 & \\
\hline$C$ & -3.886675282 & .249198018 & \\
\hline$C$ & -4.390916318 & -1.400166101 & -0.201738015 \\
\hline & -0.007145999 & 1.203143087 & 65894032 \\
\hline C & 80625041 & 2.019539146 & 74100 \\
\hline C & 02284067 & 1.775791125 & \\
\hline 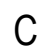 & 290871022 & 3.441363250 & 1.4265591 \\
\hline & 30059090 & 3.204700230 & -0.452536032 \\
\hline & 66192050 & 3.988823287 & 0.457382031 \\
\hline C & -0.308308021 & -0.266971018 & 0.41 \\
\hline & -1.632002115 & -2.420443174 & 1.4439 \\
\hline & -2.186656158 & 1.018172074 & -1.056792 \\
\hline & -3.942381284 & -3.051934219 & 1.09403907 \\
\hline & -4.5 & 0.35 & \\
\hline & -5.41 & -1.689 & -0.3 \\
\hline$\Pi$ & -1.237449087 & 1.611206116 & 2.149443154 \\
\hline & 1.285369093 & 1.172138084 & -1.325790096 \\
\hline & -0.76 & 4.080185295 & \\
\hline & 1.93 & 3.600 & -1.15 \\
\hline $\mathrm{H}$ & 0.9034 & 5.036315364 & 0.504108038 \\
\hline C & 0.727025055 & -1.180745083 & 0.488553037 \\
\hline 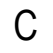 & & -0.8 & \\
\hline 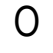 & 2.31 & -0.0 & 1.87 \\
\hline 0 & 3.03 & -1.3 & 0.228495016 \\
\hline C & 4.385981317 & -1.170026085 & 0.629275047 \\
\hline П & 4.632 & -0.11 & 0.5 \\
\hline & & & \\
\hline & 4.53 & -1.53 & 1.6 \\
\hline C & 0.586369043 & -2.562065183 & 0.101949008 \\
\hline N & 0.5265 & -3.653953262 & -0.204118016 \\
\hline 乙 & & -0.3 & \\
\hline 0 & 0.42 & 0.3 & 4.1 \\
\hline$H$ & 1.0015 & 0.385750026 & 3.439373247 \\
\hline$H_{2}$ & 1.3629 & -1.331400095 & 4.974690356 \\
\hline$H$ & & & \\
\hline & & -0.332430026 & 6.088905439 \\
\hline \multicolumn{4}{|c|}{ Coord_6 } \\
\hline$c$ & -1.70 & -0.6 & \\
\hline C & -2.243652162 & -1.821492132 & 0.798086055 \\
\hline C & -2.5 & 0.12 & -0.5966320 \\
\hline C & -3.555597256 & -2.175086158 & 0.607785042 \\
\hline C & -3.886528282 & -0.249854017 & -0.802421060 \\
\hline C & -4.390597314 & -1.400474098 & -0.201134012 \\
\hline 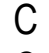 & & & 0.466444032 \\
\hline C & & 2.019299143 & 1.405713101 \\
\hline & & 1.775843128 & \\
\hline
\end{tabular}

S58 


\begin{tabular}{|c|c|c|c|}
\hline & 3022 & 30248 & 1.427012105 \\
\hline 0 & 1.230099091 & 3.205474231 & \\
\hline$C$ & 66952049 & 3.989391285 & 0.4568520 \\
\hline & -0.307780024 & -0.267195018 & 0.41590202 \\
\hline$\Pi$ & -1.631463116 & -2.419469176 & 44480110 \\
\hline & -2.186626159 & 1.017612073 & -1.051 \\
\hline & 952286 & -3.051527219 & \\
\hline & -4.518771325 & 0.354967024 & -1.42581510 \\
\hline & -5.414246389 & -1.689664120 & -0.353293025 \\
\hline & -1.236336090 & 1.611051115 & \\
\hline & 1.284575094 & 1.172122082 & $-1.3<63$ \\
\hline & -0.761163057 & 4.080829293 & 2.1457421 \\
\hline & 1.935491141 & 3.601532260 & -1.160604084 \\
\hline & 0.904273064 & 5.036857363 & 0.503369036 \\
\hline C & 0.726889051 & -1.180 & 0.4 \\
\hline C & 2.103654151 & -0.801575059 & 0.9434920 \\
\hline 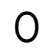 & 2.319756169 & -0.086987008 & 1.870377135 \\
\hline & 3.039460221 & -1.37 & 73017 \\
\hline & 4.386008316 & -1.16 & 0.6 \\
\hline П & 4.632738335 & -0.119613008 & 0.5821820 \\
\hline$\Pi$ & 4.985502357 & -1.731991125 & -0.0682460 \\
\hline & & & \\
\hline & & & \\
\hline & 0.52 & -3.65 & -0.2 \\
\hline C & 1.100993082 & -0.307481 & 5.2 \\
\hline U & 0.42 & 0.33 & \\
\hline & & & \\
\hline & 1.36 & -1.3 & $4 . c$ \\
\hline H & 2.010 & 13 & 5.5 \\
\hline & & & \\
\hline \multicolumn{4}{|c|}{ Coc } \\
\hline C & -1.70936 & -0.669029048 & 0.1944330 \\
\hline C & -2.243260163 & -1.821017132 & 0.79873305 \\
\hline C & & 0.1231 & \\
\hline C & -3.55 & -2.17 & 0 \\
\hline C & -3.886380282 & -0.250509017 & -0.8024230 \\
\hline C & -4.390278316 & -1.400781101 & -0.2005290 \\
\hline C & -0.007095002 & 1.203291088 & 0.4669950 \\
\hline C & & 2.01 & \\
\hline C & & 1.775 & -0.5273 \\
\hline C & -0.289415023 & 3.442497245 & 1.427464 \\
\hline C & 1.230139091 & 3.206248232 & -0.4533250 \\
\hline C & 0.66 & 3.989958 & 0.45 \\
\hline C & 07252021 & $-0.26741 c$ & \\
\hline $\mathrm{H}$ & -1.630922117 & -2.418494172 & \\
\hline & & 1.0170 & \\
\hline
\end{tabular}




$\begin{array}{rrrr}\mathrm{H} & -3.941523282 & -3.051118218 & 1.096674080 \\ \mathrm{H} & -4.518666326 & 0.353923026 & -1.426148101 \\ \mathrm{H} & -5.413925390 & -1.690098120 & -0.352485024 \\ \mathrm{H} & -1.235222087 & 1.610895118 & 2.151774154 \\ \mathrm{H} & 1.283781090 & 1.172107085 & -1.326825098 \\ \mathrm{H} & -0.760378054 & 4.081472296 & 2.146109153 \\ \mathrm{H} & 1.935319137 & 3.602078260 & -1.161317082 \\ \mathrm{H} & 0.905140063 & 5.037400363 & 0.502629035 \\ \mathrm{C} & 0.726752052 & -1.179726084 & 0.488402036 \\ \mathrm{C} & 2.103730151 & -0.801283059 & 0.943311068 \\ \mathrm{O} & 2.320006168 & -0.086942007 & 1.870360132 \\ \mathrm{O} & 3.039484219 & -1.377523099 & 0.227851018 \\ \mathrm{C} & 4.386035314 & -1.169780086 & 0.628960044 \\ \mathrm{H} & 4.632874334 & -0.119496007 & 0.582439040 \\ \mathrm{H} & 4.985522360 & -1.731705125 & -0.068537005 \\ \mathrm{H} & 4.536324325 & -1.533185111 & 1.634787117 \\ \mathrm{C} & 0.586089040 & -2.561372183 & 0.101780005 \\ \mathrm{~N} & 0.526373038 & -3.653115262 & -0.204525017 \\ \mathrm{C} & 1.099651078 & -0.308145024 & 5.231694378 \\ \mathrm{O} & 0.427300029 & 0.339021024 & 4.193722300 \\ \mathrm{H} & 1.000771070 & 0.384282025 & 3.438326249 \\ \mathrm{H} & 1.361230097 & -1.332517098 & 4.973892356 \\ \mathrm{H} & 2.008436145 & 0.215684016 & 5.520086395 \\ \mathrm{H} & 0.438391034 & -0.334299022 & 6.087091441\end{array}$

\section{Coord_8}

$\begin{array}{lrrr}\text { C } & -1.709268121 & -0.669028048 & 0.194411016 \\ \text { C } & -2.242869160 & -1.820540132 & 0.799380058 \\ \text { C } & -2.561579186 & 0.122678008 & -0.596955044 \\ \text { C } & -3.554996254 & -2.174666155 & 0.609550044 \\ \text { C } & -3.886232281 & -0.251165017 & -0.802423060 \\ \text { C } & -4.389959317 & -1.401088103 & -0.199922016 \\ \text { C } & -0.007067998 & 1.203364088 & 0.467547033 \\ \text { C } & -0.578581041 & 2.018817148 & 1.407191099 \\ \text { C } & 0.901103065 & 1.775946127 & -0.527740035 \\ \text { C } & -0.288689023 & 3.443064248 & 1.427916103 \\ \text { C } & 1.230178086 & 3.207022233 & -0.453719034 \\ \text { C } & 0.668470047 & 3.990525285 & 0.455791033 \\ \text { C } & -0.306724024 & -0.267643020 & 0.416129029 \\ \text { H } & -1.630381118 & -2.417518173 & 1.446838106 \\ \text { H } & -2.186566156 & 1.016491074 & -1.058339078 \\ \text { H } & -3.941093283 & -3.050708222 & 1.097992081 \\ \text { H } & -4.518560327 & 0.352879028 & -1.426481102 \\ \text { H } & -5.413603391 & -1.690531124 & -0.351675023 \\ \text { H } & -1.234108090 & 1.610739117 & 2.152938153 \\ \text { H } & 1.282987092 & 1.172091083 & -1.327342094 \\ \text { H } & -0.759594057 & 4.082115294 & 2.146476153\end{array}$




$\begin{array}{lrrr}\mathrm{H} & 1.935146139 & 3.602624259 & -1.162029084 \\ \mathrm{H} & 0.906005067 & 5.037942362 & 0.501888039 \\ \mathrm{C} & 0.726615053 & -1.179218084 & 0.488326035 \\ \mathrm{C} & 2.103806152 & -0.800991059 & 0.943132068 \\ \mathrm{O} & 2.320256168 & -0.086897006 & 1.870343135 \\ \mathrm{O} & 3.039508217 & -1.377113098 & 0.227530019 \\ \mathrm{C} & 4.386062318 & -1.169658084 & 0.628804047 \\ \mathrm{H} & 4.633011332 & -0.119379011 & 0.582696040 \\ \mathrm{H} & 4.985543358 & -1.731419126 & -0.068826005 \\ \mathrm{H} & 4.536191327 & -1.533417108 & 1.634531117 \\ \mathrm{C} & 0.585950041 & -2.561026185 & 0.101695008 \\ \mathrm{~N} & 0.526268039 & -3.652696265 & -0.204729015 \\ \mathrm{C} & 1.098308079 & -0.308810020 & 5.231239375 \\ \mathrm{O} & 0.426468030 & 0.338266026 & 4.192874299 \\ \mathrm{H} & 1.000377071 & 0.383547030 & 3.437801247 \\ \mathrm{H} & 1.360346095 & -1.333076094 & 4.973492356 \\ \mathrm{H} & 2.006744143 & 0.215265013 & 5.520285397 \\ \mathrm{H} & 0.436471031 & -0.335234025 & 6.086181436\end{array}$

\section{Coord_9}

\begin{tabular}{|c|c|c|c|}
\hline & -1.709170123 & -0.669027048 & 0.194388013 \\
\hline & -2.242476161 & -1.820063131 & 800026056 \\
\hline & -2.561382184 & 0.122229006 & -0.597117041 \\
\hline & -3.554695258 & -2.174455156 & 0.6104340 \\
\hline & -3.886084281 & -0.251819017 & -0.8024230 \\
\hline & -4.389639318 & -1.401394099 & -0.1993 \\
\hline & -0.007041000 & 1.203437088 & 0.4680990 \\
\hline & -0.577900043 & 2.018576144 & 1.407929100 \\
\hline & 0.900708066 & 1.775998129 & -0.528164039 \\
\hline & -0.287963019 & 3.443630246 & 1.428367105 \\
\hline & 1.230217087 & 3.207796229 & -0.454112 \\
\hline & 0.669227051 & 3.991092288 & 0.4552590 \\
\hline & -0.306195022 & -0.267867020 & 0.4162420 \\
\hline & 9119 & -2.41 & \\
\hline & -2.186535156 & 1.015930072 & -1.058855079 \\
\hline & -3.940661284 & -3.050298220 & 1.099311081 \\
\hline & -4.518454327 & 0.351835025 & -1.426811102 \\
\hline & -5.413281392 & -1.690963123 & -0.3 \\
\hline & -1.232994087 & 1.610583115 & 2.154102153 \\
\hline & 1.282193093 & 1.172075086 & -1.327857094 \\
\hline & -0.758813055 & 4.082758292 & 2.146841153 \\
\hline & 1.934972140 & 3.603170259 & -1.162741082 \\
\hline & 8065 & 5.0384 & 0. \\
\hline & 0.726479055 & -1.178709085 & 0.488 \\
\hline & 2.103883152 & -0.800698058 & 0.942952069 \\
\hline & 2.320507167 & -0.086852004 & 1.870326133 \\
\hline & & & \\
\hline
\end{tabular}




$\begin{array}{lrrr}\mathrm{C} & 4.386089317 & -1.169536083 & 0.628648046 \\ \mathrm{H} & 4.633148331 & -0.119263010 & 0.582954040 \\ \mathrm{H} & 4.985563360 & -1.731134122 & -0.069116005 \\ \mathrm{H} & 4.536057329 & -1.533649110 & 1.634275116 \\ \mathrm{C} & 0.585810042 & -2.560679183 & 0.101611007 \\ \mathrm{~N} & 0.526163040 & -3.652277262 & -0.204933013 \\ \mathrm{C} & 1.096964080 & -0.309474021 & 5.230784378 \\ \mathrm{O} & 0.425636031 & 0.337511022 & 4.192025303 \\ \mathrm{H} & 0.999983072 & 0.382813029 & 3.437276245 \\ \mathrm{H} & 1.359461099 & -1.333635096 & 4.973091357 \\ \mathrm{H} & 2.005052147 & 0.214845016 & 5.520482400 \\ \mathrm{H} & 0.434551033 & -0.336168023 & 6.085271436\end{array}$

$\begin{array}{lrrr}\text { Coord_10 } & & & \\ \text { C } & -1.708031122 & -0.629241047 & 0.206835013 \\ \text { C } & -2.238540162 & -1.777398126 & 0.819850057 \\ \text { C } & -2.565946184 & 0.164201012 & -0.575851042 \\ \text { C } & -3.554183258 & -2.127292155 & 0.645348049 \\ \text { C } & -3.894279279 & -0.205253017 & -0.765988053 \\ \text { C } & -4.395187318 & -1.352188098 & -0.155895009 \\ \text { C } & 0.003921002 & 1.237125088 & 0.460846035 \\ \text { C } & -0.552764039 & 2.054699149 & 1.406560102 \\ \text { C } & 0.902225063 & 1.805609131 & -0.546826041 \\ \text { C } & -0.257279020 & 3.479484248 & 1.422469102 \\ \text { C } & 1.238294088 & 3.236939232 & -0.477438034 \\ \text { C } & 0.691080050 & 4.022721292 & 0.437407029 \\ \text { C } & -0.300763022 & -0.233388016 & 0.412924030 \\ \text { H } & -1.620805116 & -2.375219173 & 1.461532104 \\ \text { H } & -2.193109157 & 1.056078078 & -1.042718078 \\ \text { H } & -3.937808283 & -3.001162218 & 1.139544084 \\ \text { H } & -4.531352324 & 0.399944031 & -1.384069101 \\ \text { H } & -5.421516390 & -1.638170120 & -0.295644021 \\ \text { H } & -1.200595088 & 1.649734120 & 2.160680154 \\ \text { H } & 1.272147091 & 1.199667086 & -1.350364098 \\ \text { H } & -0.717676054 & 4.120919296 & 2.145590157 \\ \text { H } & 1.936374137 & 3.628982261 & -1.194454087 \\ \text { H } & 0.933213069 & 5.069225363 & 0.479691032 \\ \text { C } & 0.728590054 & -1.147286084 & 0.473917036 \\ \text { C } & 2.112570153 & -0.774322055 & 0.912902066 \\ \text { O } & 2.342319170 & -0.060913006 & 1.837461130 \\ \text { O } & 3.038002221 & -1.354171100 & 0.187073016 \\ \text { C } & 4.389703317 & -1.152041081 & 0.573535040 \\ \text { H } & 4.640256335 & -0.102732005 & 0.524761037 \\ \text { H } & 4.979283360 & -1.716173122 & -0.130582011 \\ \text { H } & 4.549326327 & -1.516387110 & 1.577594116 \\ \text { C } & 0.578490044 & -2.529145182 & 0.089841004 \\ \text { N } & 0.511414039 & -3.620645258 & -0.215361017\end{array}$




$\begin{array}{rrrr}\mathrm{C} & 1.153798081 & -0.277420019 & 5.211047374 \\ \mathrm{O} & 0.473879036 & 0.371271028 & 4.178956303 \\ \mathrm{H} & 1.040404075 & 0.413945029 & 3.418157246 \\ \mathrm{H} & 1.410062102 & -1.302621092 & 4.951234357 \\ \mathrm{H} & 2.066652146 & 0.243953019 & 5.490916394 \\ \mathrm{H} & 0.500249034 & -0.301340021 & 6.072415434\end{array}$

LIIC path connecting the $T_{2}\left({ }^{3} \pi \pi^{*}\right) / T_{1}\left({ }^{3} \pi \pi^{*}\right)$ conical intersection and $T_{1}\left({ }^{3} \pi \pi^{*}\right)$ minimum Coord_1

$\begin{array}{lrrr}\mathrm{C} & -1.708031122 & -0.629241047 & 0.206835013 \\ \mathrm{C} & -2.238540162 & -1.777398126 & 0.819850057 \\ \mathrm{C} & -2.565946184 & 0.164201012 & -0.575851042 \\ \mathrm{C} & -3.554183258 & -2.127292155 & 0.645348049 \\ \mathrm{C} & -3.894279279 & -0.205253017 & -0.765988053 \\ \mathrm{C} & -4.395187318 & -1.352188098 & -0.155895009 \\ \mathrm{C} & 0.003921002 & 1.237125088 & 0.460846035 \\ \mathrm{C} & -0.552764039 & 2.054699149 & 1.406560102 \\ \mathrm{C} & 0.902225063 & 1.805609131 & -0.546826041 \\ \mathrm{C} & -0.257279020 & 3.479484248 & 1.422469102 \\ \mathrm{C} & 1.238294088 & 3.236939232 & -0.477438034 \\ \mathrm{C} & 0.691080050 & 4.022721292 & 0.437407029 \\ \mathrm{C} & -0.300763022 & -0.233388016 & 0.412924030 \\ \mathrm{H} & -1.620805116 & -2.375219173 & 1.461532104 \\ \mathrm{H} & -2.193109157 & 1.056078078 & -1.042718078 \\ \mathrm{H} & -3.937808283 & -3.001162218 & 1.139544084 \\ \mathrm{H} & -4.531352324 & 0.399944031 & -1.384069101 \\ \mathrm{H} & -5.421516390 & -1.638170120 & -0.295644021 \\ \mathrm{H} & -1.200595088 & 1.649734120 & 2.160680154 \\ \mathrm{H} & 1.272147091 & 1.199667086 & -1.350364098 \\ \mathrm{H} & -0.717676054 & 4.120919296 & 2.145590157 \\ \mathrm{H} & 1.936374137 & 3.628982261 & -1.194454087 \\ \mathrm{H} & 0.933213069 & 5.069225363 & 0.479691032 \\ \mathrm{C} & 0.728590054 & -1.147286084 & 0.473917036 \\ \mathrm{C} & 2.112570153 & -0.774322055 & 0.912902066 \\ \mathrm{O} & 2.342319170 & -0.060913006 & 1.837461130 \\ \mathrm{O} & 3.038002221 & -1.354171100 & 0.187073016 \\ \mathrm{C} & 4.389703317 & -1.152041081 & 0.573535040 \\ \mathrm{H} & 4.640256335 & -0.102732005 & 0.524761037 \\ \mathrm{H} & 4.979283360 & -1.716173122 & -0.130582011 \\ \mathrm{H} & 4.549326327 & -1.516387110 & 1.577594116 \\ \mathrm{C} & 0.578490044 & -2.529145182 & 0.089841004 \\ \mathrm{~N} & 0.511414039 & -3.620645258 & -0.215361017 \\ \mathrm{C} & 1.153798081 & -0.277420019 & 5.211047374 \\ \mathrm{O} & 0.473879036 & 0.371271028 & 4.178956303 \\ \mathrm{H} & 1.040404075 & 0.413945029 & 3.418157246 \\ \mathrm{H} & 1.410062102 & -1.302621092 & 4.951234357 \\ \mathrm{H} & 2.066652146 & 0.243953019 & 5.490916394 \\ & & & \\ & & & \end{array}$


$\begin{array}{llll}H & 0.500249034 & -0.301340021 & 6.072415434\end{array}$

Coord_2

\begin{tabular}{|c|c|c|c|}
\hline & -1.706642121 & -0.624971047 & 0.217043017 \\
\hline & -2.239504159 & -1.782628127 & 0.823147059 \\
\hline & -2.569532186 & 0.170109012 & -0.568231043 \\
\hline & -3.551244255 & -2.132399154 & 0.646146048 \\
\hline & -3.895520279 & 1633016 & 5 \\
\hline & -4.394663316 & -1.354154097 & -0.1544880 \\
\hline & 0.008167999 & 1.241166092 & 0.444498030 \\
\hline & -0.563790040 & 2.072473149 & 1.390587099 \\
\hline & 0.913842065 & 1.808825132 & -0.534253038 \\
\hline & -0.262250021 & 3.470852250 & 1.409929104 \\
\hline & 1.239427088 & 3.222341232 & -0.476928034 \\
\hline & 0.678044049 & 4.014760291 & 0.443536034 \\
\hline & -0.312495025 & -0.214813017 & 0.409000027 \\
\hline & -1.621920119 & -2.383960171 & 1.461 \\
\hline & -2.195371157 & 1.061059075 & -1.035420072 \\
\hline & -3.935731283 & -3.008323214 & 1.136371084 \\
\hline & -4.533821327 & 0.404185027 & -1.375499097 \\
\hline & -5.419713388 & -1.642177119 & -0.297782019 \\
\hline & -1.222367086 & 1.668397122 & 2.136351156 \\
\hline & 1.304301092 & 1.198438088 & -1.326981094 \\
\hline & -0.717215050 & 4.112452296 & 2.138873156 \\
\hline & 1.93 & 3.618228262 & $-1.19230<$ \\
\hline & 0.917505067 & 5.061835365 & 0.485324034 \\
\hline & 0.737862054 & -1.154910084 & 0.466964034 \\
\hline & 2.114834153 & -0.779882056 & 0.913917065 \\
\hline & 2.337 & -0.065690005 & 1.8 \\
\hline & 3.042585219 & -1.358475099 & 0.190188013 \\
\hline & 4.392581316 & -1.155650085 & 0.581566040 \\
\hline & 4.642312332 & -0.106112008 & 0.533615041 \\
\hline & 4.984055359 & -1.718259123 & -0.122291010 \\
\hline & 326 & -1.521330112 & 1.585363115 \\
\hline & 0.585781043 & -2.529345180 & 0.075521007 \\
\hline & 0.514286037 & -3.621669259 & -0.230950017 \\
\hline & 1.149903083 & -0.278107018 & 5.213957378 \\
\hline & 0033 & 0.369649025 & 4.181316301 \\
\hline & 1.037937072 & 0.416092029 & 3.421821248 \\
\hline & 1.407483099 & -1.303044095 & 4.954240359 \\
\hline & 2.062491146 & 0.243685017 & 5.493971397 \\
\hline & & & \\
\hline
\end{tabular}

Coord_3

$\begin{array}{lrrr}C & -1.699940123 & -0.607080046 & 0.225750015 \\ \text { C } & -2.238934161 & -1.795131130 & 0.814788059 \\ \text { C } & -2.579740184 & 0.177623011 & -0.571138041\end{array}$




\begin{tabular}{|c|c|c|c|}
\hline & 253 & -2 & 3704 \\
\hline C & -3.900857280 & -0.199414017 & \\
\hline C & -4.398476319 & -1.355141097 & -0 \\
\hline C & 19075004 & 1.273315091 & 2402 \\
\hline C & 71791041 & 2.112036155 & 36096 \\
\hline C & 22289068 & 1.826103134 & ror \\
\hline C & -0.268904017 & 3.473188250 & 1.38232 \\
\hline & 1.247239089 & 3.169403229 & -0.46163803 \\
\hline & 0.648581047 & 4.000125286 & 0.476386035 \\
\hline C & -0.355574027 & -0.169 & 0.40 \\
\hline H & -1.623815118 & -2.391071170 & 1.46 \\
\hline & -2.191693158 & 1.054318077 & -1.05202307 \\
\hline & -3.934703282 & -3.015414216 & 1.12271808 \\
\hline & -4.5 & & \\
\hline & -5.423869393 & -1.643 & -0.30 \\
\hline П & -1.241199091 & 1.685859119 & 2.09228 \\
\hline & 60098 & 1.203268084 & -1.277505090 \\
\hline & -0.743768053 & 4.11 & \\
\hline & 341142 & $3.5 \mathrm{~s}$ & -1.1 \\
\hline $\mathrm{H}$ & 0.906205064 & 5.046946366 & 0.48577 \\
\hline 乙 & 0.768786056 & -1.18 & 0.48305 \\
\hline  & 2.126699153 & & \\
\hline & 2.33 & & \\
\hline 0 & 3.056 & -1.3 & 0.20 \\
\hline$C$ & 4.400933315 & -1.15 & 0.60 \\
\hline П & 4.652640337 & -0.1 & 0. \\
\hline 11 & & & \\
\hline & 4.54 & -1.5 & 1.6 \\
\hline C & 0.613499045 & -2.53 & 0.05 \\
\hline $\mathrm{N}$ & 0.517785037 & -3.62 & -0.24391 \\
\hline 乙 & & & \\
\hline 0 & 0.457 & 0.3 & \\
\hline H & 1.029087075 & 0.408 & 3.43062924 \\
\hline $\mathrm{H}$ & 1.395001099 & -1.306 & 4.971060 \\
\hline $\mathrm{H}$ & & & \\
\hline & & -0.304 & 6.0825 \\
\hline \multicolumn{4}{|c|}{ Coc } \\
\hline 0 & -1.7 & & \\
\hline C & -2.112937153 & -1.91 & 0.737203054 \\
\hline C & -2.743257198 & 0.23 & -0.2 \\
\hline C & -3.404390244 & -2.338622169 & 0.6284380 \\
\hline C & -4.049632294 & -0.222655016 & -0.34810402 \\
\hline C & -4.393139318 & -1.509671110 & 0.06795 \\
\hline 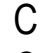 & & & 0.2 \\
\hline C & -0.6 & 2.18 & 1.1416 \\
\hline & & 1.736957125 & $-0.55 \varepsilon$ \\
\hline
\end{tabular}

S65 


$\begin{array}{rrrr}\text { C } & -0.256556021 & 3.534509252 & 1.134695079 \\ \text { C } & 1.378606097 & 3.064814219 & -0.566850039 \\ \text { C } & 0.742293051 & 3.976716289 & 0.274847022 \\ \text { C } & -0.367515028 & -0.155019009 & 0.350699024 \\ \text { H } & -1.386687100 & -2.585295185 & 1.170710084 \\ \text { H } & -2.490643177 & 1.214541089 & -0.570262041 \\ \text { H } & -3.670951266 & -3.322130239 & 0.977831072 \\ \text { H } & -4.789507344 & 0.419704028 & -0.789699056 \\ \text { H } & -5.399898388 & -1.866609133 & -0.048341003 \\ \text { H } & -1.376588101 & 1.848788132 & 1.824304132 \\ \text { H } & 1.521696107 & 1.054869077 & -1.224725089 \\ \text { H } & -0.745950052 & 4.217593301 & 1.800283131 \\ \text { H } & 2.142924156 & 3.411572243 & -1.237072091 \\ \text { H } & 1.027179073 & 5.011643358 & 0.254601018 \\ \text { C } & 0.773936057 & -1.155500085 & 0.320646022 \\ \text { C } & 2.045088147 & -0.907574066 & 1.072631076 \\ \text { O } & 2.094093151 & -0.324111021 & 2.106598149 \\ \text { O } & 3.079698224 & -1.381457098 & 0.420411030 \\ \text { C } & 4.372842317 & -1.149887081 & 0.959656067 \\ \text { H } & 4.573457328 & -0.089433008 & 1.001284071 \\ \text { H } & 5.056730366 & -1.641755116 & 0.288309021 \\ \text { H } & 4.439371322 & -1.572548114 & 1.949580141 \\ \text { C } & 0.744834055 & -2.320071167 & -0.511820039 \\ \text { N } & 0.698060051 & -3.301874239 & -1.074714076 \\ \text { C } & 1.067916075 & -0.281762019 & 5.326864382 \\ \text { O } & 0.285607021 & 0.395673026 & 4.390497316 \\ \text { H } & 0.753313056 & 0.412881029 & 3.566519258 \\ \text { H } & 1.308616093 & -1.293699094 & 5.007464361 \\ \text { H } & 1.999561144 & 0.240456020 & 5.537427396 \\ \text { H } & 0.500375036 & -0.345415025 & 6.245451447\end{array}$

\section{Coord_5}

$\begin{array}{lrrr}\text { C } & -1.714538123 & -0.628060045 & 0.338664025 \\ \text { C } & -2.053779148 & -1.958435139 & 0.720157054 \\ \text { C } & -2.776248198 & 0.202029015 & -0.122448006 \\ \text { C } & -3.335175241 & -2.412664173 & 0.644827048 \\ \text { C } & -4.076552292 & -0.283090021 & -0.214184015 \\ \text { C } & -4.367578316 & -1.591800117 & 0.163885014 \\ \text { C } & 0.007762998 & 1.267382094 & 0.232237019 \\ \text { C } & -0.626376045 & 2.203969160 & 1.065377077 \\ \text { C } & 1.046844078 & 1.720242125 & -0.598648046 \\ \text { C } & -0.247284015 & 3.545477255 & 1.046782074 \\ \text { C } & 1.415167102 & 3.044284222 & -0.615684045 \\ \text { C } & 0.772869056 & 3.972506287 & 0.203651012 \\ \text { C } & -0.367927024 & -0.156324013 & 0.310820020 \\ \text { H } & -1.290602095 & -2.620311188 & 1.086402078 \\ \text { H } & -2.560863183 & 1.192462084 & -0.467031033\end{array}$




$\begin{array}{rrrr}\mathrm{H} & -3.558104255 & -3.419245245 & 0.949317071 \\ \mathrm{H} & -4.851257349 & 0.351575024 & -0.603994043 \\ \mathrm{H} & -5.365279385 & -1.976930144 & 0.067215004 \\ \mathrm{H} & -1.387646101 & 1.876005136 & 1.747585126 \\ \mathrm{H} & 1.554668109 & 1.029150073 & -1.250175091 \\ \mathrm{H} & -0.734909055 & 4.244018307 & 1.702617125 \\ \mathrm{H} & 2.203909157 & 3.367683240 & -1.270691092 \\ \mathrm{H} & 1.071414079 & 5.004839361 & 0.188468012 \\ \mathrm{C} & 0.783025057 & -1.144035084 & 0.260260018 \\ \mathrm{C} & 1.993583145 & -0.954394066 & 1.114358078 \\ \mathrm{O} & 1.968311143 & -0.449784035 & 2.185560158 \\ \mathrm{O} & 3.072352222 & -1.371259101 & 0.498028035 \\ \mathrm{C} & 4.331935312 & -1.134386082 & 1.114603081 \\ \mathrm{H} & 4.500070323 & -0.070992007 & 1.203971086 \\ \mathrm{H} & 5.059465366 & -1.577294115 & 0.454488034 \\ \mathrm{H} & 4.369632317 & -1.596908117 & 2.088384149 \\ \mathrm{C} & 0.822624057 & -2.188294157 & -0.718048049 \\ \mathrm{~N} & 0.817885058 & -3.075814221 & -1.434488104 \\ \mathrm{C} & 1.046210076 & -0.273089021 & 5.374851387 \\ \mathrm{O} & 0.236538018 & 0.402411029 & 4.460965320 \\ \mathrm{H} & 0.659465048 & 0.375307026 & 3.613108261 \\ \mathrm{H} & 1.244912090 & -1.297196093 & 5.065935362 \\ \mathrm{H} & 1.999992143 & 0.229012017 & 5.526073395 \\ \mathrm{H} & 0.521807038 & -0.299658021 & 6.320498454\end{array}$

\section{Coord_6}

$\begin{array}{lrrr}\text { C } & -1.708199125 & -0.636824046 & 0.337384025 \\ \text { C } & -2.016133145 & -1.976444142 & 0.706462052 \\ \text { C } & -2.790243200 & 0.183448014 & -0.083651006 \\ \text { C } & -3.294332235 & -2.448247179 & 0.664361049 \\ \text { C } & -4.087391292 & -0.316998022 & -0.140554012 \\ \text { C } & -4.350268314 & -1.632880115 & 0.230527014 \\ \text { C } & 0.006211001 & 1.273450090 & 0.201083015 \\ \text { C } & -0.627586045 & 2.212475161 & 1.030569073 \\ \text { C } & 1.048113077 & 1.722717124 & -0.630179046 \\ \text { C } & -0.247859019 & 3.553864254 & 1.007598071 \\ \text { C } & 1.419607100 & 3.045058217 & -0.648447049 \\ \text { C } & 0.776269056 & 3.976259286 & 0.166780010 \\ \text { C } & -0.363631026 & -0.151015010 & 0.280145023 \\ \text { H } & -1.231696089 & -2.633657192 & 1.034825076 \\ H & -2.597410185 & 1.181601086 & -0.422817030 \\ \text { H } & -3.494970250 & -3.462605250 & 0.958428069 \\ H & -4.881243353 & 0.313179024 & -0.497265035 \\ H & -5.346794382 & -2.028081147 & 0.163991013 \\ H & -1.392470102 & 1.888792138 & 1.711072123 \\ H & 1.554458111 & 1.029017075 & -1.280040093 \\ H & -0.740258052 & 4.256066307 & 1.655830120\end{array}$




$\begin{array}{rrrr}\mathrm{H} & 2.208680161 & 3.365900241 & -1.303941092 \\ \mathrm{H} & 1.075951076 & 5.008079360 & 0.147457013 \\ \mathrm{C} & 0.789004056 & -1.131131081 & 0.198973016 \\ \mathrm{C} & 1.963091143 & -0.996130069 & 1.109914079 \\ \mathrm{O} & 1.897267139 & -0.536344039 & 2.202977159 \\ \mathrm{O} & 3.066878222 & -1.388771100 & 0.521905036 \\ \mathrm{C} & 4.299408311 & -1.159519085 & 1.193516088 \\ \mathrm{H} & 4.456352318 & -0.097927007 & 1.318241097 \\ \mathrm{H} & 5.057067362 & -1.580150116 & 0.553033041 \\ \mathrm{H} & 4.301545308 & -1.646156119 & 2.156780155 \\ \mathrm{C} & 0.882278066 & -2.073711148 & -0.873448061 \\ \mathrm{~N} & 0.912845063 & -2.883465208 & -1.671226119 \\ \mathrm{C} & 1.034673075 & -0.282176021 & 5.408110388 \\ \mathrm{O} & 0.219358014 & 0.388356029 & 4.495662324 \\ \mathrm{H} & 0.616602046 & 0.321510025 & 3.637542264 \\ \mathrm{H} & 1.201904088 & -1.318260094 & 5.122331369 \\ \mathrm{H} & 2.002958145 & 0.201064013 & 5.523723399 \\ \mathrm{H} & 0.533822039 & -0.271676017 & 6.366916459\end{array}$

\section{Coord_7}

$\begin{array}{lrrr}\text { C } & -1.696901122 & -0.642206049 & 0.333882025 \\ \text { C } & -1.972405144 & -1.988289145 & 0.694441050 \\ \text { C } & -2.800629203 & 0.168466011 & -0.039583003 \\ \text { C } & -3.247499234 & -2.477006179 & 0.691256049 \\ \text { C } & -4.092657294 & -0.346502024 & -0.057545004 \\ \text { C } & -4.326069313 & -1.669804119 & 0.305664024 \\ \text { C } & 0.000272002 & 1.287357090 & 0.160436011 \\ \text { C } & -0.630866045 & 2.224625160 & 0.995132073 \\ \text { C } & 1.045788073 & 1.736977123 & -0.665374048 \\ \text { C } & -0.245856020 & 3.564058255 & 0.979645071 \\ \text { C } & 1.421561103 & 3.058452223 & -0.677139050 \\ \text { C } & 0.780175057 & 3.987440288 & 0.141255008 \\ \text { C } & -0.355704025 & -0.140174010 & 0.237678019 \\ \text { H } & -1.167522082 & -2.638684190 & 0.986640069 \\ \text { H } & -2.633494189 & 1.174826082 & -0.367879028 \\ \text { H } & -3.425755247 & -3.496900249 & 0.980613068 \\ \text { H } & -4.906562351 & 0.278692018 & -0.376623026 \\ \text { H } & -5.319863383 & -2.075040151 & 0.269572019 \\ \text { H } & -1.393038100 & 1.900489135 & 1.678179122 \\ \text { H } & 1.553536109 & 1.044397078 & -1.314752094 \\ \text { H } & -0.734596052 & 4.264594306 & 1.632416117 \\ \text { H } & 2.215036161 & 3.378612243 & -1.327448093 \\ \text { H } & 1.083639079 & 5.018229361 & 0.128496010 \\ \text { C } & 0.800339059 & -1.100433080 & 0.122117011 \\ \text { C } & 1.932526140 & -1.033776072 & 1.090533079 \\ \text { O } & 1.820846134 & -0.632665046 & 2.204588160 \\ \text { O } & 3.060176219 & -1.404650101 & 0.533458039 \\ & & & \end{array}$




$\begin{array}{lrrr}\mathrm{C} & 4.262833304 & -1.208706090 & 1.266458092 \\ \mathrm{H} & 4.414260316 & -0.153911012 & 1.447443104 \\ \mathrm{H} & 5.047099361 & -1.599427117 & 0.638673044 \\ \mathrm{H} & 4.226194305 & -1.740159124 & 2.205365156 \\ \mathrm{C} & 0.942146068 & -1.939805142 & -1.030681073 \\ \mathrm{~N} & 1.005693075 & -2.662396194 & -1.907715135 \\ \mathrm{C} & 1.034642076 & -0.290908022 & 5.440060391 \\ \mathrm{O} & 0.207740018 & 0.359168024 & 4.523239328 \\ \mathrm{H} & 0.579883039 & 0.250628018 & 3.657697265 \\ \mathrm{H} & 1.181790087 & -1.338569097 & 5.186777374 \\ \mathrm{H} & 2.011752144 & 0.182526013 & 5.517568395 \\ \mathrm{H} & 0.555136039 & -0.240251017 & 6.408895461\end{array}$

Coord_8

$\begin{array}{lrrr}\text { C } & -1.667091117 & -0.642998047 & 0.300988023 \\ \text { C } & -1.889511136 & -1.996704143 & 0.661688048 \\ \text { C } & -2.804208204 & 0.143179012 & -0.010152001 \\ \text { C } & -3.152005225 & -2.518537179 & 0.718231049 \\ \text { C } & -4.083993291 & -0.400502028 & 0.041347005 \\ \text { C } & -4.267123307 & -1.732136124 & 0.402893030 \\ \text { C } & 0.000540999 & 1.321482096 & 0.109088006 \\ \text { C } & -0.636355047 & 2.246955159 & 0.952444069 \\ \text { C } & 1.039343075 & 1.787265128 & -0.717648050 \\ \text { C } & -0.257645020 & 3.589085259 & 0.949264068 \\ \text { C } & 1.410366104 & 3.109303225 & -0.714773051 \\ \text { C } & 0.766361054 & 4.026030290 & 0.116195010 \\ \text { C } & -0.336609024 & -0.112103009 & 0.159576014 \\ \text { H } & -1.053180076 & -2.628583191 & 0.902470067 \\ \text { H } & -2.677596192 & 1.157443081 & -0.333458022 \\ \text { H } & -3.290591238 & -3.545975253 & 1.003370071 \\ \text { H } & -4.929626357 & 0.210386015 & -0.218217018 \\ \text { H } & -5.252411376 & -2.158864155 & 0.422778030 \\ \text { H } & -1.398571103 & 1.914333137 & 1.631039118 \\ \text { H } & 1.546233109 & 1.106164080 & -1.378988100 \\ \text { H } & -0.749875055 & 4.280928309 & 1.608546116 \\ \text { H } & 2.201367159 & 3.438824247 & -1.363373100 \\ \text { H } & 1.066895075 & 5.057743365 & 0.115178011 \\ \text { C } & 0.828249058 & -1.043232073 & 0.015614999 \\ \text { C } & 1.899324136 & -1.057895077 & 1.048996078 \\ \text { O } & 1.720327121 & -0.744014052 & 2.182797159 \\ \text { O } & 3.058039221 & -1.400810099 & 0.540291040 \\ \text { C } & 4.209162301 & -1.265789090 & 1.363148100 \\ \text { H } & 4.344931313 & -0.228930016 & 1.637717118 \\ \text { H } & 5.034766362 & -1.607729114 & 0.760933055 \\ \text { H } & 4.110930297 & -1.869376132 & 2.253067162 \\ \text { C } & 1.054240076 & -1.761666125 & -1.203223088 \\ \text { N } & 1.205514087 & -2.376963172 & -2.147223155\end{array}$




$\begin{array}{rrrr}\mathrm{C} & 1.038024074 & -0.306987020 & 5.470551393 \\ \mathrm{O} & 0.200166015 & 0.306390023 & 4.538804325 \\ \mathrm{H} & 0.533062040 & 0.122752008 & 3.669840264 \\ \mathrm{H} & 1.140372082 & -1.374888098 & 5.288783379 \\ \mathrm{H} & 2.032880144 & 0.135479012 & 5.480524395 \\ \mathrm{H} & 0.596848044 & -0.171792012 & 6.449618465\end{array}$

\section{Coord_9}

\begin{tabular}{|c|c|c|c|}
\hline & -1.583200114 & -0.644520045 & 0.247945016 \\
\hline & -1.732471125 & -2.010773145 & 0.593460045 \\
\hline & -2.764112200 & 0.101196005 & 0.014781000 \\
\hline & -2.970144213 & -2.580449187 & 0.717943050 \\
\hline & -4.018093288 & -0.489397037 & 0.136359007 \\
\hline & -4.129640296 & -1.830895130 & 0.490085036 \\
\hline & -0.001294002 & 1.392707099 & 0.060111006 \\
\hline & -0.648604045 & 2.266081165 & 0.950584069 \\
\hline & 0.995715073 & 1.923622140 & -0.778090056 \\
\hline & -0.322971025 & 3.621960258 & 0.978322070 \\
\hline & 1.317727096 & 3.258265234 & -0.7415020 \\
\hline & 0.660564049 & 4.123884297 & 0.132889007 \\
\hline & -0.279338022 & -0.055595002 & 0.070717004 \\
\hline & -0.860996060 & -2.612180189 & 0.779963058 \\
\hline & -2.694454196 & 1.124654078 & -0.296224021 \\
\hline & -3.054371218 & -3.615335261 & 0.995830073 \\
\hline & -4.900335353 & 0.093985007 & -0.057315003 \\
\hline & -5.095564367 & -2.292074166 & 0.575878041 \\
\hline & -1.375307100 & 1.882785135 & 1.641739119 \\
\hline & 1.508567108 & 1.284004091 & -1.474825105 \\
\hline & -0.825088060 & 4.273997309 & 1.670121118 \\
\hline & 2.080300151 & 3.636023260 & -1.398370100 \\
\hline & 0.920002064 & 5.166469371 & 0.156337010 \\
\hline & 0.919106066 & -0.929939066 & -0.090637008 \\
\hline & 1.920740140 & -1.024625074 & 1.003635073 \\
\hline & 1.664629120 & -0.789040056 & 2.143109157 \\
\hline & 3.107140223 & -1.357 & 0.556027041 \\
\hline & 4.182041300 & -1.372020100 & 1.487559105 \\
\hline & 4.318938311 & -0.388183030 & 1.915518140 \\
\hline & 5.051009362 & -1.663976121 & 0.920107069 \\
\hline & 3.985017286 & -2.088042152 & 2.274950165 \\
\hline & 1.212819088 & -1.564140112 & -1.337435097 \\
\hline & 1.414917102 & -2.102250154 & -2.318579168 \\
\hline & 1.053384074 & -0.341923027 & 5.469401396 \\
\hline & 0.195336013 & 0.251012016 & 4.543400325 \\
\hline & 0.493363036 & 0.027974003 & 3.671218262 \\
\hline & 1.080171079 & -1.425890101 & 5.369050387 \\
\hline & 2.071468151 & 0.035524002 & 5.384959386 \\
\hline & 0.68 & & \\
\hline
\end{tabular}




\begin{tabular}{lrrr} 
Coord_10 & & & \\
C & -1.483897108 & -0.613726047 & 0.254237018 \\
C & -1.567900112 & -1.998227141 & 0.544338040 \\
C & -2.699690194 & 0.087816007 & 0.074916004 \\
C & -2.776670201 & -2.626607191 & 0.669582049 \\
C & -3.924095284 & -0.560557042 & 0.200352015 \\
C & -3.971391284 & -1.918546139 & 0.501450039 \\
C & -0.001006998 & 1.501887108 & 0.122736011 \\
C & -0.665273049 & 2.315394166 & 1.054999075 \\
C & 0.936720070 & 2.106460150 & -0.731992055 \\
C & -0.413116031 & 3.686008264 & 1.106142077 \\
C & 1.190413088 & 3.455020246 & -0.669265046 \\
C & 0.515507040 & 4.261014305 & 0.246248018 \\
C & -0.209220015 & 0.040327005 & 0.090774007 \\
H & -0.668367048 & -2.569154184 & 0.686046050 \\
H & -2.680402191 & 1.125722080 & -0.191140013 \\
H & -2.809334201 & -3.675182266 & 0.903512065 \\
H & -4.834063348 & -0.007485001 & 0.053285006 \\
H & -4.914938354 & -2.422039173 & 0.595750044 \\
H & -1.349883097 & 1.876794134 & 1.755813128 \\
H & 1.455222104 & 1.513651110 & -1.463619105 \\
H & -0.928719069 & 4.294232311 & 1.826514130 \\
H & 1.911748138 & 3.888035278 & -1.337905096 \\
H & 0.719035050 & 5.314910384 & 0.291123022 \\
C & 1.025464073 & -0.771274057 & -0.096049005 \\
C & 1.970523141 & -0.967254068 & 1.032550074 \\
O & 1.667134118 & -0.792661057 & 2.170726155 \\
O & 3.162804230 & -1.326426093 & 0.623544047 \\
C & 4.181402303 & -1.461683105 & 1.603409118 \\
H & 4.329762309 & -0.523386039 & 2.117126152 \\
H & 5.069238365 & -1.741167127 & 1.060548075 \\
H & 3.916652284 & -2.228629159 & 2.316143169 \\
$\mathrm{C}$ & 1.404809103 & -1.252320090 & -1.388004100 \\
N & 1.689531122 & -1.648905121 & -2.414704173 \\
C & 1.059459076 & -0.362829026 & 5.482767392 \\
O & 0.201877014 & 0.240595019 & 4.562962330 \\
H & 0.488100034 & 0.009218003 & 3.689156265 \\
H & 1.056618077 & -1.447672105 & 5.396669386 \\
H & 2.084872148 & -0.014221004 & 5.376898387 \\
H & 0.713753051 & -0.101915008 & 6.474144465 \\
& & & \\
\hline
\end{tabular}

LIIC path connecting the $T_{1}\left({ }^{3} \pi \pi^{*}\right)$ minimum and $T_{1}\left({ }^{3} \pi \pi^{*}\right) / S_{0}$ crossing point Coord_1
C -1.483897108
$-0.613726047$
0.254237018
$\begin{array}{llll}\text { C } & -1.567900112 & -1.998227141 & 0.544338040\end{array}$
$\begin{array}{llll}\text { C } & -2.699690194 & 0.087816007 & 0.074916004\end{array}$ 


\begin{tabular}{|c|c|c|c|}
\hline & 702 & 191 & 0.669582049 \\
\hline C & -3.924095284 & -0.560557042 & \\
\hline C & -3.971391284 & -1.918546139 & 0039 \\
\hline & -0.001006998 & 1.501887108 & 0.12273601 \\
\hline & -0.665273049 & 2.315394166 & .054999075 \\
\hline & 6720070 & 2.106460150 & -0.10 \\
\hline$C$ & 3116031 & 6008264 & \\
\hline & 1.190413088 & 3.455020246 & -0.6692650 \\
\hline 乙 & 515507040 & 4.261014305 & 0.246248018 \\
\hline 乙 & 9220015 & 0.040327005 & \\
\hline$\Pi$ & -0.668367048 & -2.569154184 & 0.00004 \\
\hline & -2.680402191 & 1.125722080 & $-0.1911<$ \\
\hline & -2.809334201 & -3.675182266 & 0.9035120 \\
\hline & -4.834063348 & -0.007485001 & 0.053285006 \\
\hline & -4.914938354 & -2.42 & 0.5 \\
\hline$\Pi$ & -1.349883097 & 1.876794134 & 1.755813 \\
\hline & 1.455222104 & 1.513651110 & -1.463619105 \\
\hline & -0.928719069 & 4.294232311 & 1.826514130 \\
\hline & 1.9 & 3.88 & -1.3 \\
\hline & 0.719035050 & 10384 & 0.2911230 \\
\hline U & 1.025464073 & -0.771274057 & -0.09604900 \\
\hline & 1.97 & & \\
\hline 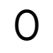 & & & \\
\hline 0 & 3.1 & -1. & 0.6 \\
\hline C & 4.181402303 & -1.461683105 & 1.603409 \\
\hline 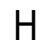 & $4.32 \mathrm{C}$ & -0.52 & 2.11 \\
\hline 11 & & & \\
\hline & 2284 & -2.22 & 2.3 \\
\hline C & 1.404809103 & -1.252320090 & -1.388004 \\
\hline $\mathrm{N}$ & 1.689531122 & -1.648905121 & -2.41470 \\
\hline 乙 & & -0.362 & 2767392 \\
\hline 0 & 0.20 & & 4.5 \\
\hline $\mathrm{H}$ & 0.488100034 & 0.009 & 3.6891562 \\
\hline$H_{2}$ & 1.056618077 & -1.447672105 & 5.3966693 \\
\hline H & & & 387 \\
\hline & & -0.10 & 6.4741 \\
\hline \multicolumn{4}{|c|}{ Coord_2 } \\
\hline C & -1.48 & -0.6 & 0.253 \\
\hline C & -1.56 & -1.998456143 & 0.541841037 \\
\hline C & -2.6 & 0.087454007 & 0.07611100 \\
\hline C & -2.773008199 & -2.627784187 & 0.667829049 \\
\hline C & -3.922275285 & -0.561818039 & 0.202490013 \\
\hline c & -3.968368285 & -1.920227140 & 0.502104038 \\
\hline 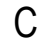 & -0.000937 & 1.50348 & 0.1231730 \\
\hline C & -0.664802 & 2.315894165 & 1.0566 \\
\hline & & $2.10 s$ & -0.73 \\
\hline
\end{tabular}




\begin{tabular}{|c|c|c|c|}
\hline & 332 & 10 & 1.107729080 \\
\hline C & 1.186716087 & 3.458354248 & \\
\hline 0 & 512174038 & 4.263260308 & $43 / 0$ \\
\hline C & -0.207878016 & 0.041699003 & 0.09026400 \\
\hline$\Gamma$ & -0.664765049 & -2.568989186 & 881947048 \\
\hline $\mathrm{H}$ & -2.680069190 & 1.125698082 & -0 \\
\hline & $-2 . \varepsilon$ & -3.676672265 & \\
\hline & -4.832769346 & -0.009077999 & 0.05 \\
\hline & -4.911474355 & -2.424374173 & 0.59735 \\
\hline$\Gamma$ & $-1.3^{2} x$ & 1.876239133 & \\
\hline & $1.452646^{1}$ & 1.517397108 & -1.400 \\
\hline $\mathrm{H}$ & -0.929513067 & 4.294232311 & 1.829045 \\
\hline & 1.906419138 & 3.892431281 & -1.34000109 \\
\hline $\mathrm{H}$ & 0.714312053 & 5.317445381 & 0.291193021 \\
\hline 0 & 1.027357073 & -0.768 & -0.0 \\
\hline$U$ & 1.970608142 & -0.968106070 & 1.0324120 \\
\hline C & 1.666156119 & -0.793365059 & 2.17031315 \\
\hline C & 3.16 & -1.3 & 2902 \\
\hline 0 & 4.17 & -1.4 & 1.60 \\
\hline$H$ & 4.3295 & -0.53 & 2.121852 \\
\hline $\mathrm{H}$ & 5.066193362 & -1.754 & 1.0652290 \\
\hline & 3.90 & & \\
\hline C & & & \\
\hline & 1.6 & -1.6 & -2.4 \\
\hline C & 1.059652078 & -0.35 & 5.480 \\
\hline 0 & 0.20 & & 4.56 \\
\hline $\mathrm{H}$ & & & \\
\hline & 1.05 & -1.4 & \\
\hline$H_{-1}$ & 2.08 & -0.0 & 5.37 \\
\hline & 9 & & \\
\hline \multicolumn{4}{|c|}{ Couldi_o } \\
\hline C & -1.480055107 & 277045 & 0.2523490 \\
\hline c & -1.5616 & 79143 & 0.539328038 \\
\hline c & & & 0.07 \\
\hline U & -2.76 & & 0 \\
\hline C & -3.92 & -0.563086038 & 0.204603012 \\
\hline C & -3.965333284 & -1.921911141 & 0.5027310 \\
\hline C & -0.000864998 & 1.505088107 & 0.1236010 \\
\hline C & -0.6 & & \\
\hline 0 & & 2.11 & -0.73 \\
\hline C & -0.41 & 3.687646263 & 1.10930308 \\
\hline C & 1.183017085 & 3.461685249 & -0.6713290 \\
\hline C & $0.508836 \mathrm{C}$ & 4.265503 & 0.24662 \\
\hline C & & & 0.08974 \\
\hline $\mathrm{H}$ & -0.6 & -2.5688 & 0.677836 \\
\hline $\mathrm{H}$ & & & \\
\hline
\end{tabular}




$\begin{array}{rrrr}\mathrm{H} & -2.799982200 & -3.678155263 & 0.897692062 \\ \mathrm{H} & -4.831461347 & -0.010681998 & 0.061610006 \\ \mathrm{H} & -4.907994353 & -2.426715173 & 0.598921043 \\ \mathrm{H} & -1.345865097 & 1.875686137 & 1.760796126 \\ \mathrm{H} & 1.450073104 & 1.521142111 & -1.467324108 \\ \mathrm{H} & -0.930311067 & 4.294230311 & 1.831558131 \\ \mathrm{H} & 1.901086137 & 3.896822282 & -1.342091094 \\ \mathrm{H} & 0.709582050 & 5.319976383 & 0.291260020 \\ \mathrm{C} & 1.029253073 & -0.766462053 & -0.098160009 \\ \mathrm{C} & 1.970689144 & -0.968961072 & 1.032262075 \\ \mathrm{O} & 1.665181121 & -0.794072055 & 2.169888156 \\ \mathrm{O} & 3.161697228 & -1.336029094 & 0.626498046 \\ \mathrm{C} & 4.175436300 & -1.481811107 & 1.610065114 \\ \mathrm{H} & 4.329331310 & -0.545918041 & 2.126559154 \\ \mathrm{H} & 5.063088362 & -1.767053128 & 1.069880079 \\ \mathrm{H} & 3.901756278 & -2.248266164 & 2.319975169 \\ \mathrm{C} & 1.408290099 & -1.245332087 & -1.391000100 \\ \mathrm{~N} & 1.692904124 & -1.638040117 & -2.419271174 \\ \mathrm{C} & 1.059887075 & -0.353557026 & 5.479088393 \\ \mathrm{O} & 0.203729013 & 0.250737018 & 4.558499329 \\ \mathrm{H} & 0.487499037 & 0.014708000 & 3.685111266 \\ \mathrm{H} & 1.048207074 & -1.438874105 & 5.399864389 \\ \mathrm{H} & 2.087584149 & -0.013710998 & 5.367232388 \\ \mathrm{H} & 0.719785053 & -0.083595008 & 6.469998467\end{array}$

\section{Coord_4}

$\begin{array}{lrrr}\text { C } & -1.478125107 & -0.613048043 & 0.251383017 \\ \text { C } & -1.558579110 & -1.998897143 & 0.536801037 \\ \text { C } & -2.695902195 & 0.086722007 & 0.078440007 \\ \text { C } & -2.765658197 & -2.630130188 & 0.664263045 \\ \text { C } & -3.918601281 & -0.564360043 & 0.206689013 \\ \text { C } & -3.962288287 & -1.923600137 & 0.503333037 \\ \text { C } & -0.000788998 & 1.506690106 & 0.124021007 \\ \text { C } & -0.663859050 & 2.316898167 & 1.059921074 \\ \text { C } & 0.930605067 & 2.115362150 & -0.734593051 \\ \text { C } & -0.416654031 & 3.688464265 & 1.110864079 \\ \text { C } & 1.179316083 & 3.465013250 & -0.672361050 \\ \text { C } & 0.505494038 & 4.267744307 & 0.246800019 \\ \text { C } & -0.205179017 & 0.044451005 & 0.089216009 \\ \text { H } & -0.657546045 & -2.568629187 & 0.673715051 \\ \text { H } & -2.679379191 & 1.125631082 & -0.183939011 \\ \text { H } & -2.795292202 & -3.679634266 & 0.894751065 \\ \text { H } & -4.830140346 & -0.012299000 & 0.065728005 \\ \text { H } & -4.904501354 & -2.429063175 & 0.600463044 \\ \text { H } & -1.343855096 & 1.875136137 & 1.763259129 \\ \text { H } & 1.447506102 & 1.524885109 & -1.469172106 \\ \text { H } & -0.931114067 & 4.294229311 & 1.834054133\end{array}$




$\begin{array}{lrrr}\mathrm{H} & 1.895751136 & 3.901209278 & -1.344176095 \\ \mathrm{H} & 0.704844050 & 5.322503385 & 0.291323019 \\ \mathrm{C} & 1.031153073 & -0.764054053 & -0.099219009 \\ \mathrm{C} & 1.970769139 & -0.969816069 & 1.032099072 \\ \mathrm{O} & 1.664210118 & -0.794782058 & 2.169451156 \\ \mathrm{O} & 3.161116229 & -1.340829097 & 0.627949045 \\ \mathrm{C} & 4.172384302 & -1.491867105 & 1.613354115 \\ \mathrm{H} & 4.329033309 & -0.557199042 & 2.131248151 \\ \mathrm{H} & 5.059925364 & -1.779986130 & 1.074502077 \\ \mathrm{H} & 3.894242279 & -2.258052165 & 2.321833169 \\ \mathrm{C} & 1.410042099 & -1.241837089 & -1.392498101 \\ \mathrm{~N} & 1.694589120 & -1.632607118 & -2.421552176 \\ \mathrm{C} & 1.060165078 & -0.348948024 & 5.477238395 \\ \mathrm{O} & 0.204719013 & 0.255791021 & 4.556265327 \\ \mathrm{H} & 0.487242037 & 0.017436999 & 3.683091264 \\ \mathrm{H} & 1.044053075 & -1.434458105 & 5.401461387 \\ \mathrm{H} & 2.088973149 & -0.013520002 & 5.362395385 \\ \mathrm{H} & 0.722906050 & -0.074477004 & 6.467890463\end{array}$

\section{Coord_5}

$\begin{array}{lrrr}\text { C } & -1.476190107 & -0.612817042 & 0.250402017 \\ \text { C } & -1.555460113 & -1.999109142 & 0.534259039 \\ \text { C } & -2.694624195 & 0.086351006 & 0.079574008 \\ \text { C } & -2.761971197 & -2.631299188 & 0.662450047 \\ \text { C } & -3.916749283 & -0.565641038 & 0.208751016 \\ \text { C } & -3.959232283 & -1.925292139 & 0.503910036 \\ \text { C } & -0.000710002 & 1.508293111 & 0.124431008 \\ \text { C } & -0.663385050 & 2.317402166 & 1.061533075 \\ \text { C } & 0.928572068 & 2.118328151 & -0.735464051 \\ \text { C } & -0.417839028 & 3.689283268 & 1.112413081 \\ \text { C } & 1.175614086 & 3.468340251 & -0.673394046 \\ \text { C } & 0.502148034 & 4.269983309 & 0.246973018 \\ \text { C } & -0.203822016 & 0.045831004 & 0.088679005 \\ \text { H } & -0.653929044 & -2.568434185 & 0.669583049 \\ \text { H } & -2.679024192 & 1.125590082 & -0.181580013 \\ \text { H } & -2.790594198 & -3.681108262 & 0.891790064 \\ \text { H } & -4.828807348 & -0.013928003 & 0.069816005 \\ \text { H } & -4.900994354 & -2.431418173 & 0.601974041 \\ \text { H } & -1.341843095 & 1.874588137 & 1.765703128 \\ \text { H } & 1.444943106 & 1.528627112 & -1.471017103 \\ \text { H } & -0.931923067 & 4.294229311 & 1.836533133 \\ \text { H } & 1.890414135 & 3.905593279 & -1.346256095 \\ \text { H } & 0.700099050 & 5.325027385 & 0.291381022 \\ \text { C } & 1.033056074 & -0.761644052 & -0.100281010 \\ \text { C } & 1.970846140 & -0.970673072 & 1.031925074 \\ \text { O } & 1.663242120 & -0.795496056 & 2.169004154 \\ \text { O } & 3.160515226 & -1.345628099 & 0.629384047\end{array}$




$\begin{array}{lrrr}\mathrm{C} & 4.169287298 & -1.501919107 & 1.616616117 \\ \mathrm{H} & 4.328681311 & -0.568490038 & 2.135918151 \\ \mathrm{H} & 5.056704362 & -1.792914126 & 1.079095076 \\ \mathrm{H} & 3.886686279 & -2.267816163 & 2.323652168 \\ \mathrm{C} & 1.411802100 & -1.238343090 & -1.393996101 \\ \mathrm{~N} & 1.696274121 & -1.627172118 & -2.423831173 \\ \mathrm{C} & 1.060484077 & -0.344357025 & 5.475382395 \\ \mathrm{O} & 0.205752015 & 0.260834017 & 4.554031326 \\ \mathrm{H} & 0.487014036 & 0.020157002 & 3.681073262 \\ \mathrm{H} & 1.039935076 & -1.430033103 & 5.403058391 \\ \mathrm{H} & 2.090384153 & -0.013371002 & 5.357558387 \\ \mathrm{H} & 0.726097052 & -0.065387004 & 6.465759467\end{array}$

Coord_6

\begin{tabular}{|c|c|c|c|}
\hline & -1.474250107 & 33045 & 7016 \\
\hline & -1.552336110 & -1.999315146 & 0.531702038 \\
\hline & -2.693340194 & 0.085977005 & 0.080687005 \\
\hline & -2.758275200 & -2.632466188 & 0.660616046 \\
\hline & -3.914887282 & -0.566928040 & 0.210786015 \\
\hline & -3.956165283 & -1.926988141 & 0.504461036 \\
\hline & -0.000626001 & 1.509898111 & 0.1248330 \\
\hline & -0.662911045 & 2.317907166 & 1.063132074 \\
\hline & 0.926542065 & 2.121293153 & -0.736337055 \\
\hline & -0.419027031 & 3.690102265 & 1.113949082 \\
\hline & 1.171910083 & 3.471664252 & -0.674427048 \\
\hline & 0.498798035 & 4.272220305 & 0.247140015 \\
\hline & -0.202460014 & 0.047214004 & 0.088133005 \\
\hline & -0.650308049 & -2.568229187 & 0.665440047 \\
\hline & -2.678661192 & 1.125544080 & -0.179240013 \\
\hline & -2.785887198 & -3.682578264 & 0.888808065 \\
\hline & -4.827461349 & -0.015569003 & 0.073875007 \\
\hline & -4.897473351 & -2.433780176 & 0.603456044 \\
\hline & -1.33 & 1.87 & 25 \\
\hline & 1.442385106 & 1.532368109 & -1.472860106 \\
\hline & -0.932736069 & 4.294230311 & 1.838996130 \\
\hline & 1.885074133 & 3.909972279 & -1.348332094 \\
\hline & 0.695346049 & 5.327547386 & 0.291436019 \\
\hline & 1.034964075 & -0.759234052 & -0.101346005 \\
\hline & 1.970920140 & -0.971533069 & 1.031739073 \\
\hline & 1.662278118 & -0.796213059 & 2.168545157 \\
\hline & 3.159896226 & -1.350425096 & 0.630802046 \\
\hline & 4.166144302 & -1.511966109 & 1.619851115 \\
\hline & 4.328275311 & -0.579791042 & 2.140570154 \\
\hline & 5.053424363 & -1.805835132 & 1.083658077 \\
\hline & 3.879087277 & -2.277559163 & 2.325433167 \\
\hline & 1.413569103 & -1.234848091 & -1.395493101 \\
\hline & & -16 & \\
\hline
\end{tabular}




$\begin{array}{rrrr}\mathrm{C} & 1.060846076 & -0.339784024 & 5.473520395 \\ \mathrm{O} & 0.206827017 & 0.265865021 & 4.551795330 \\ \mathrm{H} & 0.486814033 & 0.022867003 & 3.679057267 \\ \mathrm{H} & 1.035853077 & -1.425596100 & 5.404656390 \\ \mathrm{H} & 2.091816149 & -0.013265003 & 5.352719384 \\ \mathrm{H} & 0.729358054 & -0.056326002 & 6.463607467\end{array}$

$\begin{array}{lrrr}\text { Coord_7 } & & & \\ \text { C } & -1.472304105 & -0.612346042 & 0.248397018 \\ \text { C } & -1.549205111 & -1.999516143 & 0.529130036 \\ \text { C } & -2.692048192 & 0.085600008 & 0.081781005 \\ \text { C } & -2.754570198 & -2.633630188 & 0.658762047 \\ \text { C } & -3.913014281 & -0.568222042 & 0.212797015 \\ \text { C } & -3.953087286 & -1.928687139 & 0.504987038 \\ \text { C } & -0.000539999 & 1.511504111 & 0.125225007 \\ \text { C } & -0.662437045 & 2.318415165 & 1.064716076 \\ \text { C } & 0.924514067 & 2.124257154 & -0.737212055 \\ \text { C } & -0.420218029 & 3.690921267 & 1.115472080 \\ \text { C } & 1.168205085 & 3.474986252 & -0.675461050 \\ \text { C } & 0.495444036 & 4.274456307 & 0.247301018 \\ \text { C } & -0.201094017 & 0.048600004 & 0.087577004 \\ \text { H } & -0.646682047 & -2.568015183 & 0.661287048 \\ \text { H } & -2.678292192 & 1.125491083 & -0.176922015 \\ \text { H } & -2.781170202 & -3.684042264 & 0.885806064 \\ \text { H } & -4.826103348 & -0.017220999 & 0.077905004 \\ \text { H } & -4.893939352 & -2.436149176 & 0.604909043 \\ \text { H } & -1.337818094 & 1.873503133 & 1.770533125 \\ \text { H } & 1.439832106 & 1.536107112 & -1.474700108 \\ \text { H } & -0.933554066 & 4.294231311 & 1.841441130 \\ \text { H } & 1.879733137 & 3.914348284 & -1.350402098 \\ \text { H } & 0.690587052 & 5.330064385 & 0.291486021 \\ \text { C } & 1.036875077 & -0.756822057 & -0.102414007 \\ \text { C } & 1.970993140 & -0.972393072 & 1.031541076 \\ \text { O } & 1.661317122 & -0.796932057 & 2.168075157 \\ \text { O } & 3.159259229 & -1.355221098 & 0.632203048 \\ \text { C } & 4.162956300 & -1.522007110 & 1.623060115 \\ \text { H } & 4.327814312 & -0.591101042 & 2.145203154 \\ \text { H } & 5.050086366 & -1.818749131 & 1.088191078 \\ \text { H } & 3.871445281 & -2.287279164 & 2.327175165 \\ \mathrm{C} & 1.415344101 & -1.231354087 & -1.396991101 \\ \text { N } & 1.699642122 & -1.616301114 & -2.428383177 \\ \text { C } & 1.061250077 & -0.335230025 & 5.471653394 \\ \text { O } & 0.207946016 & 0.270885019 & 4.549559329 \\ \text { H } & 0.486642035 & 0.025566003 & 3.677044266 \\ \text { H } & 1.031806072 & -1.421149101 & 5.406255389 \\ \text { H } & 2.093270148 & -0.013200999 & 5.347879386 \\ \text { H } & 0.732689050 & -0.047295005 & 6.461432464 \\ & & & \\ & & & \end{array}$


Coord_8

$\begin{array}{lrrr}\text { C } & -1.470353108 & -0.612106044 & 0.247372017 \\ \mathrm{C} & -1.546068111 & -1.999711145 & 0.526544037 \\ \mathrm{C} & -2.690749194 & 0.085220006 & 0.082854007 \\ \mathrm{C} & -2.750857199 & -2.634791192 & 0.656889045 \\ \mathrm{C} & -3.911131283 & -0.569523040 & 0.214781017 \\ \mathrm{C} & -3.949998283 & -1.930391137 & 0.505487037 \\ \mathrm{C} & -0.000449002 & 1.513111111 & 0.125609010 \\ \mathrm{C} & -0.661961050 & 2.318924165 & 1.066286076 \\ \mathrm{C} & 0.922489065 & 2.127221155 & -0.738089055 \\ \mathrm{C} & -0.421411033 & 3.691740264 & 1.116982082 \\ \mathrm{C} & 1.164498082 & 3.478306252 & -0.676496046 \\ \mathrm{C} & 0.492085035 & 4.276689308 & 0.247456019 \\ \mathrm{C} & -0.199722014 & 0.049989004 & 0.087013007 \\ \mathrm{H} & -0.643051044 & -2.567791187 & 0.657123047 \\ \mathrm{H} & -2.677916190 & 1.125433080 & -0.174623015 \\ \mathrm{H} & -2.776445200 & -3.685502264 & 0.882785065 \\ \mathrm{H} & -4.824732345 & -0.018886003 & 0.081905007 \\ \mathrm{H} & -4.890391351 & -2.438525177 & 0.606333043 \\ \mathrm{H} & -1.335803098 & 1.872965134 & 1.772920127 \\ \mathrm{H} & 1.437284102 & 1.539844108 & -1.476538105 \\ \mathrm{H} & -0.934377069 & 4.294232311 & 1.843869133 \\ \mathrm{H} & 1.874388134 & 3.918721283 & -1.352469096 \\ \mathrm{H} & 0.685820049 & 5.332578385 & 0.291531023 \\ \mathrm{C} & 1.038790074 & -0.754409056 & -0.103484008 \\ \mathrm{C} & 1.971063140 & -0.973255070 & 1.031330072 \\ \mathrm{O} & 1.660359120 & -0.797655056 & 2.167593156 \\ \mathrm{O} & 3.158603229 & -1.360015100 & 0.633586048 \\ \mathrm{C} & 4.159722297 & -1.532043110 & 1.626242116 \\ \mathrm{H} & 4.327299312 & -0.602421042 & 2.149817156 \\ \mathrm{H} & 5.046691366 & -1.831656129 & 1.092694080 \\ \mathrm{H} & 3.863761278 & -2.296977163 & 2.328879169 \\ \mathrm{C} & 1.417126100 & -1.227859089 & -1.398489102 \\ \mathrm{~N} & 1.701326123 & -1.610865114 & -2.430656173 \\ \mathrm{C} & 1.061695078 & -0.330693023 & 5.469779393 \\ \mathrm{O} & 0.209106015 & 0.275893020 & 4.547322327 \\ \mathrm{H} & 0.486499035 & 0.028256001 & 3.675034265 \\ \mathrm{H} & 1.027795073 & -1.416692100 & 5.407853388 \\ \mathrm{H} & 2.094744150 & -0.013179001 & 5.343039383 \\ \mathrm{H} & 0.736090051 & -0.038293001 & 6.459234462\end{array}$

Coord_9

$\begin{array}{lrrr}C & -1.468397105 & -0.611864046 & 0.246332020 \\ C & -1.542925111 & -1.999901146 & 0.523943035 \\ C & -2.689443195 & 0.084836008 & 0.083907006 \\ C & -2.747135199 & -2.635950191 & 0.654995046\end{array}$




$\begin{array}{lrrr}\text { C } & -3.909238284 & -0.570831039 & 0.216741016 \\ \text { C } & -3.946899283 & -1.932098141 & 0.505962037 \\ \text { C } & -0.000354999 & 1.514719111 & 0.125984011 \\ \text { C } & -0.661485050 & 2.319434165 & 1.067841074 \\ \text { C } & 0.920466068 & 2.130184151 & -0.738969051 \\ \text { C } & -0.422607031 & 3.692559266 & 1.118479083 \\ \text { C } & 1.160790085 & 3.481624251 & -0.677531048 \\ \text { C } & 0.488723035 & 4.278920308 & 0.247605019 \\ \text { C } & -0.198346015 & 0.051380005 & 0.086439008 \\ \text { H } & -0.639416046 & -2.567557185 & 0.652949045 \\ \text { H } & -2.677532193 & 1.125370082 & -0.172346013 \\ \text { H } & -2.771711201 & -3.686957264 & 0.879743063 \\ \text { H } & -4.823349345 & -0.020563002 & 0.085875006 \\ \text { H } & -4.886830353 & -2.440908178 & 0.607727045 \\ \text { H } & -1.333788097 & 1.872430136 & 1.775287126 \\ \text { H } & 1.434740103 & 1.543580110 & -1.478373106 \\ \text { H } & -0.935205067 & 4.294235307 & 1.846280133 \\ \text { H } & 1.869042137 & 3.923089281 & -1.354530099 \\ \text { H } & 0.681045050 & 5.335088384 & 0.291573023 \\ \text { C } & 1.040709077 & -0.751994056 & -0.104557005 \\ \text { C } & 1.971132144 & -0.974118069 & 1.031108072 \\ \text { O } & 1.659405120 & -0.798381056 & 2.167101154 \\ \text { O } & 3.157928227 & -1.364808097 & 0.634953045 \\ \text { C } & 4.156444298 & -1.542073109 & 1.629396118 \\ \text { H } & 4.326730309 & -0.613749045 & 2.154412155 \\ \text { H } & 5.043238362 & -1.844556132 & 1.097168078 \\ \text { H } & 3.856034279 & -2.306653164 & 2.330543167 \\ \text { C } & 1.418917100 & -1.224365090 & -1.399987102 \\ \text { N } & 1.703009124 & -1.605428114 & -2.432928175 \\ \text { C } & 1.062183074 & -0.326176024 & 5.467900395 \\ \text { O } & 0.210310014 & 0.280889019 & 4.545084325 \\ \text { H } & 0.486385034 & 0.030935003 & 3.673025265 \\ \text { H } & 1.023820073 & -1.412224103 & 5.409453387 \\ \text { H } & 2.096239150 & -0.013199998 & 5.338198385 \\ \text { H } & 0.739561052 & -0.029321002 & 6.457014463 \\ & & & \\ \text { Coord_10 } & & \\ \text { C } & -1.445999106 & -0.600720045 & 0.242626017 \\ \text { C } & -1.501793110 & -1.994734143 & 0.493684034 \\ \text { C } & -2.676188194 & 0.081069007 & 0.085920007 \\ \text { C } & -2.697264196 & -2.650615190 & 0.605696041 \\ \text { C } & -3.886974278 & -0.594631045 & 0.199457012 \\ \text { C } & -3.906339281 & -1.961546141 & 0.462933031 \\ \text { C } & -0.008956002 & 1.549124110 & 0.171521010 \\ \text { C } & -0.687307048 & 2.326697165 & 1.123846083 \\ \text { C } & 0.906921067 & 2.193845158 & -0.677176050 \\ \text { C } & -0.469692032 & 3.702176264 & 1.200254086\end{array}$




$\begin{array}{rrrr}\text { C } & 1.126198080 & 3.547521255 & -0.590438041 \\ \text { C } & 0.437092031 & 4.317832310 & 0.344966024 \\ \text { C } & -0.184889011 & 0.083929004 & 0.103899005 \\ \text { H } & -0.590837040 & -2.551537185 & 0.617514045 \\ \text { H } & -2.678021195 & 1.126320082 & -0.150676009 \\ \text { H } & -2.707643193 & -3.705931269 & 0.810596060 \\ \text { H } & -4.808294348 & -0.055280004 & 0.073826005 \\ \text { H } & -4.839264346 & -2.485749177 & 0.549988039 \\ \text { H } & -1.356557096 & 1.856934133 & 1.819346133 \\ \text { H } & 1.433864103 & 1.628380119 & -1.424069101 \\ \text { H } & -0.995242073 & 4.282892308 & 1.935780141 \\ \text { H } & 1.831163132 & 4.011714289 & -1.255554088 \\ \text { H } & 0.612899043 & 5.375881388 & 0.408752028 \\ \text { C } & 1.067088076 & -0.697646049 & -0.092901008 \\ \text { C } & 1.990935142 & -0.930219069 & 1.046003078 \\ \text { O } & 1.668016119 & -0.778814055 & 2.182403155 \\ \text { O } & 3.185043227 & -1.301310092 & 0.652950045 \\ \text { C } & 4.176558299 & -1.487573107 & 1.652826118 \\ \text { H } & 4.332961313 & -0.567709042 & 2.196725158 \\ \text { H } & 5.070953365 & -1.771163126 & 1.122908079 \\ \text { H } & 3.877654281 & -2.268190164 & 2.336745167 \\ \text { C } & 1.460566107 & -1.140656081 & -1.394136100 \\ \text { N } & 1.756868128 & -1.497459106 & -2.432324177 \\ \text { C } & 1.043141075 & -0.368120026 & 5.485412395 \\ \text { O } & 0.189891012 & 0.243802018 & 4.567075328 \\ \text { H } & 0.474082034 & 0.010826003 & 3.692912265 \\ \text { H } & 1.015883071 & -1.453535103 & 5.411053392 \\ \text { H } & 2.074649147 & -0.043085003 & 5.365523389 \\ \text { H } & 0.712603054 & -0.088819004 & 6.477030466\end{array}$

LIIC path connecting the $T_{1}\left({ }^{3} \pi \pi^{*}\right) / S_{0}$ crossing point and $S_{0}$ minimum Coord_1

$\begin{array}{lrrr}C & -1.445999106 & -0.600720045 & 0.242626017 \\ \mathrm{C} & -1.501793110 & -1.994734143 & 0.493684034 \\ \mathrm{C} & -2.676188194 & 0.081069007 & 0.085920007 \\ \mathrm{C} & -2.697264196 & -2.650615190 & 0.605696041 \\ \mathrm{C} & -3.886974278 & -0.594631045 & 0.199457012 \\ \mathrm{C} & -3.906339281 & -1.961546141 & 0.462933031 \\ \mathrm{C} & -0.008956002 & 1.549124110 & 0.171521010 \\ \mathrm{C} & -0.687307048 & 2.326697165 & 1.123846083 \\ \mathrm{C} & 0.906921067 & 2.193845158 & -0.677176050 \\ \mathrm{C} & -0.469692032 & 3.702176264 & 1.200254086 \\ \mathrm{C} & 1.126198080 & 3.547521255 & -0.590438041 \\ \mathrm{C} & 0.437092031 & 4.317832310 & 0.344966024 \\ \mathrm{C} & -0.184889011 & 0.083929004 & 0.103899005 \\ \mathrm{H} & -0.590837040 & -2.551537185 & 0.617514045 \\ \mathrm{H} & -2.678021195 & 1.126320082 & -0.150676009\end{array}$




$\begin{array}{rrrr}\mathrm{H} & -2.707643193 & -3.705931269 & 0.810596060 \\ \mathrm{H} & -4.808294348 & -0.055280004 & 0.073826005 \\ \mathrm{H} & -4.839264346 & -2.485749177 & 0.549988039 \\ \mathrm{H} & -1.356557096 & 1.856934133 & 1.819346133 \\ \mathrm{H} & 1.433864103 & 1.628380119 & -1.424069101 \\ \mathrm{H} & -0.995242073 & 4.282892308 & 1.935780141 \\ \mathrm{H} & 1.831163132 & 4.011714289 & -1.255554088 \\ \mathrm{H} & 0.612899043 & 5.375881388 & 0.408752028 \\ \mathrm{C} & 1.067088076 & -0.697646049 & -0.092901008 \\ \mathrm{C} & 1.990935142 & -0.930219069 & 1.046003078 \\ \mathrm{O} & 1.668016119 & -0.778814055 & 2.182403155 \\ \mathrm{O} & 3.185043227 & -1.301310092 & 0.652950045 \\ \mathrm{C} & 4.176558299 & -1.487573107 & 1.652826118 \\ \mathrm{H} & 4.332961313 & -0.567709042 & 2.196725158 \\ \mathrm{H} & 5.070953365 & -1.771163126 & 1.122908079 \\ \mathrm{H} & 3.877654281 & -2.268190164 & 2.336745167 \\ \mathrm{C} & 1.460566107 & -1.140656081 & -1.394136100 \\ \mathrm{~N} & 1.756868128 & -1.497459106 & -2.432324177 \\ \mathrm{C} & 1.043141075 & -0.368120026 & 5.485412395 \\ \mathrm{O} & 0.189891012 & 0.243802018 & 4.567075328 \\ \mathrm{H} & 0.474082034 & 0.010826003 & 3.692912265 \\ \mathrm{H} & 1.015883071 & -1.453535103 & 5.411053392 \\ \mathrm{H} & 2.074649147 & -0.043085003 & 5.365523389 \\ \mathrm{H} & 0.712603054 & -0.088819004 & 6.477030466\end{array}$

\section{Coord_2}

$\begin{array}{lrrr}\text { C } & -1.449561104 & -0.592380042 & 0.185794016 \\ \text { C } & -1.501404107 & -1.983616145 & 0.463809031 \\ \text { C } & -2.680058194 & 0.081815004 & 0.049620003 \\ \text { C } & -2.698612196 & -2.645711188 & 0.582267041 \\ \text { C } & -3.889549281 & -0.590803041 & 0.184642013 \\ \text { C } & -3.905250282 & -1.960161141 & 0.450504033 \\ \text { C } & -0.001715000 & 1.536446113 & 0.235569015 \\ \text { C } & -0.683534051 & 2.334447167 & 1.162230082 \\ \text { C } & 0.907079064 & 2.172128158 & -0.635771047 \\ \text { C } & -0.471708034 & 3.706442269 & 1.219667086 \\ \text { C } & 1.126547083 & 3.536405257 & -0.568444042 \\ \text { C } & 0.438930033 & 4.310171310 & 0.354639024 \\ \text { C } & -0.162391014 & 0.072446006 & 0.100700007 \\ \text { H } & -0.591277041 & -2.533202183 & 0.613981046 \\ H & -2.684342191 & 1.127897083 & -0.179725014 \\ \text { H } & -2.705567194 & -3.700472265 & 0.791897058 \\ H & -4.812693345 & -0.052800004 & 0.071225003 \\ H & -4.838090350 & -2.482862181 & 0.549827042 \\ H & -1.356678098 & 1.866305132 & 1.852375133 \\ \text { H } & 1.423494102 & 1.608861114 & -1.391341102 \\ H & -0.998000071 & 4.295646310 & 1.945683143\end{array}$




$\begin{array}{lrrr}\text { H } & 1.828588129 & 3.996492288 & -1.241484090 \\ \mathrm{H} & 0.613179047 & 5.370077388 & 0.408264031 \\ \mathrm{C} & 1.053730076 & -0.701290048 & -0.094187004 \\ \mathrm{C} & 2.017558145 & -0.870727063 & 1.018754074 \\ \mathrm{O} & 1.700270124 & -0.707853053 & 2.161883154 \\ \mathrm{O} & 3.211349231 & -1.264613089 & 0.639196046 \\ \mathrm{C} & 4.186475303 & -1.472383106 & 1.647335121 \\ \mathrm{H} & 4.346397313 & -0.554056041 & 2.192674158 \\ \mathrm{H} & 5.081244365 & -1.767235128 & 1.124175083 \\ \mathrm{H} & 3.880540282 & -2.249129162 & 2.332952167 \\ \mathrm{C} & 1.391259101 & -1.255500091 & -1.369576100 \\ \mathrm{~N} & 1.709409120 & -1.581688111 & -2.416642173 \\ \mathrm{C} & 1.043807077 & -0.365385026 & 5.484190397 \\ \mathrm{O} & 0.194936013 & 0.250337019 & 4.563762330 \\ \mathrm{H} & 0.483094033 & 0.018820004 & 3.690173264 \\ \mathrm{H} & 1.016167075 & -1.450596106 & 5.407928388 \\ \mathrm{H} & 2.076016149 & -0.042127002 & 5.367959387 \\ \mathrm{H} & 0.711157050 & -0.089099007 & 6.475945467\end{array}$

\section{Coord_3}

$\begin{array}{lrrr}\text { C } & -1.451603104 & -0.595492043 & 0.098518008 \\ \text { C } & -1.496086108 & -1.975057142 & 0.410330029 \\ \text { C } & -2.680552191 & 0.094452006 & -0.017041000 \\ \text { C } & -2.686040193 & -2.638197188 & 0.551070042 \\ \text { C } & -3.885432282 & -0.581257043 & 0.161832013 \\ \text { C } & -3.889610279 & -1.951381138 & 0.435679032 \\ \text { C } & 0.030176005 & 1.522908108 & 0.320865022 \\ \text { C } & -0.685424050 & 2.328281167 & 1.228648087 \\ \text { C } & 0.915117065 & 2.153131155 & -0.568781038 \\ \text { C } & -0.481929034 & 3.707382267 & 1.248854090 \\ \text { C } & 1.119989079 & 3.513743252 & -0.537665040 \\ \text { C } & 0.427879028 & 4.303269309 & 0.373152025 \\ \text { C } & -0.152674013 & 0.063306003 & 0.118225008 \\ \text { H } & -0.580527043 & -2.503143180 & 0.596052045 \\ \text { H } & -2.696555194 & 1.143363082 & -0.238029017 \\ \text { H } & -2.684536192 & -3.687288264 & 0.784336057 \\ \text { H } & -4.816122345 & -0.049143003 & 0.080739007 \\ \text { H } & -4.819354348 & -2.472952178 & 0.569529041 \\ \text { H } & -1.372924099 & 1.885247137 & 1.923755138 \\ \text { H } & 1.405709101 & 1.568612115 & -1.324737097 \\ \text { H } & -1.015645073 & 4.319169313 & 1.954222142 \\ \text { H } & 1.810530130 & 3.959380283 & -1.230009088 \\ \text { H } & 0.595720044 & 5.364956386 & 0.399370027 \\ \text { C } & 1.043149077 & -0.680784049 & -0.100049008 \\ \text { C } & 2.065354148 & -0.758976057 & 0.982875068 \\ \text { O } & 1.763699129 & -0.575918041 & 2.122886151 \\ \text { O } & 3.239667231 & -1.207431085 & 0.607583045 \\ & & & \end{array}$

S82 


$\begin{array}{lrrr}\mathrm{C} & 4.190368301 & -1.459011104 & 1.637020118 \\ \mathrm{H} & 4.364473315 & -0.561346040 & 2.210422159 \\ \mathrm{H} & 5.091327366 & -1.772397125 & 1.135018084 \\ \mathrm{H} & 3.843886274 & -2.242165163 & 2.298513163 \\ \mathrm{C} & 1.297052094 & -1.393691099 & -1.328405094 \\ \mathrm{~N} & 1.639453116 & -1.729254125 & -2.358455171 \\ \mathrm{C} & 1.044070073 & -0.368275027 & 5.481843396 \\ \mathrm{O} & 0.196836013 & 0.240246016 & 4.554937325 \\ \mathrm{H} & 0.496136035 & 0.015377002 & 3.683329267 \\ \mathrm{H} & 1.014413074 & -1.454065106 & 5.413431387 \\ \mathrm{H} & 2.076608150 & -0.046371003 & 5.365319386 \\ \mathrm{H} & 0.709554051 & -0.084887005 & 6.471105464\end{array}$

\section{Coord_4}

\begin{tabular}{lrrr}
$\mathrm{C}$ & -1.440192105 & -0.599562041 & 0.062085006 \\
$\mathrm{C}$ & -1.508699106 & -1.984299143 & 0.366696026 \\
$\mathrm{C}$ & -2.657600192 & 0.105157008 & -0.081428006 \\
$\mathrm{C}$ & -2.704017197 & -2.626253187 & 0.509124035 \\
$\mathrm{C}$ & -3.873597280 & -0.553999038 & 0.086930005 \\
$\mathrm{C}$ & -3.900966280 & -1.920041139 & 0.382095026 \\
$\mathrm{C}$ & 0.035446001 & 1.508785108 & 0.354142026 \\
$\mathrm{C}$ & -0.680784049 & 2.290439167 & 1.272640094 \\
$\mathrm{C}$ & 0.929883068 & 2.151160155 & -0.513758035 \\
$\mathrm{C}$ & -0.491835036 & 3.671473262 & 1.305226094 \\
$\mathrm{C}$ & 1.115294081 & 3.515166252 & -0.476035037 \\
$\mathrm{C}$ & 0.402317031 & 4.287264307 & 0.431663032 \\
$\mathrm{C}$ & -0.140773012 & 0.049672006 & 0.129695009 \\
$\mathrm{H}$ & -0.604678042 & -2.530116180 & 0.554223039 \\
$\mathrm{H}$ & -2.649775190 & 1.152944085 & -0.309679024 \\
$\mathrm{H}$ & -2.718955198 & -3.670163263 & 0.765705054 \\
$\mathrm{H}$ & -4.794786347 & -0.006901001 & 0.006495000 \\
$\mathrm{H}$ & -4.837106350 & -2.422484174 & 0.537194041 \\
$\mathrm{H}$ & -1.361956096 & 1.829819133 & 1.963128143 \\
$\mathrm{H}$ & 1.444247106 & 1.575540114 & -1.259810091 \\
$\mathrm{H}$ & -1.042485076 & 4.269673306 & 2.007496146 \\
$\mathrm{H}$ & 1.796621129 & 3.974637284 & -1.168920083 \\
$\mathrm{H}$ & 0.534893041 & 5.354027383 & 0.451528034 \\
$\mathrm{C}$ & 1.039713076 & -0.682663052 & -0.066685007 \\
$\mathrm{C}$ & 2.120462154 & -0.626875043 & 0.957962070 \\
$\mathrm{O}$ & 1.895324138 & -0.305067022 & 2.085438150 \\
$\mathrm{O}$ & 3.269720233 & -1.114493081 & 0.559965041 \\
$\mathrm{C}$ & 4.214356303 & -1.408201102 & 1.581607116 \\
$\mathrm{H}$ & 4.423541318 & -0.528831040 & 2.169733154 \\
$\mathrm{H}$ & 5.099624368 & -1.752061124 & 1.073403077 \\
$\mathrm{H}$ & 3.826582274 & -2.186840158 & 2.225059160 \\
$\mathrm{C}$ & 1.216974087 & -1.564943112 & -1.189596086 \\
$\mathrm{~N}$ & 1.439687106 & -2.126363152 & -2.153651156 \\
& & & \\
\hline
\end{tabular}




$\begin{array}{rrrr}\mathrm{C} & 1.044950073 & -0.370922025 & 5.473045395 \\ \mathrm{O} & 0.199553016 & 0.223041014 & 4.534874327 \\ \mathrm{H} & 0.557677042 & 0.073690006 & 3.668782264 \\ \mathrm{H} & 1.027155075 & -1.457792107 & 5.410602390 \\ \mathrm{H} & 2.074597150 & -0.038239004 & 5.364070384 \\ \mathrm{H} & 0.698878048 & -0.085341008 & 6.457717465\end{array}$

\section{Coord_5}

$\begin{array}{lrrr}\mathrm{C} & -1.438255105 & -0.607482046 & 0.049182003 \\ \mathrm{C} & -1.541350110 & -1.992055146 & 0.363970027 \\ \mathrm{C} & -2.642697190 & 0.117365010 & -0.123375009 \\ \mathrm{C} & -2.748575196 & -2.609039189 & 0.484891035 \\ \mathrm{C} & -3.873449280 & -0.518795035 & 0.022643002 \\ \mathrm{C} & -3.932881283 & -1.883030133 & 0.326590026 \\ \mathrm{C} & 0.033209000 & 1.494045109 & 0.354645025 \\ \mathrm{C} & -0.686023052 & 2.249831164 & 1.287350095 \\ \mathrm{C} & 0.922882069 & 2.156126155 & -0.498291036 \\ \mathrm{C} & -0.508796035 & 3.632157261 & 1.347588098 \\ \mathrm{C} & 1.100165078 & 3.520441255 & -0.430335030 \\ \mathrm{C} & 0.379511027 & 4.270132310 & 0.489749035 \\ \mathrm{C} & -0.133452009 & 0.029804004 & 0.129972007 \\ \mathrm{H} & -0.653861045 & -2.560300186 & 0.560835040 \\ \mathrm{H} & -2.611740189 & 1.162517086 & -0.360632025 \\ \mathrm{H} & -2.787706203 & -3.652847260 & 0.740032051 \\ \mathrm{H} & -4.782162342 & 0.043437001 & -0.087761004 \\ \mathrm{H} & -4.880785350 & -2.370153169 & 0.455511035 \\ \mathrm{H} & -1.362026096 & 1.769986125 & 1.969923144 \\ \mathrm{H} & 1.450331104 & 1.597056117 & -1.248577092 \\ \mathrm{H} & -1.063470076 & 4.209159300 & 2.064038148 \\ \mathrm{H} & 1.785906126 & 3.999002287 & -1.106054080 \\ \mathrm{H} & 0.507248038 & 5.336771384 & 0.533718040 \\ \mathrm{C} & 1.040594077 & -0.705789050 & -0.042499003 \\ \mathrm{C} & 2.168090154 & -0.558469041 & 0.928169068 \\ \mathrm{O} & 1.997718142 & -0.133513007 & 2.027794147 \\ \mathrm{O} & 3.289683239 & -1.101156079 & 0.522055037 \\ \mathrm{C} & 4.261737304 & -1.366197097 & 1.527857111 \\ \mathrm{H} & 4.494729321 & -0.470772035 & 2.081725152 \\ \mathrm{H} & 5.129494370 & -1.734362124 & 1.006833071 \\ \mathrm{H} & 3.882565279 & -2.121486154 & 2.203206161 \\ \mathrm{C} & 1.163422085 & -1.728462126 & -1.048208075 \\ \mathrm{~N} & 1.324878096 & -2.429826175 & -1.929947136 \\ \mathrm{C} & 1.048732076 & -0.371180025 & 5.457893394 \\ \mathrm{O} & 0.216449016 & 0.219670017 & 4.505633325 \\ \mathrm{H} & 0.623515042 & 0.140911011 & 3.652229261 \\ \mathrm{H} & 1.046985077 & -1.457851105 & 5.386755388 \\ \mathrm{H} & 2.075551151 & -0.023352999 & 5.374722389 \\ \mathrm{H} & 0.677120047 & -0.098435007 & 6.436921466\end{array}$


Coord_6

$\begin{array}{lrrr}\mathrm{C} & -1.448425103 & -0.623799047 & 0.045570004 \\ \mathrm{C} & -1.609462117 & -2.001944146 & 0.379503025 \\ \mathrm{C} & -2.624256188 & 0.133770008 & -0.196212013 \\ \mathrm{C} & -2.838068203 & -2.576944186 & 0.453335031 \\ \mathrm{C} & -3.878783281 & -0.461979036 & -0.099610008 \\ \mathrm{C} & -3.993831289 & -1.820029132 & 0.222677014 \\ \mathrm{C} & 0.026849004 & 1.459591103 & 0.361746028 \\ \mathrm{C} & -0.691531052 & 2.184082155 & 1.315745095 \\ \mathrm{C} & 0.905342066 & 2.144640157 & -0.479414034 \\ \mathrm{C} & -0.524866037 & 3.566228258 & 1.414550102 \\ \mathrm{C} & 1.073133080 & 3.507866252 & -0.372730028 \\ \mathrm{C} & 0.355189024 & 4.229493307 & 0.572936043 \\ \mathrm{C} & -0.132441011 & -0.012313002 & 0.147256010 \\ \mathrm{H} & -0.752650055 & -2.596092185 & 0.626725048 \\ \mathrm{H} & -2.550108185 & 1.173280086 & -0.447459031 \\ \mathrm{H} & -2.921822208 & -3.614269260 & 0.723766053 \\ \mathrm{H} & -4.763380344 & 0.124603007 & -0.264809022 \\ \mathrm{H} & -4.960788356 & -2.277855163 & 0.310202021 \\ \mathrm{H} & -1.361591096 & 1.680053118 & 1.986975145 \\ \mathrm{H} & 1.437510103 & 1.608490114 & -1.244217090 \\ \mathrm{H} & -1.081917078 & 4.117614298 & 2.149363153 \\ \mathrm{H} & 1.754985125 & 4.011154287 & -1.034647077 \\ \mathrm{H} & 0.478975034 & 5.295291381 & 0.645392045 \\ \mathrm{C} & 1.030470074 & -0.765046054 & 0.018798000 \\ \mathrm{C} & 2.213041158 & -0.529045039 & 0.911758065 \\ \mathrm{O} & 2.112518150 & -0.007746001 & 1.976171145 \\ \mathrm{O} & 3.308056236 & -1.102456077 & 0.477752037 \\ \mathrm{C} & 4.346332315 & -1.291635091 & 1.432894105 \\ \mathrm{H} & 4.613455333 & -0.356832024 & 1.899770137 \\ \mathrm{H} & 5.177977373 & -1.700563124 & 0.883841065 \\ \mathrm{H} & 4.014903291 & -1.993242143 & 2.187271157 \\ \mathrm{C} & 1.119769079 & -1.906532138 & -0.857965059 \\ \mathrm{~N} & 1.245889089 & -2.744870199 & -1.614887115 \\ \mathrm{C} & 1.060362075 & -0.361074026 & 5.422466390 \\ \mathrm{O} & 0.264565018 & 0.246935017 & 4.449606319 \\ \mathrm{H} & 0.725608050 & 0.234128018 & 3.620465259 \\ \mathrm{H} & 1.086518078 & -1.444371103 & 5.313147383 \\ \mathrm{H} & 2.082213148 & 0.010489002 & 5.404901389 \\ \mathrm{H} & 0.633900045 & -0.131424011 & 6.390416459\end{array}$

Coord_7

$\begin{array}{lrrr}C & -1.468928107 & -0.645274049 & 0.050866004 \\ C & -1.717237123 & -2.006589142 & 0.418727030 \\ C & -2.593464185 & 0.155626012 & -0.297194024 \\ C & -2.971411212 & -2.521104181 & 0.420057032\end{array}$

S85 


\begin{tabular}{|c|c|c|c|}
\hline  & 9279 & 028 & 20 \\
\hline C & -4.074117293 & -1.726131127 & \\
\hline $\mathrm{C}$ & 029299004 & 1.401511101 & 0.39712403 \\
\hline C & -0.683351051 & 2.107164152 & $3659440 \Omega$ \\
\hline & 392683066 & 2.096929149 & 4536103 \\
\hline C & 023038 & 7475253 & 237108 \\
\hline $\mathrm{C}$ & 53612075 & 6248 & \\
\hline C & 0.345304025 & 4.164180298 & 0.6484410 \\
\hline C & -0.139869008 & -0.074889006 & 0.19628701 \\
\hline$H$ & -0.909765067 & -2.629440189 & 0.747020054 \\
\hline$\Pi$ & -2.455507178 & 1.183632084 & $-0.56 / 018$ \\
\hline & -3.124599225 & -3.542342255 & 0.720500 \\
\hline & -4.715976339 & 0.231980019 & -0.54063703 \\
\hline & -5.063944364 & -2.141499156 & 0.090261007 \\
\hline & -1.34 & 1.58 & 2.03 \\
\hline & 1.421920102 & 1.573 & -1.22 \\
\hline$\Pi$ & -1.073566079 & 4.022634290 & 2.2371211 \\
\hline & 2123 & 29285 & -0.9795920 \\
\hline & & & \\
\hline 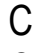 & 1.00 & -0.85 & 0.1 \\
\hline C & 2.242102160 & -0.549053041 & 0.92236106 \\
\hline 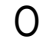 & 2.2008 & 00 & 1.96022 \\
\hline 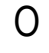 & & & \\
\hline C & 4.44 & -1.1 & 1.36 \\
\hline $\mathrm{H}$ & 4.736740339 & -0.21 & 1.639781 \\
\hline $\mathrm{H}$ & 5.2287 & -1.645 & 0.7128850 \\
\hline 11 & & & \\
\hline C & 1.06 & -2.07 & -0.6 \\
\hline $\mathrm{N}$ & 1.170 & 44217 & -1.30 \\
\hline C & 1.081149078 & -0.332949022 & 5.368229 \\
\hline U & 0.32 & & \\
\hline$\Pi$ & & & \\
\hline & 1.16 & -1.40 & 5.1 \\
\hline$H$ & 2.0841 & 52005 & 5.44495139 \\
\hline & 0.581 & -0.18 & (S) \\
\hline \multicolumn{4}{|c|}{ Coord_8 } \\
\hline$C$ & -1.493 & 177050 & 0.0622270 \\
\hline C & -1.818822131 & -2.008441146 & 0.4648290 \\
\hline C & -2.556240185 & 0.156221009 & -0.398690029 \\
\hline C & & & 0.3997620 \\
\hline C & -3.85 & 06026 & -0.4597130 \\
\hline C & -4.137574298 & -1.650708118 & -0.0622850 \\
\hline C & 0.034891000 & 1.333251098 & 0.437258 \\
\hline C & & & 正 \\
\hline C & & 2.034328148 & -0.4247420 \\
\hline & -0 & 3.41148 & \\
\hline
\end{tabular}

S86 


$\begin{array}{rrrr}\text { C } & 1.035951075 & 3.396351243 & -0.289974019 \\ \text { C } & 0.347889024 & 4.093655295 & 0.694058048 \\ \text { C } & -0.149053012 & -0.152320009 & 0.254251020 \\ \text { H } & -1.060178075 & -2.649146189 & 0.871491063 \\ \text { H } & -2.352107172 & 1.162914086 & -0.710330052 \\ \text { H } & -3.305712240 & -3.477770248 & 0.723198050 \\ \text { H } & -4.651253337 & 0.301875020 & -0.811612059 \\ \text { H } & -5.142494372 & -2.029498146 & -0.105657006 \\ \text { H } & -1.300622093 & 1.503337107 & 2.102468154 \\ \text { H } & 1.395532101 & 1.516436112 & -1.212052088 \\ \text { H } & -1.036046073 & 3.940701284 & 2.311313167 \\ \text { H } & 1.696332124 & 3.920960285 & -0.957707070 \\ \text { H } & 0.469739034 & 5.158256371 & 0.786458057 \\ \mathrm{C} & 0.971681070 & -0.966364071 & 0.234161017 \\ \mathrm{C} & 2.243615162 & -0.622125047 & 0.968349068 \\ \mathrm{O} & 2.248736164 & -0.061259003 & 2.014334143 \\ \mathrm{O} & 3.306059237 & -1.125659081 & 0.394734027 \\ \mathrm{C} & 4.516391324 & -1.101385080 & 1.151096081 \\ \mathrm{H} & 4.812387349 & -0.083080008 & 1.363381096 \\ \mathrm{H} & 5.250147376 & -1.596332113 & 0.535976039 \\ \mathrm{H} & 4.373438313 & -1.640760120 & 2.081915147 \\ \mathrm{C} & 1.001115072 & -2.252146161 & -0.429022030 \\ \mathrm{~N} & 1.087386077 & -3.238201235 & -0.984206071 \\ \mathrm{C} & 1.088595078 & -0.315933021 & 5.337264382 \\ \mathrm{O} & 0.357359027 & 0.353805025 & 4.354671312 \\ \mathrm{H} & 0.877329063 & 0.400208027 & 3.563056258 \\ \mathrm{H} & 1.230687091 & -1.369622096 & 5.097047365 \\ \mathrm{H} & 2.066833151 & 0.135356010 & 5.497128395 \\ \mathrm{H} & 0.532020037 & -0.253120020 & 6.264755452\end{array}$

Coord_9

$\begin{array}{lrrr}\text { C } & -1.523908110 & -0.718446054 & 0.074683007 \\ \text { C } & -1.934776138 & -2.018532143 & 0.504361037 \\ \text { C } & -2.517625179 & 0.139184010 & -0.484210037 \\ \text { C } & -3.221114234 & -2.430920175 & 0.369613025 \\ \text { C } & -3.826542273 & -0.300218023 & -0.630664047 \\ \text { C } & -4.191845303 & -1.588922113 & -0.204886016 \\ \text { C } & 0.040380002 & 1.249525092 & 0.476767037 \\ \text { C } & -0.613411043 & 1.953353138 & 1.481041107 \\ \text { C } & 0.862075063 & 1.936017138 & -0.413406031 \\ \text { C } & -0.433350033 & 3.331302241 & 1.600281114 \\ \text { C } & 1.039457076 & 3.297825239 & -0.283917019 \\ \text { C } & 0.389762026 & 4.001471290 & 0.718298054 \\ \text { C } & -0.172248015 & -0.237802016 & 0.317501021 \\ \text { H } & -1.234517091 & -2.670031190 & 0.988607069 \\ \text { H } & -2.251050161 & 1.127811081 & -0.803424057 \\ \text { H } & -3.503863253 & -3.405341245 & 0.725717050\end{array}$




\begin{tabular}{|c|c|c|c|}
\hline $\mathrm{H}$ & -4.564812328 & 0.351267027 & -1.060870074 \\
\hline $\mathrm{H}$ & -5.205701377 & -1.928262141 & -0.308388022 \\
\hline ト & -1.248263089 & 1.436209105 & 2.176772159 \\
\hline ト & 1.352453099 & 1.409185102 & -1.214092087 \\
\hline & -0.946990067 & 3.868256279 & 2.376661171 \\
\hline & 1.682351124 & 3.816577273 & -0.972947068 \\
\hline & 0.525541039 & 5.065086366 & 0.804544060 \\
\hline & 0.930570067 & -1.074348076 & 0.361256026 \\
\hline C & 2.232746163 & -0.685296047 & 1.015 \\
\hline 0 & 2.284619165 & -0.134332010 & 2.064653147 \\
\hline C & 3.274370235 & -1.111371079 & 0.347675025 \\
\hline C & 4.544155328 & -0.989170071 & 0.983478071 \\
\hline r & 4.796737344 & 0.052301006 & 1.11 \\
\hline ト & 5.246325377 & -1.469739103 & 0.322 \\
\hline 卜 & 4.524666328 & -1.486987107 & 1.94 \\
\hline C & 0.933795069 & -2.412 & -0.18 \\
\hline $\mathrm{N}$ & 1.005349073 & -3.447266249 & -0.645045047 \\
\hline 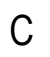 & 1.081278075 & -0.315576022 & \\
\hline 0 & 0.366694026 & 0.361017028 & 4.359305312 \\
\hline ト & 0.904287066 & 0.41 & 3.57 \\
\hline $\mathrm{H}$ & 1.275311090 & -1.352016099 & 5.077790366 \\
\hline ト & 2.032475149 & 0.16 & 5.5671 \\
\hline $\mathrm{H}$ & 0.483298037 & 65024 & 6.2504 \\
\hline \multicolumn{4}{|c|}{ Coord_10 } \\
\hline  & -1.681776120 & -0.640966049 & 0.179419012 \\
\hline C & -2.224746162 & -1.743826126 & 0.832 \\
\hline C & -2.501392180 & 0.133228008 & -0.645971044 \\
\hline C & -3.558730255 & -2.087840149 & 0.651287048 \\
\hline C & -3.830556273 & -0.216633016 & -0.845859061 \\
\hline C & -4.365533315 & -1.330585098 & -0.195822015 \\
\hline C & 0.028662002 & 1.212328086 & 0.461213036 \\
\hline C & -0.630727046 & 2.034235145 & 1.392501101 \\
\hline C & 0.929961069 & 1.786946129 & -0.441234033 \\
\hline C & -0.367966025 & 3.397525244 & 1.419194103 \\
\hline C & 1.176309086 & 3.158129229 & -0.405834028 \\
\hline C & 0.532062038 & 3.954936285 & 0.516519038 \\
\hline C & -0.268981018 & -0.25 & 2030 \\
\hline $\mathrm{H}$ & -1.622261115 & -2.324934169 & 1.503474111 \\
\hline 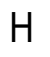 & -2.101863151 & 0.999638070 & -1.138553083 \\
\hline $\mathrm{H}$ & -3.962444283 & -2.936192212 & 1.172639083 \\
\hline 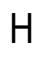 & -4.445592319 & 0.376940030 & -1.496623107 \\
\hline $\mathrm{H}$ & -5.395673389 & -1.599208117 & -0.343334025 \\
\hline $\mathrm{H}$ & -1.314926092 & 1.601517113 & 2.097254149 \\
\hline $\mathrm{H}$ & 1.419948102 & 1.174222085 & -1.176900087 \\
\hline $\mathrm{H}$ & -0.864001061 & 4.023561292 & 2.137513155 \\
\hline & 1.868492137 & 3.591540261 & -1.104577078 \\
\hline
\end{tabular}

S88 


$\begin{array}{lrrr}\mathrm{H} & 0.723771054 & 5.012981362 & 0.536940041 \\ \mathrm{C} & 0.741693054 & -1.154731084 & 0.455984034 \\ \mathrm{C} & 2.130642154 & -0.797395056 & 0.907369063 \\ \mathrm{O} & 2.364699172 & -0.149502013 & 1.876660136 \\ \mathrm{O} & 3.052242222 & -1.331833094 & 0.143662008 \\ \mathrm{C} & 4.403694319 & -1.171757082 & 0.552450041 \\ \mathrm{H} & 4.672502338 & -0.125818008 & 0.555008042 \\ \mathrm{H} & 4.990962360 & -1.712453123 & -0.171699015 \\ \mathrm{H} & 4.546072326 & -1.585268112 & 1.539735108 \\ \mathrm{C} & 0.584022042 & -2.549466181 & 0.116858011 \\ \mathrm{~N} & 0.530946040 & -3.648670263 & -0.160241009 \\ \mathrm{C} & 1.110233078 & -0.338306026 & 5.230317374 \\ \mathrm{O} & 0.458505035 & 0.307693022 & 4.178332302 \\ \mathrm{H} & 1.046195074 & 0.346939024 & 3.433872249 \\ \mathrm{H} & 1.367134101 & -1.366339097 & 4.982752360 \\ \mathrm{H} & 2.019233144 & 0.179870014 & 5.528219400 \\ \mathrm{H} & 0.436779033 & -0.353304026 & 6.076412437\end{array}$

